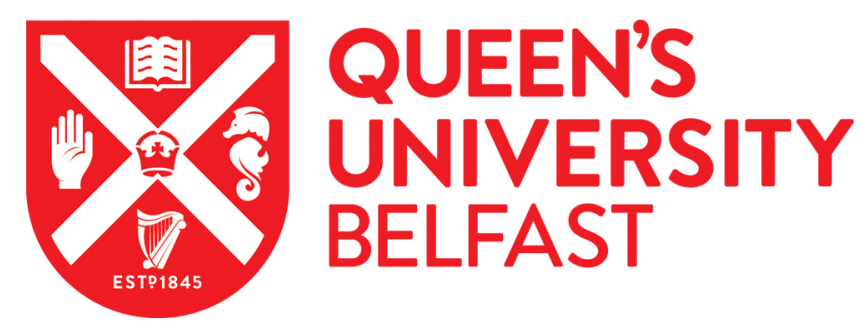

\title{
Roadmap for metal nanoparticles in radiation therapy: current status, translational challenges, and future directions
}

Schuemann, J., Bagley, A., Berbeco, R., Bromma, K., Butterworth, K. T., Byrne, H., Chithrani, D. B., Cho, S. H., Cook, J. R., Favaudon, V., Gholami, Y. H., Gargioni, E., Hainfeld, J. F., Hespeels, F., Heuskin, A-C., Ibeh, U. M., Kuncic, Z., Kunjachan, S., Lacombe, S., ... Krishnan, S. (2020). Roadmap for metal nanoparticles in radiation therapy: current status, translational challenges, and future directions. Physics in Medicine and Biology. https://doi.org/10.1088/1361-6560/ab9159

Published in:

Physics in Medicine and Biology

Document Version:

Peer reviewed version

Queen's University Belfast - Research Portal:

Link to publication record in Queen's University Belfast Research Portal

\section{Publisher rights}

(C) 2020 Institute of Physics and Engineering in Medicine. This manuscript is distributed under a Creative Commons Attribution-

NonCommercial-NoDerivs License (https://creativecommons.org/licenses/by-nc-nd/4.0/), which permits distribution and reproduction for noncommercial purposes, provided the author and source are cited.

\section{General rights}

Copyright for the publications made accessible via the Queen's University Belfast Research Portal is retained by the author(s) and / or other copyright owners and it is a condition of accessing these publications that users recognise and abide by the legal requirements associated with these rights.

Take down policy

The Research Portal is Queen's institutional repository that provides access to Queen's research output. Every effort has been made to ensure that content in the Research Portal does not infringe any person's rights, or applicable UK laws. If you discover content in the Research Portal that you believe breaches copyright or violates any law, please contact openaccess@qub.ac.uk. 
ACCEPTED MANUSCRIPT

\section{Roadmap for metal nanoparticles in radiation therapy: current status, translational challenges, and future directions}

To cite this article before publication: Jan Schuemann et al 2020 Phys. Med. Biol. in press https://doi.org/10.1088/1361-6560/ab9159

\section{Manuscript version: Accepted Manuscript}

Accepted Manuscript is "the version of the article accepted for publication including all changes made as a result of the peer review process, and which may also include the addition to the article by IOP Publishing of a header, an article ID, a cover sheet and/or an 'Accepted

Manuscript' watermark, but excluding any other editing, typesetting or other changes made by IOP Publishing and/or its licensors"

This Accepted Manuscript is $\odot 2020$ Institute of Physics and Engineering in Medicine.

During the embargo period (the 12 month period from the publication of the Version of Record of this article), the Accepted Manuscript is fully protected by copyright and cannot be reused or reposted elsewhere.

As the Version of Record of this article is going to be / has been published on a subscription basis, this Accepted Manuscript is available for reuse under a CC BY-NC-ND 3.0 licence after the 12 month embargo period.

After the embargo period, everyone is permitted to use copy and redistribute this article for non-commercial purposes only, provided that they adhere to all the terms of the licence https://creativecommons.org/licences/by-nc-nd/3.0

Although reasonable endeavours have been taken to obtain all necessary permissions from third parties to include their copyrighted content within this article, their full citation and copyright line may not be present in this Accepted Manuscript version. Before using any content from this article, please refer to the Version of Record on IOPscience once published for full citation and copyright details, as permissions will likely be required. All third party content is fully copyright protected, unless specifically stated otherwise in the figure caption in the Version of Record.

View the article online for updates and enhancements. 


\section{Roadmap for Metal Nanoparticles in Radiation}

\section{Therapy: Current Status, Translational}

\section{Challenges, and Future Directions.}

Jan Schuemann ${ }^{1}$, Alexander F. Bagley², Ross Berbeco ${ }^{3}$, Kyle Bromma ${ }^{4}$, Karl T. Butterworth ${ }^{5}, \mathrm{H}$. Byrne $^{6}$, B. Devika Chithrani ${ }^{4}$, Sang Hyun Cho ${ }^{7}$, Jason R. Cook $^{8}$, Vincent Favaudon ${ }^{9}$, Y. Gholami ${ }^{6}$, Elisabetta Gargioni ${ }^{10}$, James F. Hainfeld ${ }^{11}$, Félicien Hespeels ${ }^{12}$, Anne-Catherine Heuskin ${ }^{12}$, Udoka M. Ibeh ${ }^{3}$, Z. Kuncic ${ }^{6,13}$, Sijumon Kunjachan ${ }^{3}$, Sandrine Lacombe ${ }^{14}$, Stéphane Lucas ${ }^{12}$ François Lux ${ }^{15,16}$, Stephen McMahon ${ }^{5}$, Dmitry Nevozhay ${ }^{17,18}$, Wilfred Ngwa ${ }^{3}$, J. Donald Payne ${ }^{19}$, Sébastien Penninckx ${ }^{12}$, Erika Porcel ${ }^{14}$, Kevin M. Prise ${ }^{5}$, Hans Rabus $^{20}$, Sharif M. Ridwan ${ }^{21}$, Benedikt Rudek $^{1,22}$, Léon Sanche ${ }^{23}$, Bijay Singh ${ }^{24}$, Henry M. Smilowitz ${ }^{21}$, Konstantin V. Sokolov ${ }^{17,25,26}$, Srinivas Sridhar ${ }^{24}$, Yaroslav Stanishevskiy ${ }^{11}$, Wonmo Sung ${ }^{1}$, Olivier Tillement ${ }^{15,16}$, Needa Virani ${ }^{3}$, Wassana Yantasee ${ }^{27}$ and Sunil Krishnan ${ }^{28}$

1 Department of Radiation Oncology, Massachusetts General Hospital \& Harvard Medical School, Boston, MA 02114, USA

2 Department of Radiation Oncology, The University of Texas MD Anderson Cancer Center, Houston, TX

3 Department of Radiation Oncology, Brigham and Women's Hospital, Dana-Farber Cancer Institute and Harvard Medical School, Boston, MA USA

4 Department of Physics and Astronomy, University of Victoria, Victoria, BC, Canada;

5 Centre for Cancer Research and Cell Biology, Queen's University Belfast, Belfast, Northern Ireland, United Kingdom.

6 The University of Sydney, Faculty of Science, Institute of Medical Physics, School of Physics, Australia

7 Department of Radiation Physics \& Department of Imaging Physics, The University of Texas MD Anderson Cancer Center, Houston, TX 77030, USA

8 NanoHybrids, Inc., Austin, TX 78744, United States of America

9 Institut Curie, Inserm U 1020-CNRS UMR 3347, University Paris-Saclay, University Paris Sud, 91405 Orsay Cedex, France

10 Department of Radiotherapy and Radiation Oncology, University medical center Hamburg-Eppendorf, 20246 Hamburg, Germany

11 Nanoporbes, Yaphank, NY, USA

12 NAmur Research Institute for LIfe Sciences (NARILIS), Laboratory of Analysis by Nuclear Reactions, University of Namur, 61 rue de Bruxelles, 5000 Namur, Belgium.

13 The University of Sydney Nano Institute, Australia

14 ISMO (UMR 8625), University Paris-Saclay, University Paris Sud, CNRS, 91405 Orsay Cedex, France

15 NH TherAguix SAS, Villeurbanne, France

16 Univ Lyon Université Claude Bernard Lyon 1, CNRS, Institut Lumière Matière, LYON, France 


\begin{abstract}
:
This roadmap outlines the potential roles of metallic nanoparticles (MNPs) in the field of radiation therapy. MNPs made up of a wide range of materials (from Titanium, $Z=22$, to Bismuth, $Z=83$ ) and a similarly wide spectrum of potential clinical applications, including diagnostic, therapeutic (radiation dose enhancers, hyperthermia inducers, drug delivery vehicles, vaccine adjuvants, photosensitizers, enhancers of immunotherapy) and theranostic (combining both diagnostic and therapeutic), are being fabricated and evaluated. This roadmap covers contributions from experts in these topics summarizing their view of the current status and challenges, as well as expected advancements in technology to address these challenges.
\end{abstract}

\title{
Contents:
}

List of contributions:

\section{Overview of metal nanoparticles for Imaging and Therapy}

1. Gold Nanoparticles for Imàging and Therapy - James F. Hainfeld

2. Magnetic Nanoparticles for MRI and Radiotherapy - Hilary Byrne, Yaser Gholami and Zdenka Kuncic

3. Gadolinium Nahoparticles for Imaging and Therapy in Oncology - François Lux, Ross Berbeco, Olivier Tillement

4. Iodine Nanoparticles - James F. Hainfeld, Sharif M. Ridwan, Yaroslav Stanishevskiy, Henry M. MNP Imaging

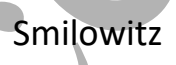

5. Optical and Photoacoustic Imaging of GNPs - Dmitry Nevozhay and Konstantin V. Sokolov 
6. In Vivo Imaging of Metallic Nanoparticles Using Benchtop X-ray Fluorescence Computed Tomography Techniques - Sang Hyun Cho

\section{Modeling, Measuring and Mechanisms of NPs for Therapy}

7. Micro- and Nanodosimetry of MNPs - Hans Rabus, Elisabetta Gargioni

8. Can Physics Explain the Radiation Enhancement Effects of MNPs? - Anne-Catherine Heuskin, Sébastien Penninckx, Félicien Hespeels, Stéphane Lucas

9. Role of Low Energy Electrons in Local MNP Radiation Enhancement- Léon Sanche

10. Modeling of MNP Effects with Monte Carlo - Wonmo Sung and Benedikt Rudek

11. Chemical Effects in NPs Induced Radio-Enhancement - Sandrine Lacombe, Vincent Favaudon, Erika Porcel

12. Modelling the Biological Effects of Metal Nanoparticles - Stephen McMahon

13. Molecular Mechanisms of Metal Nanoparticle (MNP)-Mediated Radiation Enhancement - Karl T. Butterworth and Kevin M. Prise

\section{MNPs for Therapy}

14. Optimization of GNP-based Cancer Therapeutics - B. Devika Chithrani

15. Targeting Tumor Vasculature with Nanoparticles for Radiation Therapy - Needa Virani, Ross Berbeco, Sijumon Kunjachan

\section{MNP Translation \& Theranostics}

16. Clinical Translation of Gold Nanoparticles for In-Vivo Use - Jason R. Cook and J. Donald Payne

17. Next-Generation Metallic Nanoparticles for Cancer Radiation Therapy - Bijay Singh, Srinivas Sridhar

18. Theranostics Metallic Nanoparticles - Udoka M. Ibeh, Wilfred Ngwa

19. Metallic Nanoparticles for Enhancing Radiotherapy and Immunotherapy - Alexander F. Bagley, Wassana Yantasee, Sunil Krishnan 


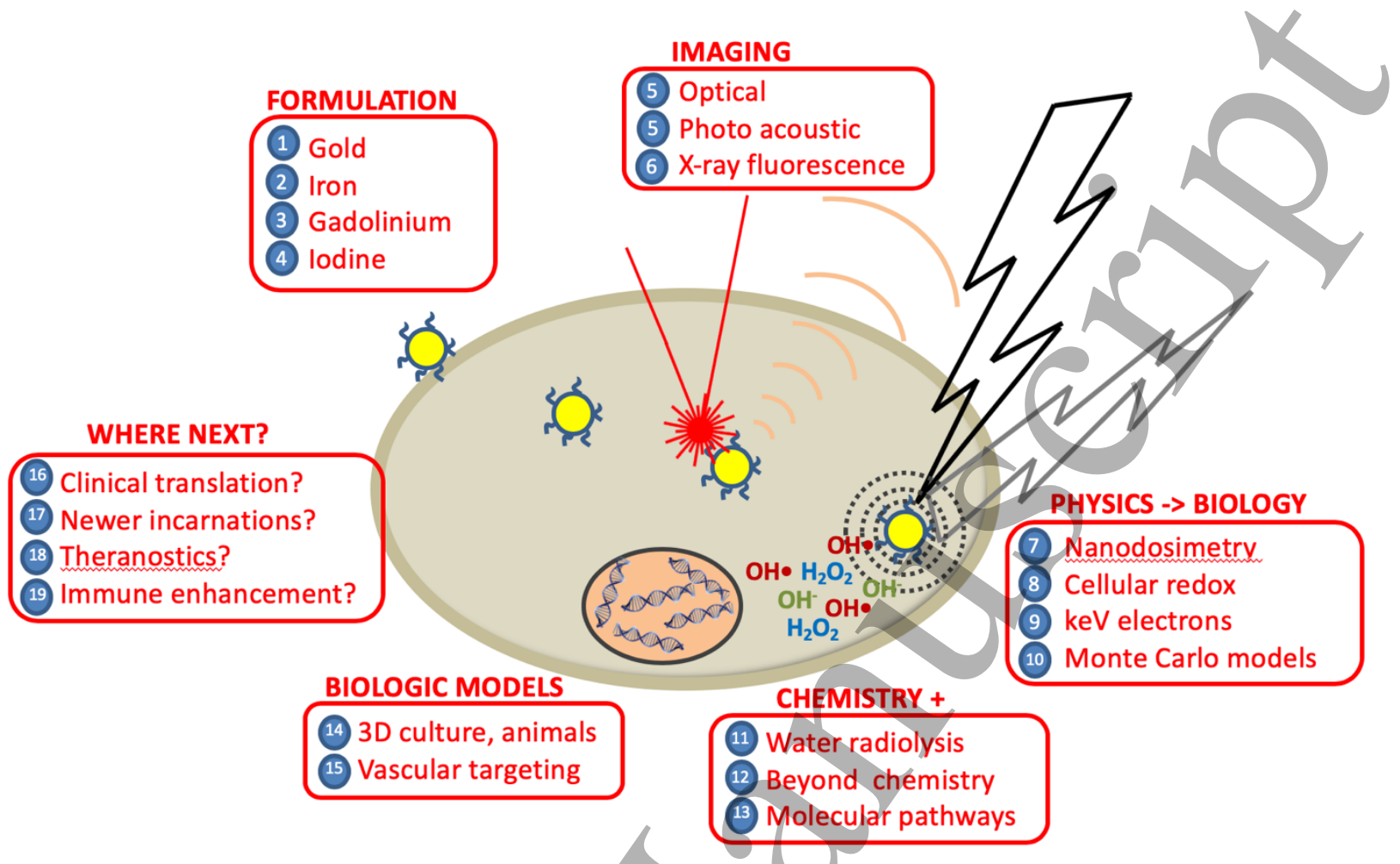

Figure 1: Graphic illustration of how each contribution in this collection connects to the study of (metallic) nanoparticles and their interaction with cells and radiation.

\section{Introduction:}

MNPs are characterized by their small size (typically $<100 \mathrm{~nm}$ ), large surface area to volume ratio, and high interaction cross-section with incident ionizing or non-ionizing radiation. The high radiation interaction cross-section is well known but modeling, mechanistic underpinning, and extensive characterization of consequences of physical, chemical and biological interplay in mediating anti-tumor effects remains inadequately understood. A variety of MNPs are also quite readily endowed with biological functionalities such as stealth coatings that help them evade capture and clearance by circulating and tissue-resident macrophages. MNPs can further be decorated with tumor targeting ligands that facilitate tumor homing or coupled with photosensitizers or other biological mediators of tissue damage. To penetrate fortified stromal barriers erected by tumors including vasculature, fibrous/connective tissue, and immune evasion strategies, MNPs can further be armed with multiple ligands specific for each barrier. Not surprisingly, this interface between nanomaterial design, radiation interaction, and tumor biology is a fertile ground for active research by multiple disciplines, often with non-intersecting boundaries.

This special issue roadmap is an attempt to collate and weave together the extant knowledge in the arena of nanoparticle research. By including experts from diagnostic, therapeutic and theranostic 
1

2

3

4

5

6

7

8

9

10

11

12

13

14

15

16

17

18

applications of MNPs, we endeavor to provide a broad overview in the field, benchmarking what is known today, which gaps in knowledge and technology will be addressed in the near future and forecasting what the future horizons hold in store. 


\title{
Gold Nanoparticles for Imaging and Therapy
}

\author{
James F. Hainfeld \\ Nanoprobes, Yaphank, NY, USA
}

\section{Status}

Imaging: The extensive historical wealth of chemistry of gold nanoparticles (GNPs) with their high density, high atomic number, variable size, and facile attachment of a variety of ligands make them ideal as imaging agents. Once passivated to be biologically tolerated, they can be injected intravenously or locally, or embedded in other materials and devices. Imaging can be by X-ray absorption (e.g., CT or microCT), but newer devices rely on X-ray fluorescence(Manohar et al 2016); gold can also be radioactive. The nanoparticle aspect gives them more flexibility and control by adjusting size, shape, and adding one or more targeting ligands(Panahi et al 2017). This allows control over blood half-life, clearance, and specific uptake. Results are showing excellent vasculature imaging as well as tumor imaging(Hainfeld et al 2006) and other targets such as stroke clots(Kim et al 2017). Cells (e.g., macrophages) have been loaded with GNPs and shown to target vulnerable plaque(Chhour et al 2016). Gold nanoparticles have been the favorite choice for electron microscope studies for cell and molecular labeling (e.g., with immunogold). GNPs have been combined with fluorescent molecules to provide dual labeling(Takizawa and Robinson 2003). Some GNPs are fluorescent. GNPs are also useful for photoacoustic imaging and as SERS probes. Silver and gold enhancement, which catalytically deposits silver or gold on the GNPs grows their size to a level detectable by the light microscope for histology or other studies(Hayat 1995). This metallic growth also increases the sensitivity. Larger GNPs ( 30nm) can be seen in low (pM) concentration by the naked eye and are commonly used in lateral flow devices, such as the pregnancy test kit, or strips to detect infectious diseases. In short, GNPs have been and are a tremendously useful platform for imaging.

Therapy: One group showed cells grown on a gold monolayer were killed 160 times more than those grown on plastic after X-ray irradiation(Regulla et al 1998). X-rays are absorbed by gold atoms, knocking out electrons that create ionizations and free radicals, so more dose is deposited locally around the gold. It was shown that gold nanoparticles delivered to tumors (in test animals) and irradiated resulted in much greater tumor destruction than radiation alone(Hainfeld et al 2004). A number of groups have contributed extensive theoretical modeling to better understand and predict these effects ${ }^{10}$ (Her et al 2017). Gold better absorbs kV energy X-rays ( $<400 \mathrm{keV}$ ) than MV ( $\left.>4 \mathrm{MV}\right) \mathrm{X}$-rays 


\section{Current and future challenges}

For ex-vivo or animal imaging at the macroscopic, light, and electron microscope levels, new targeting moieties and reactive groups will further add functionality and enable new applications. The challenge will be to synthesize these functionalized nanoparticles and yet retain appropriate stability. For example, putting a novel targeting moiety on the GNP surface may induce unwanted aggregation.

In vivo imaging and therapy with GNPs has been achieved for small animals. However, translation to human use has lagged and largely not been done due to several problems: 1) poor clearance, 2) skin discoloration at high levels, 3) possibly high cost (although reasonable if benefit is great). CT imaging has high resolution but low sensitivity so relatively large amounts of GNPs need to be injected, raising the issue of toxicity. For therapy, only a small amount of what is injected iv ends up in the tumor, again requiring relatively large amounts to be injected. Local injections mostly avoid this problem but do not adequately address most clinical cases where there are multiple lesions, some microscopic, and a direct injection may not fully cover all cells, leaving some undertreated with resulting recurrence(Smilowitz et al 2018). Clearance is also a problem. Less than $5 \mathrm{~nm}$ particles can exit rapidly via the kidneys. However, this fast clearance does not give enough time to maximally load tumors or other targets. IV antibodies take 24 hours and multi-passes to maximally load targets. These particles then clear via the hepatobiliary route, but unfortunately, macrophages take up the GNPs throughout the body and because gold is insoluble and not digestible it just stays in cells. Liver clearance has been reported to be only $9 \%$ in 6 months(Sadauskas et al 2009). This whole body retention will be a problem for FDA approval. Because GNPs are highly colored, cells in the skin (probably mostly macrophages) take them up and skin discoloration is virtually permanent. Gold is also costly. Unless small amounts 


\section{Advances in science and technology to meet challenges}

For tumor and other targeted imaging and therapy of GNPs in humans, one solution is to use small acceptable amounts of gold. Imaging using fluorescent photons is more sensitive than $\mathrm{CT}$, and development of this equipment is underway. Heating of superficial or deeper tissue with light pipes and NIR appears to work well with small amounts of gold nanorods or nanoshells injected iv. For better clearance $<6 \mathrm{~nm}$ particles in a biodegradable matrix have been developed to extend blood half-life, then later break down and be cleared through the kidneys. However, during the long blood residence they will be taken up by macrophages and may have difficulty exiting the lysosomes, even when the polymer is digested. Because of the ease of chemically functionalizing GNPs with thiols that bind to their surface the problems of passivating them to avoid toxicity and linking targeting molecules (e.g., peptides and antibodies) does not appear to be a problem for GNPs as it can be for other types of NPs. The amount of gold needed for X-ray dose enhancement (at kV) is calculated to be rather high ( 2\% Au by weight) for a significant effect. However, some experiments have shown good dose enhancement with far less gold and using MV photons(Wolfe et al 2015). Usually a poorly understood biological effect is invoked to explain such unexpected results, but indicates that some GNPs might be acceptable for clinical use. Other high atomic number nanoparticles (e.g., Gd, Ho, Ti, I) may provide favorable alternatives.

Concerning the kV vs. MV controversy, kV methods are being revisited: 1) Grid, microbeam, and minibeams. Spatially segmented beams (intervening non irradiated zones) were found to eliminate the high skin entrance dose of kV X-rays(Dilmanian et al 2019); 2) Stereotactic kV irradiation also avoids entrance dose; 3) CT machines use bow-tie filters that upon rotation produce isodose in the subject, again avoiding skin entrance dose and dose fall-off(Schultz et al 2011). kV machines are much less expensive, portable, and require less shielding, and could make nanoparticle dose enhancement cancer treatments available to developing countries.

\section{Concluding remarks}




\section{References:}

GNPs have incredibly useful properties and flexibility making them one of the best choices for imaging all the way from EM to animal imaging, using various methods including electrons, ultrasound, protons and X-rays. For therapy a number of forms have been developed for heating, drug release, and dose enhancement.

I have been making and using GNPs for over 40 years. A focus has been their dose enhancement for improved cancer therapy. They are among the best radioenhancers ever found. However, the problems with clinical translation have driven me to develop iodine nanoparticles that have better clearance, are colorless (no skin discoloration) and are less expensive(Hainfeld et al 2019, 2018). It is hoped that these, GNPs, or other dose enhancing NPs will soon be useful for patients.

Ahmad R, Fu J, He N and Li S 2016 Advanced Gold Nanomaterials for Photothermal Therapy of Cancer. J Nanosci Nanotechnol 16 67-80

Butterworth K T, McMahon S J, Currell F J and Prise K M 2012 Physical basis and biological mechanisms of gold nanoparticle radiosensitization Nanoscale 44830

Chhour P, Naha P C, O'Neill S M, Litt H I, Reilly M P, Ferrari VA and Cormode D P 2016 Labeling monocytes with gold nanoparticles to track their recruitment in atherosclerosis with computed tomography. Biomaterials 87 93-103

Cui L, Her S, Borst G R, Bristow R G, Jaffray D A and Allen C 2017 Radiosensitization by gold nanoparticles: Will they ever make it to the clinic? Radiother Oncol 124 344-56

Dilmanian F A, Krishnan S, McLaughlin W E, Lukaniec B, Baker J T, Ailawadi S, Hirsch K N, Cattell R F, Roy R, Helfer J, Kruger K, Spuhler K, He Y, Tailor R, Vassantachart A, Heaney D C, Zanzonico P, Gobbert M K, Graf J S, Nassimi J R, Fatemi N N, Schweitzer M E, Bangiyev L and Eley J G 2019 Merging Orthovoltage X-Ray Minibeams spare the proximal tissues while producing a solid beam at the target. Sci Rep 91198

Hainfeld J F, Ridwan S M, Stanishevskiy Y, Panchal R, Slatkin D N and Smilowitz H M 2019 lodine nanoparticles enhance radiotherapy of intracerebral human glioma in mice and increase efficacy of chemotherapy. Sci Rep $\mathbf{9} 4505$

Hainfeld J F, Ridwan S M, Stanishevskiy Y, Smilowitz N R, Davis J and Smilowitz H M 2018 Small, Long Blood Half-Life lodine Nanoparticle for Vascular and Tumor Imaging. Sci Rep 813803

Hainfeld J F, Slatkin D N and Smilowitz H M 2004 The use of gold nanoparticles to enhance radiotherapy in mice. Phys. Med. Biol. 49 N309-15

Hainfeld J F, Slatkin D N, Focella T M and Smilowitz H M 2006 Gold nanoparticles: a new X-ray contrast agent. Br J Radiol 79 248-53 
Hayat M A 1995 Immunogold-Silver Staining (CRC Press)

Her S, Jaffray D A and Allen C 2017 Gold nanoparticles for applications in cancer radiotherapy: Mechanisms and recent advancements Advanced Drug Delivery Reviews 109 84-101

Kim D-E, Kim J-Y, Schellingerhout D, Ryu J H, Lee S-K, Jeon S, Lee J S, Kim J, Jang H J, Park J E, Kim E J, Kwon I C, Ahn C-H, Nahrendorf M and Kim K 2017 Quantitative Imaging of Cerebral Thromboemboli In Vivo: The Effects of Tissue-Type Plasminogen Activator. Stroke 48 1376-85

Manohar N, Reynoso F J, Diagaradjane P, Krishnan S and Cho S H 2016 Quantitative imaging of gold nanoparticle distribution in a tumor-bearing mouse using benchtop $x$-ray fluorescence computed tomography. Sci Rep 622079

Panahi Y, Mohammadhosseini M, Nejati-Koshki K, Abadi A J N, Moafi H F, Akbarzadeh A and Farshbaf M 2017 Preparation, Surface Properties, and Therapeutic Applications of Gold Nanoparticles in Biomedicine. Drug Res (Stuttg) 67 77-87

Regulla D F, Hieber L B and Seidenbusch M 1998 Physical and biological interface dose effects in tissue due to X-ray-induced release of secondary radiation from metallic gold surfaces. Radiat. Res. 150 92-100

Sadauskas E, Danscher G, Stoltenberg M, Vogel U, Larsen A and Wallin H 2009 Protracted elimination of gold nanoparticles from mouse liver. Nanomedicine 5 162-9

Schuemann J, Berbeco R, Chithrani D B, Cho SH, Kumar R, McMahon S J, Sridhar S and Krishnan S 2016 Roadmap to Clinical Use of Gold Nanoparticles for Radiation Sensitization. Int. J. Radiat. Oncol. Biol. Phys. 94 189-205

Schultz B-J, Wust P, Lüdemann L, Jost G and Pietsch H 2011 Monte Carlo simulation of contrastenhanced whole brain radiotherapy on a CT scanner. Med. Phys. 38 4672-80

Smilowitz H M, Meyers A, Rahman K, Dyment N A, Sasso D, Xue C, Oliver D L, Lichtler A, Deng X, Ridwan S M, Tarmu L J, Wu Q, Salner A L, Bulsara K R, Slatkin D N and Hainfeld J F 2018 Intravenouslyinjected gold nanoparticles (AuNPs) access intracerebral F98 rat gliomas better than AuNPs infused directly into the tumor site by convection enhanced delivery. Int I Nanomedicine 13 3937-48

Takizawa T and Robinson J M 2003 Correlative microscopy of ultrathin cryosections is a powerful tool for placental research. Placenta 24 557-65

Wolfe T, Chatterjee D, Lee J, Grant J D, Bhattarai S, Tailor R, Goodrich G, Nicolucci P and Krishnan S 2015 Targeted gold nanoparticles enhance sensitization of prostate tumors to megavoltage radiation therapy in vivo. Nanomedicine 11 1277-83 


\title{
Magnetic Nanoparticles for MRI and Radiotherapy
}

\author{
H. Byrne ${ }^{1}$, Y. Gholami ${ }^{1}$ and Z. Kuncic ${ }^{1,2}$ \\ ${ }^{1}$ The University of Sydney, Faculty of Science, Institute of Medical Physics, School of Physics, Australia \\ 2 The University of Sydney Nano Institute, Australia
}

\section{Status}

In addition to demonstrating therapeutic gain with nanoparticle-enhanced radiotherapy, it is also advantageous to incorporate imaging to aid in diagnosis, monitor treatment response or to track nanoparticle uptake. Many options exist for leveraging existing diagnostic procedures (CT contrast, magnetic resonance imaging (MRI) contrast, positron emission tomography (PET) tracers). MRI stands out due to providing soft tissue contrast and use of non-ionizing radiation. Nanoparticles enhancing MRI contrast can therefore show detailed organ-specific uptake and can exploit differential tissue uptake in disease diagnosis without exposing patients to extra dose.

Current clinically-used MRI contrast agents contain gadolinium (Gd) which, with seven unpaired electrons, confers strong longitudinal (T1) contrast. Chelated Gd nano-structures can provide diagnostic-quality MRI contrast. However, MRI contrast can also be achieved with $\mathrm{Fe} 3+, \mathrm{Fe} 2+, \mathrm{Mn2}+$ and super-paramagnetic iron oxide nanoparticles (SPIONs).

SPIONs are well known for their MRI contrast-enhancing capability, due primarily to their ability to shorten the transverse (T2) relaxation time of water protons (Weinstein et al. 2010). The current status of SPION-enhanced contrast MRI is evolving rapidly as concern grows over long-term toxicity of conventional Gd-based contrast agents (Fornell 2018).

In radiotherapy, enhancement by SPIONs has been investigated in simulation studies (McMahon et al. 2016) and demonstrated in vivo using synchrotron x-rays (Choi et al. 2012) and proton beams (Kim et al. 2012). Remarkably, none of these studies explored the potential to leverage the MRI contrast-enhancing properties of SPIONs alongside their radio-enhancement properties. Such studies are needed, given the increasing role of MRI in treatment planning and the urgent need for highprecision measurement of the Bragg peak in particle therapy.

\section{Current and future challenges}

In addition to addressing the challenges in clinical translation, a novel nanotheranostic for radiotherapy enhancement must be forward-looking, taking advantage of and enhancing emerging technologies. The combination of imaging with therapy is an overarching theme of these emerging techniques, with utilisation of MRI in particular expected to increase in future. Many nanoparticles for 
radio-enhancement are currently under development or have been reported on over the last 20 years, but none have yet been successfully translated for clinical use (Kuncic and Lacombe 2018; Schuemann et al. 2016).

Three recent innovations invite the development of magnetic nanoparticles for MRI and radiotherapy which can rise to this challenge:

\section{The MRI-linac}

MRI-linac technology enables image-guided radiotherapy with precise targeting of soft-tissue structures (Liney et al. 2018). Best practice implementation and workflows exploiting the advantages of time-of-treatment MR imaging are still being explored (Liney et al. 2019). This provides an opportunity for magnetic nanoparticle enhancement to be incorporated as part of standardised treatment as that is developed. Along with visualisation of anatomical changes during treatment, the MRI-linac offers the tantalizing prospect of observing the effects and perhaps effectiveness of radiation treatment inter- and intra-fraction. This poses a challenge for nanoparticle treatments to become adaptive, identifying and responding to individual patient response during a course of treatment.

\section{Particle therapy}

In particle therapy, a key current challenge to be met is integration of image-guidance techniques, similar to that successfully used in conventional MV-beam radiotherapy. MRI offers arguably the best option, especially with pencil beam scanning, where a relatively straightforward technique can be applied to correctly locate the Bragg peak for each pencil beam (Oborn et al. 2017). A future challenge is leveraging SPIONs for enhancing both MRI contrast and radiotherapeutic efficacy in particle therapy (Liu et al. 2018). Feasibility studies are required to explore the potential of this concept.

\section{Targeted internal radiotherapy}

To date, the effects of radio-enhancement in many in vivo, in vitro and in silico studies have mainly been considered in the context of radiation delivered by an external beam (i.e. external beam radiotherapy or brachytherapy) (Schuemann et al. 2016). Targeted internal radionuclide therapy is an alternative treatment approach to achieve more localized radiotherapy by delivering short range radiation via internalization of radioisotope in a tumour (Zhang et al. 2010). Leveraging an appropriate nanoparticle delivery vehicle gives access to MRI-based tracking and diagnostics (Gholami et al. 2015).

\section{Advances in science and technology to meet challenges}

1. Gd-based nanoparticles for MRI-linac application 
Radio-enhancing nanoparticles with inbuilt MRI contrast are well within current capabilities, and perfectly suited to take advantage of the recent development of MRI-linacs. Utilising conventional Gd contrast, the $\mathrm{AGuIX}^{\circledR}$ nanoparticle has been designed for radio-enhancement and MRI contrast. This nanotheranostic has undergone extensive pre-clinical evaluation and has progressed to phase I clinical trial using conventional separate MRI and radiotherapy treatment (Verry et al. 2019). Its applicability to combined MRI-linac systems is obvious, where time-of-treatment imaging gives information on nanoparticle uptake at each treatment fraction.

\section{SPIONs for MRI-guided particle therapy}

To demonstrate the feasibility of SPION radio-enhancement in particle therapy, advances are needed in both simulation and experimental approaches. In simulations, more studies are required to advance our knowledge of the physico-chemical stage of radiation damage and to correctly quantify the radiochemistry processes in the immediate vicinity of activated SPIONs (Rudek et al. 2019). Technological advances are also needed in both software and hardware to enable MRI-guidance in particle therapy (Oborn et al. 2017), which will allow new experiments to be designed to evaluate radiotherapeutic gain by SPIONs.

\section{Radio-labelled SPIONs for targeted internal radiotherapy and PET/MRI}

Nanoparticles can be labelled with various radioisotopes for use in both internal radionuclide therapy and diagnostic imaging (Boros et al. 2015; Yuan et al. 2018). SPIONs are the best candidates for a radiolabelled nano-theranostic platform (Figure 1) due to their favourable physical and chemical properties for chelate-free radiolabelling (Yuan et al. 2018), their MRI contrast properties, and their excellent biocompatibility (Iv et al. 2015).

The six main types of radiation clinically used for internal radionuclide therapy and emission tomography are: $\beta^{-}, \beta^{+}, \alpha$ particles, Auger electrons, conversion electrons, and $\gamma$-rays (Ersahin et al. 2011). As the energy of the emitted particles is typically in the kilo-electronvolt (keV) range for Auger electrons, $\beta^{-}, \beta^{+}$particles, the probability of interaction with high- $Z$ nanoparticles can be significantly higher than that for an external photon beam, which is typically in the mega-electronvolt (MeV) energy range. The interaction probability is also increased by the close proximity of the radiation source to the nanoparticles. Thus, radio-enhancement by nanoparticles should be more significant for internal radionuclide therapy than for external beam radiotherapy (Gholami et al. 2019).

In addition, theranostic radioisotopes such as ${ }^{67,64} \mathrm{Cu}\left(\beta^{-}, \beta^{+}, \gamma\right),{ }^{90} \mathrm{Y}\left(\beta^{-}, \beta^{+}\right)$and ${ }^{177} \mathrm{Lu}\left(\beta^{-}, \gamma\right)$ decay with multiple radiation species enabling them to deliver simultaneous in vivo dose quantification by utilizing nuclear imaging (e.g. PET).

The inherent MRI contrast afforded by radio-labelled SPIONs in addition to PET imaging and delivery of a therapeutic radiation dose delivers a compelling theranostic platform. 


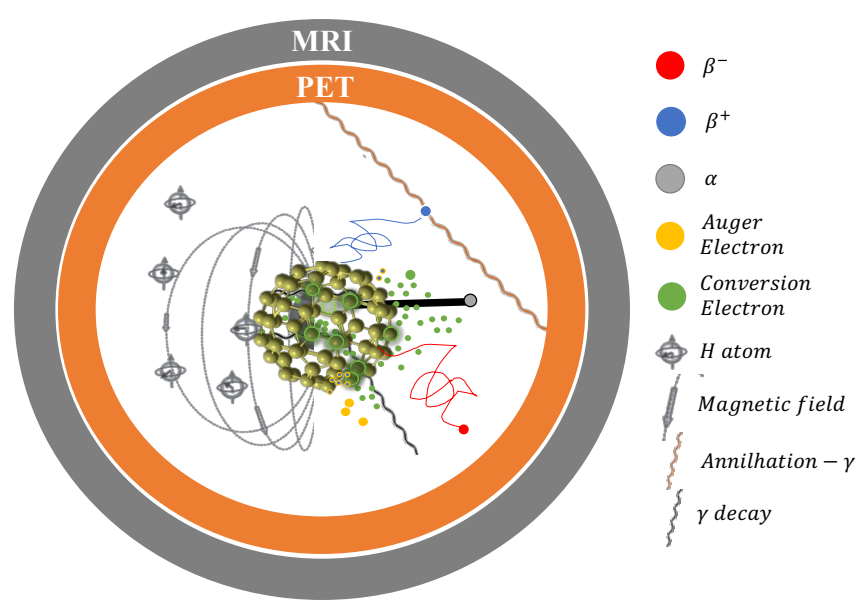

Figure 1. Schematic diagram of a radiolabelled SPION platform for internal radiotherapy and multimodal imaging.

\section{Concluding remarks}

Radiotherapy enhancement can be achieved with nanoparticles incorporating MRI contrast, exploiting some of the latest and most exciting innovations in the radiotherapy space: MRI-linacs; image-guided particle therapy; and radionuclide theranostics. SPION-enhanced MRI-guided particle therapy, in particular, presents an exciting concept that integrates several advanced technologies that up until now have been studied disparately. Feasibility studies are needed to demonstrate the potential benefits of this new paradigm.

\section{References}

Boros, E., et al. (2015), 'Chelate-free metal ion binding and heat-induced radiolabeling of iron oxide nanoparticles', Chem Sci, 6 (1), 225-36.

Choi, G. H., et al. (2012), 'Photon activated therapy (PAT) using monochromatic synchrotron X-rays and iron oxide nanoparticles in a mouse tumor model: feasibility study of PAT for the treatment of superficial malignancy', Radiat Oncol, 7, 184.

Ersahin, D., Doddamane, I., and Cheng, D. (2011), 'Targeted radionuclide therapy', Cancers (Basel), 3 (4), 3838-55.

Fornell, D. (2018), 'The Debate Over Gadolinium MRI Contrast Toxicity'.

Gholami, Y., Maschmeyer, R., and Kuncic, Z. (2019), 'Radio-enhancement effects by radiolabeled nanoparticles', Sci Rep, (Submitted). 
Gholami, Y., et al. (2015), 'Stochastic simulation of radium-223 dichloride therapy at the sub-cellular level', Phys Med Biol, 60 (15), 6087-96.

Iv, M., et al. (2015), 'Clinical applications of iron oxide nanoparticles for magnetic resonance imaging of brain tumors', Nanomedicine (Lond), 10 (6), 993-1018.

Kim, J. K., et al. (2012), 'Enhanced proton treatment in mouse tumors through proton irradiated nanoradiator effects on metallic nanoparticles', Phys Med Biol, 57 (24), 8309-23.

Kuncic, Z. and Lacombe, S. (2018), 'Nanoparticle radio-enhancement: principles, progress and application to cancer treatment', Phys Med Biol, 63 (2), 02TR01.

Liney, G. P., et al. (2018), 'MRI-Linear Accelerator Radiotherapy Systems', Clin Oncol (R Coll Radiol), 30 (11), 686-91.

Liney, G. P., et al. (2019), 'Technical Note: The first live treatment on a 1.0 Tesla inline MRI-linac', Med Phys, 46 (7), 3254-58.

Liu, Y., et al. (2018), 'Metal-based NanoEnhancers for Future Radiotherapy: Radiosensitizing and Synergistic Effects on Tumor Cells', Theranostics, 8 (7), 1824-49.

McMahon, S. J., Paganetti, H., and Prise, K. M. (2016), 'Optimising element choice for nanoparticle radiosensitisers', Nanoscale, 8 (1), 581-9.

Oborn, B. M., et al. (2017), 'Future of medical physics: Real-time MRI-guided proton therapy', Med Phys, 44 (8), e77-e90.

Rudek, B., et al. (2019), 'Radio-enhancement by gold nanoparticles and their impact on water radiolysis for x-ray, proton and carbon-ion beams', Phys Med Biol.

Schuemann, J., et al. (2016), 'Roadmap to Clinical Use of Gold Nanoparticles for Radiation Sensitization', Int J Radiat Oncol Biol Phys, 94 (1), 189-205.

Verry, C., et al. (2019), 'Treatment of multiple brain metastases using gadolinium nanoparticles and radiotherapy: NANO-RAD, a phase I study protocol', BMJ Open, 9 (2), e023591.

Weinstein, J. S., et al. (2010), 'Superparamagnetic iron oxide nanoparticles: diagnostic magnetic resonance imaging and potential therapeutic applications in neurooncology and central nervous system inflammatory pathologies, a review', J Cereb Blood Flow Metab, 30 (1), 15-35.

Yuan, H., et al. (2018), 'Heat-induced radiolabeling and fluorescence labeling of Feraheme nanoparticles for PET/SPECT imaging and flow cytometry', Nat Protoc, 13 (2), 392-412.

Zhang, L., et al. (2010), 'Delivery of therapeutic radioisotopes using nanoparticle platforms: potential benefit in systemic radiation therapy', Nanotechnol Sci Appl, 3, 159-70. 


\title{
Gadolinium Nanoparticles for Imaging and
}

\section{Therapy in Oncology}

\author{
François Lux, ${ }^{1,2,3}$ Ross Berbeco, ${ }^{4}$ Olivier Tillement ${ }^{1,2}$ \\ ${ }^{1} \mathrm{NH}$ TherAguix SAS, Villeurbanne, France \\ 2Univ Lyon Université Claude Bernard Lyon 1, CNRS, Institut Lumière Matière, LYON, France \\ ${ }^{3}$ Institut Universitaire de France (IUF), Paris, France \\ ‘Brigham and Women's Hospital, Dana-Farber Cancer institute and Harvard Medical School, Boston, MA USA
}

\section{Gadolinium as molecular agent.}

Gadolinium is the most frequently used lanthanide in the clinic essentially as positive contrast agent for magnetic resonance imaging (MRI) since the 80s. A molecular agent (Motexafin gadolinium) has been used in combination with radiotherapy for treatment of glioma, non-small cell lung cancer and brain metastases. ${ }^{1}$ Despite good tolerance of the treatment and encouraging preclinical results, motexafin gadolinium did not succeed in increasing significantly overall survival.

\section{Metal based nanoparticles for radiotherapy.}

Most of the studies performed on radiosensitization used nanoparticles taking advantage of gathering high $Z$ elements in a single object. The potential of this strategy for enhancing the efficacy of radiotherapy has been shown even at very small concentrations of NPs in tumors (ppm range) associated with enhancement factors varying from 10 to $100 \%$ using different elements. ${ }^{2,3}$ McMahon et al. have proposed a nanoscale dose deposition mechanism that leads to important formation of radical species in direct vicinity of the nanoparticles. ${ }^{4}$ Despite intense research, only two nanoparticles have been accepted for clinical trials as radiosensitizers for the moment: (i) NBTXR3 (hafnium oxide NPs) administered locally ${ }^{5}$ and AGuIX (polysiloxane based NPs surrounded by gadolinium chelates) administered intravenously. ${ }^{6}$

\section{Why develop ultrasmall nanoparticles for radiosensitization ?}

Development of ultrasmall NPs with a hydrodynamic diameters of less than $5 \mathrm{~nm}$ for biomedical applications is relatively recent due to different reasons: (i) NPs have been initially developed for drug delivery needing large cargo for chemotherapeutic agents, (ii) the enhanced permeability and retention effect was described to be limited to larger NPs (HD > $10 \mathrm{~nm}$; mass $>40 \mathrm{kDa})^{7}$ and is limited to uptake around $1 \%$ of the administered dose, ${ }^{8}$ (iii) larger NPs are characterized by higher interaction with X-Rays and (iv) display longer circulation time in the bloodstream, (v) difficulties to gather imaging and therapeutic functionalities in smallest nano-objects. 
However, advantages of ultrasmall hybrid nanoparticles over regular nanoparticles may be key points for their further rapid translation into the clinic: (i) large nanoparticles are often severely accumulated in organs like liver and spleen ${ }^{9}$ while ultrasmall NPs are rapidly eliminated by the kidneys after intravenous administration,,$^{10}$ (ii) prolonged accumulation in organs for large nanoparticles can be associated with toxicity due to presence of metals, (iii) smaller nanoparticles are associated with better penetration in tumors, ${ }^{11}$ (iv) the size of NPs has also tremendous impact on interaction with XRays, indeed for larger nanoparticles most of ionizing events like secondary electrons occur in the bulk of the NPs drastically reducing deposited dose around the NPs. ${ }^{12}$

\section{Why use gadolinium-based nanoparticles for image guided radiotherapy?}

Our team has shown gadolinium to be of interest for radiosensitization by developing AGuIX NPs. These NPs act as an efficient radiosensitizer under different types of irradiations (photons from keV to $\mathrm{MeV}$, protons or carbons), are efficiently uptaken in tumors by the EPR effect after intravenous administration in animals or in patients with retention for more than a week (particularly adapted for fractionated radiotherapy) and are eliminated effectively by the renal clearance. ${ }^{8}$ Gadolinium is a choice element for its MRI contrast agent properties to better/delineate the tumor before radiotherapy which is a key factor for modern radiotherapy. Indeed, the new generation of radiotherapeutic techniques has led to the possibility to deliver very precisely increased dose in the tumor. ${ }^{13}$ In these conditions, the delivery of the dose is challenging due to uncertainties of imaging, requiring precise delineation of tumors to avoid unwanted irradiation of surrounding tissues especially for tumors located close to organs at risk. The use of MRI is increasing considerably for radiotherapy planning due to its excellent contrast in soft tissues in comparison with CT (computed tomography) and the absence of ionizing radiation especially for pediatric cancer. ${ }^{14}$

In this perspective, the development of MRI/LINAC is a real opportunity that synergizes very well with gadolinium based compounds. Two apparatus are now on the market and display magnetic fields of $0.35 \mathrm{~T}$ (ViewRay) and $1.5 \mathrm{~T}$ (Elekta), ${ }^{15}$ which are relatively low in comparison to new MRI apparatus resulting in lower signal-to-noise ratios and resolution. These apparatus will highly benefit from positive contrast agents, especially nanoparticles like AGuIX that present two to three times higher longitudinal relaxivities in comparison to commercial molecular agents. In our opinion, the association of MRI-LINAC with gadolinium/based nanoparticles creates synergies and can become the new standard of care for different cancer indications needing better targeting.

\section{Conflict of interest}


FL and OT have to disclose the patent WO2011/135101. OT has to disclose the patent WO2009/053644. These patents protect the AGuIX ${ }^{\circledR}$ NPs described in this publication. FL and OT are employees from NH TherAguix and possess shares of this company.

\section{References:}

${ }^{1}$ D. G. Brachman, S. L. Pugh, L. S. Ashby, T. A. Thomas, E. M. Dunbar, S. Narayan, H. I. Robins, J. A. Bovi, J. K. Rockhill, M. Won, W. P. Curran, Phase 1/2 trials of Temozolomide, Motexafin Gadolinium, and 60Gy fractionated radiation for newly diagnosed supratentorial glioblastoma multiforme: final results of RTOG 0513, Int. J. Radiat. Oncol. Biol. Phys., 2015, 91, 961-967.

${ }^{2}$ F. Lux, V.-L. Tran, E. Thomas, S. Dufort, F. Rossetti, M. Martini, C. Truillet, T. Doussineau, G. Bort, F. Denat, F. Boschetti, G. Angelovski, A. Detappe, Y. Crémillieux, N. Mignet, B.-T. Doan, B. Larrat, S. Meriaux, E. Barbier, S. Roux, P. Fries, A. Muller, M. -C. Abadjian, C. Anderson, E. Canet-Soulas, P. Bouziotis, M. Barberi-Heyob, C. Frochot, C. Verry, J. Balosso, M. Evans, J. Sidi-Boumedine, M. Janier, K. Butterworth, S. McMahon, K. Prise, M.-T. Aloy, D. Ardail, C. Rodriguez-Lafrasse, E. Porcel, S. Lacombe, R. Berbeco, A. Allouch, J.-L. Perfettini, C. Chargari, E. Deutsch, G. Le Duc, O. Tillement, "AGuIX ${ }^{\circledast}$ from bench to bedside - Transfer of an ultrasmall theranostic gadolinium-based nanoparticle to clinical medicine ", Br. J. Radiology, 2018, 91, 20180365.

${ }^{3}$ K. Ricketts, R. Ahmad, L. Beaton, B. Cousins, K. Critchley, M. Davies, S. Evans, I. Fenuyi, A. Gavriilidis, Q. J Harmer, D. Jayne, M. Jefford, M. Loizidou, A. Macrobert, S. Moorcroft, I. Naasani, Z. Yuin Ong, K. M. Prise, S. Rannard, T. Richards, G. Schettino, R. A Sharma, O. Tillement, G. Wakefield, N. R. Williams, E. Yaghini, G. Royle, Recommendations for clinical translation of nanoparticle-enhanced radiotherapy, Br. J. Radiol., 2018, 91, 20180325.

${ }^{4}$ S. J. Mc Mahon, H. Pragnetti, K. M. Prise, Optimising element choice for nanoparticle radiosensitizers, Nanoscale, 2016, 8, 581-589.

${ }^{5}$ S. Bonvalot, C. Le Pechoux, T. De Baere, G. Kantor, X. Buy, E. Stoekle, P. Terrier, P. Sargos, J. M. Coindre, N. Lassau, R. Ait Sarkouh, M. Dimitriu, E. Borghi, L. Levy, E Deutsch, J. C. Soria, First-in-human study testing a new radioenhancer using nanoparticles (NBTXR3) activated by radiation therapy in patients with locally advanced soft tissue sarcomas, Clin. Cancer Res., 2017, 23, 908-917. 
${ }^{6}$ C. Verry, L. Sancey, S. Dufort, G. Le Duc, C. Mendoza, F. Lux, S. Grand, J. Arnaud, J. L. Quesada, J. Villa, O. Tillement, J. Balosso, Treatment of multiple brain metastases using gadolinium nanoparticles and radiotherapy : NANO-RAD, a phase I study protocol, BMJ Open, 2019, 11, e023591.

${ }^{7}$ R. Li, K. Zheng, C. Yuan, Z. Chen, M. Huang, Be active or not : the relative contribution of active and passive tumor targeting of nanomaterials, Nanotheranostics, 2017, 1, 346-357.

${ }^{8}$ L. A. Bennie, H. O. McCarthy, J. A. Coulter, Enhanced nanoparticle delivery exploiting tumourresponsive formulation, Cancer Nanotechnol., 2018, 9, 10.

${ }^{9}$ M. Yu, C. Zhou, L. Liu, S. Zhang, S. Sun, J. D. Hankins, X. Sun, J. Zheng, Interactions of renal-clearable nanoparticle gold naoparticles with tumor-microenvironments: vasculature and acidity effect, Angew. Chem. Int. Ed., 2017, 56, 4314-4319.

${ }^{10}$ L. Sancey, S. Kotb, C. Truillet, F. Appaix, A. Marais, E. Thomas, B. van der Sanden, J. P. Klein, B. Laurent, M. Cottier, R. Antoine, P. Dugourd, G. Panczer, F. Lux, P. Perriat, V. Motto-Ros, O. Tillement, Long-term in vivo clearance of gadolinium-based AGuIX nanoparticles and their biocompatibility after systemic injection, ACS Nano, 2015, 24, 2477-2488.

${ }^{11}$ L. Wang, J. Huang, H. Chen, H. Wu, Y. Xu, Y. Li, H. Yi, Y. A. Wang, L. Yang, H. Mao, Exerting enhanced permeability and retention effect driven delivery by ultrafine oxide nanoparticles with T1-T2 switchable magnetic resonance imaging contrast, ACS Nano, 2017, 11, 4582-4592.

12 K. Haume, S. Rosa, S. Grellet, M. A. Smialek, K. T. Butterworth, A. V. Solov'yov, K. M. Prise, J. Golding, N. J. Mason, Gold nanoparticles for cancer radiotherapy : a review, Cancer Nanotechnol., 2016, 7, 8 .

${ }^{13}$ L. Beaton, S. Bandula, M. N. Gaze, R. A. Sharma, How rapid advances in imaging are defining the future of precision radiation oncology, Br. J. Cancer, 2019, doi: 10.1038/s41416-019-0412-y.

${ }^{14}$ M. A. Schmidt, G. S. Payne, Radiotherapy planning using MRI, Phys. Med. Biol., 2015, 60, R323R361.

${ }^{15}$ G. P. Liney, B. Whelan, B. Oborn, M. Barton, P. Keall, MRI-Linear accelerator radiotherapy systems, Clinical Oncology, 2018, 30, 686-691. 


\title{
lodine Nanoparticles
}

\author{
James F. Hainfeld ${ }^{1 *}$, Sharif M. Ridwan ${ }^{2}$, Yaroslav Stanishevskiy ${ }^{1}$, Henry M. Smilowitz ${ }^{2}$ \\ ${ }^{1}$ Nanoprobes, Yaphank, NY, USA \\ 2 University of Connecticut Health Center, Farmington, CT
}

Status

Radiotherapy (RT) is used in about $70 \%$ of all cancer treatments. High atomic number (Z) radiosensitization is an intriguing strategy for improving RT effectiveness and could therefore benefit many patients. Tremendous efforts have explored and advanced implementation, including theoretical analyses, chemical syntheses of novel high-Z nanoparticles, in vitro and in vivo experimental studies, even some with patients. However, progression to routine clinical success is still yet to be achieved.

\section{Current and Future Challenges}

For optimal use the requirements should include: 1) use of kV X-rays (Cho, 2005, Pignol and Lechtman, 2012,), 2) low toxicity, 3) achieving $>0.5 \%$ of the heavy atom concentration (by weight) in the tumor (Loughery et al., 2019), and 4) use of IV rather than intratumoral injection (Smilowitz et al., 2018). However, not meeting some of these requirements may still result in some patient benefit. Highly loading tumors by IV delivery (requirements $2 \& 3$ ) is challenging since only a fraction of the injected dose will home to the tumor, even with targeting. This means that the material must be non-toxic at very high levels (grams/kg administration), a failure of most nanoparticles. Also, maximal IV tumor loading takes many circulatory passes and typically takes at least a day. The nanoparticle must therefore be larger than the kidney filter ( $\sim 5 \mathrm{~nm})$ and have a long blood half-life, also avoiding rapid liver/spleen depletion. Very few nanoparticles have met these requirements. For example, gold nanoparticles satisfy many of the above mentioned requirements, but have very poor whole body clearance $(<10 \%$ liver clearance in 6 months, Sadauskas et al., 2009) and permanently discolor the skin at the high levels needed (Hainfeld et al., 2018).

\section{Advances in Science and Technology to meet the Challenges}

lodine is a candidate heavy element and the successful development of non-toxic X-ray contrast media has led to radiosensitization clinical trials, originally by Norman and collaborators (Rose et al., 1999) using a modified CT, and later by Adam and collaborators (Adam et al., 2016) using a synchrotron (tumor concentration reaching $0.3 \%$ ). A deficiency with this approach is the rapid kidney clearance 
which compromises tumor loading and tumor to non-tumor ratios. Our solution was the synthesis of iodine nanoparticles (INPs, Hainfeld et al. 2018) which are non-toxic at $7 \mathrm{~g}$ iodine/kg, $20 \mathrm{~nm}$ in size, and show a blood half-life of 40 hours (2.7 days). The INPs (Fig. 1) are colorless and do not discolor skin and show slow but steady whole body clearance (>90\% over 15 months). These INPs were found to specifically load orthotopic glioma brain tumors in mice to $0.6 \%$ and showed a doubling or more median life extension after kV irradiation compared to irradiation without the INPs. Improvements may be expected with active targeting and treatment of brain metastases where we have observed much higher levels of INP loading. We also found that INP-enhanced RT of advanced othotopic human gliomas in mice synergizes with DOXIL chemotherapy (Hainfeld et al., 2019, Fig. 1e) raising the possibility that INPRT may benefit other combination therapies including chemo-, immune- and oncolytic virus therapies.

\section{Concluding Remarks}

lodine nanoparticles therefore appear to overcome problems with many other high atomic number nanoparticles and should help advance high- $Z$ nanoparticle radiosensitization to clinical use and patient benefit.
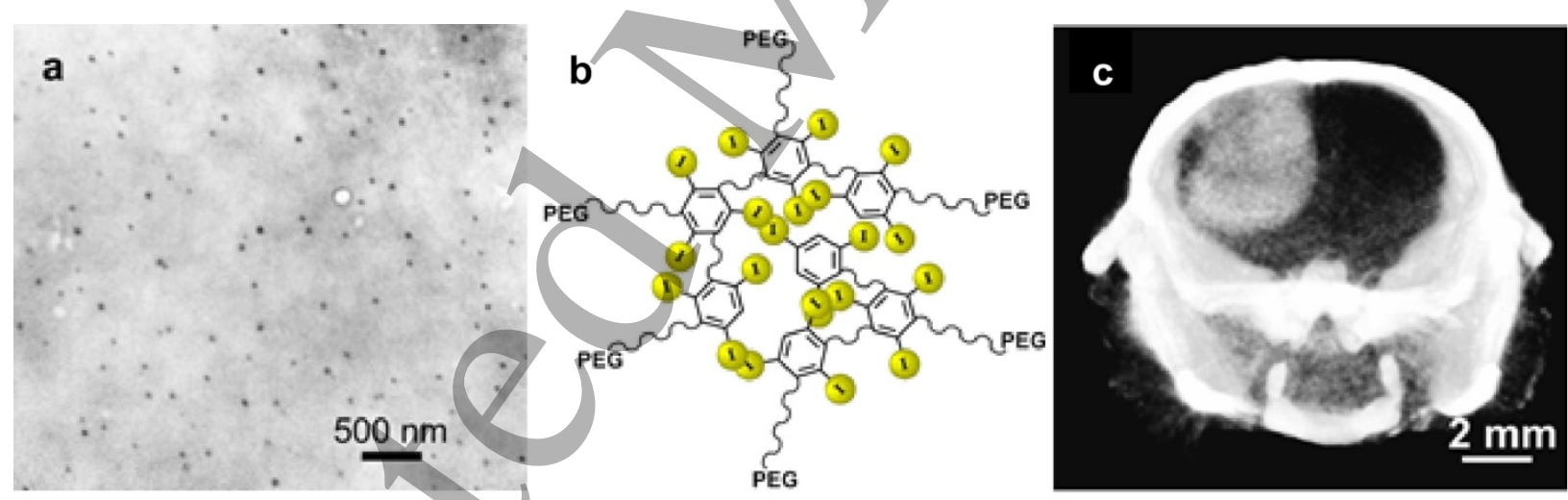

d Survival time increased 2.4 times over standard treatment

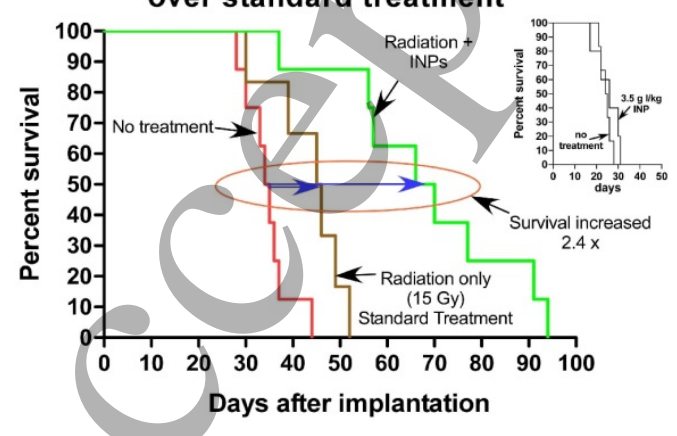

e

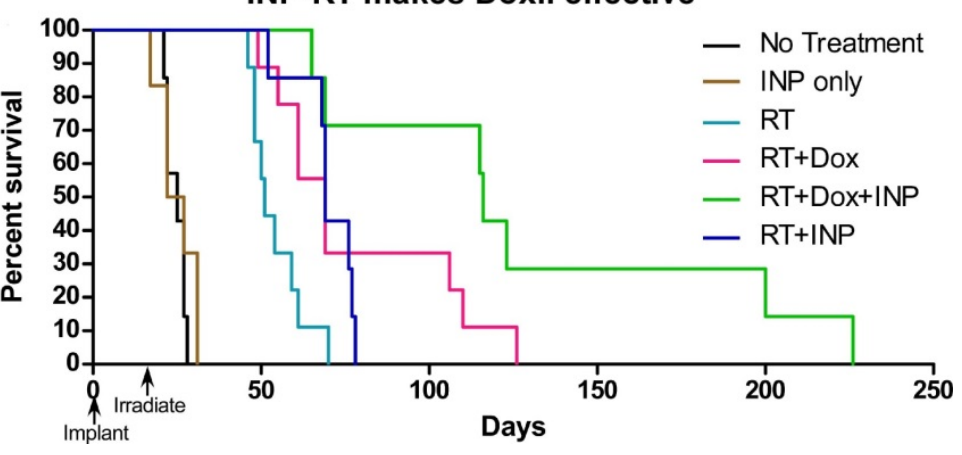


Figure 1. lodine nanoparticles: (a): Electron micrograph; (b):schematic; (c): accumulation in U87 glioma in mouse (microCT), (d): therapeutic efficacy, and e) INP-RT greatly increases Doxil effectiveness. Taken from Hainfeld et al., 2018, 2019.

\section{References}

Adam, J.F., J. Balosso, M. Renier, H. Elleaume, F. Estève, P. Berkvens, C. Nemoz, T. Brochard, A. Tessier, C. Verry, J.F. Le Bas, Synchrotron Stereotactic Radiation Therapy: A Report on Phase 1/2 Clinical Trial Achievements, Ongoing Developments, and Long-Term Prospects, In International Journal of Radiation Oncology*Biology*Physics, Volume 96, Issue 2, Supplement, 2016, Pages E624-E625, ISSN 0360-3016, https://doi.org/10.1016/j.jjrobp.2016.06.2193.

Cho, S.H. Estimation of tumour dose enhancement due to gold nanoparticles during typical radiation treatments: a preliminary Monte Carlo study. Phys Med Biol. 2005;50(15):N163-173.

Hainfeld JF, Ridwan SM, Stanishevskiy Y, Panchal R, Slatkin DN, Smilowitz HM. lodine nanoparticles enhance radiotherapy of intracerebral human glioma in mice and increase efficacy of chemotherapy. Sci Rep. 2019 Mar 14;9(1):4505. doi: 10.1038/s41598-019-41174-5. PMID: 30872755.

Hainfeld JF, Ridwan SM, Stanishevskiy Y, Smilowitz NR, Davis J, Smilowitz HM. Small, Long Blood HalfLife lodine Nanoparticle for Vascular and Tumor Imaging. Sci Rep. 2018 Sep 14;8(1):13803. doi: 10.1038/s41598-018-31940-2. PMID: 30218059.

Hainfeld JF, Ridwan SM, Stanishevskiy Y, Smilowitz NR, Davis J, Smilowitz HM. Small, Long Blood HalfLife lodine Nanoparticle for Vascular and Tumor Imaging. Sci Rep. 2018 Sep 14;8(1):13803. doi: 10.1038/s41598-018-31940-2. PMID: 30218059.

Loughery Brian, Robert Halford, Kathryn Dess, Joseph Rakowski, Mary Cox, Joseph Koh, Alan Mayville, Jeffery Riess and Michael Snyder. A proof of concept kilovoltage intensity modulated radiotherapy platform for the treatment of glioblastoma multiforme. 2019 Biomed. Phys. Eng. Express 5055013

Pignol, J-P, and Lechtman, Eli. Reply to Comment on 'Implications on clinical scenario of gold nanoparticle radiosensitization in regards to photon energy, nanoparticle size, concentration and location', 2012 Phys. Med. Biol. 57291.

Rose J H, Norman A, Ingram M, Aoki C, Solberg T, and Mesa 1999 A First radiotherapy of human metastatic brain tumors delivered by a computerized tomography scanner (CTRx) Int. J. Radiat. Oncol. Biol. Phys. 45 1127-32.

Sadauskas E, Danscher G, Stoltenberg M, Vogel U, Larsen A, et al. (2009). Protracted elimination of gold nanoparticles from mouse liver. Nanomedicine 5:162-169. 
1

2

3

4

5

6

7

8

9

10

11

12

13

14

15

16

17

18

19

20

21

22

23

24

25

26

27

28

29

30

31

32

33

34

35

36

37

38

39

40

41

42

43

44

45

46

47

48

49

50

51

52

53

54

55

56

57

Smilowitz, HM, Alexandra Meyers, Khalil Rahman, Nathaniel A Dyment, Dan sasso, Crystal Xue, Douglas Oliver, Alexander Lichtler, Xiaomeng Deng, Sharif M Ridwan, Lauren J Tarmu, Qian Wu, Andrew Salner, Keytan R Bulsara, Daniel N Slatkin, James F Hainfeld. Intravenously-injected gold nanoparticles (AuNPs) access intracerebral F98 rat gliomas better than auNPs infused directly into the tumor site by convection enhanced delivery. International Journal of Nanomedicine, 2018. 


\title{
Optical and Photoacoustic Imaging of GNPs
}

\author{
Dmitry Nevozhay ${ }^{1,2}$ and Konstantin V. Sokolov ${ }^{1,3,4}$ \\ ${ }^{1}$ Department of Imaging Physics, The University of Texas MD Anderson Cancer Center, \\ Houston, TX 77030, USA \\ ${ }^{2}$ School of Biomedicine, Far Eastern Federal University, Vladivostok, 690950, Russia \\ ${ }^{3}$ Department of Bioengineering, Rice University, Houston, TX 77005, USA \\ ${ }^{4}$ Department of Biomedical Engineering, The University of Texas at Austin, Austin, TX \\ 78712, USA \\ E-mail:ksokolov@mdanderson.org
}

Keywords: gold, nanoparticles, imaging, optical, photoacoustics

\section{Status}

The use of GNPs in biomedical optical and photoacoustic imaging was facilitated by few key properties. First, gold has been known to be non-toxic and biocompatible since antiquity. Second, current methods allow production of GNPs in various sizes and shapes which enables tuning of their physico-chemical properties. Third, GNPs exhibit strong surface plasmon resonances in visible and near-infrared (NIR) regions that are ideally suited for imaging of biological specimen. Lastly, the surface of GNPs can be easily modified to introduce various functionalizations including targeting moieties or different coatings that can alter biological interactions.

We reported one of the first studies demonstrating optical imaging using GNPs conjugated with anti-EGFR antibodies for specific labeling of cancer cells in cultures and biopsies from cancer patients (Sokolov et al., 2003). This approach is based on the ability of GNPs to strongly scatter light due to excitation of surface plasmon resonances, which are dependent on size and shape of GNPs, and also physical proximity between the individual particles. We showed that molecular specific interactions of immunotargeted GNPs with cells can lead to formation of closely spaced GNP assemblies that are associated with a strong red shift in GNPs scattering spectra due to the plasmon resonance coupling (Sokolov et al., 2003, Aaron et al., 2009). This phenomenon was used for optical imaging of cancer cells with high contrast in cell cultures, tissue phantoms and animal models (Aaron et al., 2007, Aaron et al., 2009). A number of elegant studies used the effect of plasmon resonance coupling to image interactions and trafficking of biological molecules with sub-diffractional spatial resolution including dynamic behavior of integrin molecules on a cellular cytoplasmic membrane (Rong et al., 2008), transport of cell membrane receptors to endosomes (Aaron et al., 2009), and monitoring of caspase- 
3 activity in live cells (Jun et al., 2009). In parallel with the imaging studies, a theoretical framework was introduced to estimate distances between coupled gold nanoparticles that provided the foundation for the concepts of "plasmonic ruler" and "plasmon coupling microscopy" (Wu and Reinhard, 2014) as new tools in biological imaging.

GNPs also exhibit a strong two-photon luminescence (TPL) providing another dimension in biological applications that was first demonstrated in imaging of a single gold nanorod (GNR) in a mouse ear blood flow (Wang et al., 2005). Subsequently, TPL with GNPs was applied for molecular imaging of cancer cells (Durr et al., 2007). GNPs with NIR extinction spectra are ideally suited for TPL imaging in vivo due to increased tissue penetration in the NIR. Further, GNPs exhibit a very short, in order of tens of picoseconds, fluorescence lifetime under two-photon excitation as compared to nanoseconds for organic fluorophores that can enable high contrast in fluorescence lifetime imaging microscopy (FLIM) of cells and tissue (Zhang et al., 2010).

The absorbance property of GNPs was used to enable sensitive molecular imaging in optical coherence tomography (OCT) (Lapierre-Landry et al., 2017). In this method a modulated heating laser is used to induce highly localized oscillating changes in the refractive index of media in immediate vicinity of a GNP. These oscillations are detected with high sensitivity by OCT and allow detection of GNPs with high contrast (Lapierre-Landry et al., 2017).

Introduction of photoacoustics (PA), a hybrid modality with optical excitation and ultrasound detection, provided an imaging depth of several centimeters in tissue. PA signal is generated by a thermoelastic expansion of media around light-absorbing molecules or nanoparticles. GNPs found their application in PA imaging (PAI) due to their exceptionally high absorbance that is several orders of magnitude higher than that of organic dyes. Initial studies demonstrated the use of gold nanoshells coated with polyethylene glycol (PEG) for visualization of the vasculature in a rat brain (Wang et al., 2004). Subsequently, we showed that plasmon resonance coupling can also be used in PA for highly sensitive detection of labeled cancer cells using antibody targeted GNPs (Mallidi et al., 2007). Later, we used plasmon resonance coupling to achieve an unprecedented sensitivity of just few tens of cancer cells in lymph node micrometastases in animal models of head and neck cancer (Luke et al., 2014). PAl with GNPs also showed significant promise in cell tracking applications including mesenchymal stem cells (Donnelly et al., 2018). Further, GNPs were used in multimodal strategies for tumor detection using PAI and margin delineation by Surface-Enhanced Raman Scattering (SERS) (Kircher et al., 2012). 


\section{Current and future challenges}

Most of NIR absorbing GNPs showed unfavorable pharmakokinetics (PK) with a low accumulation at a target site, e.g. tumor, following i.v. administration. This behavior could be associated with a relatively big size, typically $>60 \mathrm{~nm}$, and instability of GNP coating in blood.

Generally, particles with size $<5 \mathrm{~nm}$ are quickly excreted via kidneys, usually within several hours, whereas an increase in size is associated with a higher propensity of uptake by the reticuloendothelial system (RES) and an increased accumulation in liver and spleen where GNPs can reside for months while slowly being excreted via the hepatobiliary route (Poon et al., 2019).

Coating of GNPs with PEG or other biocompatible polymers is the most common approach to reduce opsonization and subsequent uptake of GNPs by RES. The efficiency of this protection is dependent on the density of PEG molecules on the GNP surface . However, PEGylation does not eliminate nonspecific uptake by RES as polymer molecules can be displaced by physiological concentrations of cysteine and cystine (Larson et al., 2012).

High levels of accumulations and a long residence time of NIR-absorbing GNPs in liver and spleen in combination with non-biodegradable nature of gold brings up concerns about potential long termtoxicity. Most studies report no significant adverse effects of GNPs ranging in size from 12 to $85 \mathrm{~nm}$. Comprehensive evaluations that take into account the whole nanoparticle construct including the coating and the geometry (e.g., size and shape) of the gold core most likely will be required for each GNP that is considered for a clinical translation.

Another common problem of NIR-absorbing GNPs is their decreased photostability due to thermal degradation caused by laser irradiation with a high peak intensity that leads to a decrease of the photoacoustic signal (Chen et al., 2010).

\section{Advances in science and technology to meet challenges}

The thermal instability of GNPs can be addressed by a combination of advanced GNP synthesis and surface modification techniques. Specific examples include silica coating of GNRs (Chen et al., 2010) as well as synthesis of miniature GNRs (Chen et al., 2019).

To address challenges of clinical translation of GNPs, we introduced the concept of biodegradable plasmonic nanoparticles that consist of $5 \mathrm{~nm}$ GNPs assembled into sub-100 nm nanoclusters using biodegradable polymers (Tam et al., 2010). In a biological media the nanoclusters can fully dissociate over time into primary $5 \mathrm{~nm}$ particles which are favorable for a rapid clearance form the body.

Delivery of GNPs to tumor can be improved by developing better coatings. We showed that inclusion of a small alkyl chain between the gold surface and the outer PEG layer can greatly improve 
stability of GNPs and diminish their uptake by macrophages up to 5 days (Larson et al., 2012). Another approach is based on the use of erythrocyte membranes as a GNP coating that was shown to decrease GNP uptake by macrophages (Gao et al., 2013).

\section{Concluding remarks}

Intricate dependence of plasmon resonances of GNPs on their geometry, interparticle distance and environment has led to innovative directions in development of biosensors, bioanalytical assays and cell imaging tools. There is also continuous flow of exciting reports of preclinical imaging and therapeutic developments involving GNPs. Future adoption of GNPs in the clinic will rely on optimization of GNPs pharmacokinetics in vivo and a better mechanistic understanding of GNPs toxicity and clearance. Applications of GNPs for intravital imaging, cell tracking, and therapy will likely undergo further exploration with emphasis on multimodal imaging and theranostic approaches.

\section{Acknowledgements}

The authors acknowledge support from Cancer Prevention and Research Institute of Texas (CPRIT RP170314).

\section{References}

AARON, J., NITIN, N., TRAVIS, K., KUMAR, S., COLLIER, T., PARK, S. Y., JOSE-YACAMAN, M., COGHLAN, L., FOLLEN, M., RICHARDS-KORTUM, R. \& SOKOLOV, K. 2007. Plasmon resonance coupling of metal nanoparticles for molecular imaging of carcinogenesis in vivo. J Biomed Opt, 12, 034007. AARON, J., TRAVIS, K., HARRISON, N. \& SOKOLOV, K. 2009. Dynamic imaging of molecular assemblies in live cells based on nanoparticle plasmon resonance coupling. Nano Lett, 9, 3612-8.

CHEN, Y. S., FREY, W., KIM, S., HOMAN, K., KRUIZINGA, P., SOKOLOV, K. \& EMELIANOV, S. 2010. Enhanced thermal stability of silica-coated gold nanorods for photoacoustic imaging and image-guided therapy. Opt Express, 18, 8867-78.

CHEN, Y. S., ZHAO, Y., YOON, S. J., GAMBHIR, S. S. \& EMELIANOV, S. 2019. Miniature gold nanorods for photoacoustic molecular imaging in the second near-infrared optical window. Nat Nanotechnol.

DONNELLY, E. M., KUBELICK, K. P., DUMANI, D. S. \& EMELIANOV, S. Y. 2018. Photoacoustic ImageGuided Delivery of Plasmonic-Nanoparticle-Labeled Mesenchymal Stem Cells to the Spinal Cord. Nano Letters, 18, 6625-6632. 
DURR, N. J., LARSON, T., SMITH, D. K., KORGEL, B. A., SOKOLOV, K. \& BEN-YAKAR, A. 2007. Two-photon luminescence imaging of cancer cells using molecularly targeted gold nanorods. Nano Lett, 7 , 941-5.

GAO, W., HU, C. M., FANG, R. H., LUK, B. T., SU, J. \& ZHANG, L. 2013. Surface functionalization of gold nanoparticles with red blood cell membranes. Adv Mater, 25, 3549-53.

JUN, Y. W., SHEIKHOLESLAMI, S., HOSTETTER, D. R., TAJON, C., CRAIK, C. S. \& ALIVISATOS, A. P. 2009. Continuous imaging of plasmon rulers in live cells reveals early-stage caspase- 3 activation at the single-molecule level. Proc Natl Acad Sci U S A, 106, 17735-40.

KIRCHER, M. F., DE LA ZERDA, A., JOKERST, J. V., ZAVALETA, C. L., KEMPEN, P.J., MITTRA, E., PITTER, K., HUANG, R., CAMPOS, C., HABTE, F., SINCLAIR, R., BRENNAN, C. W., MELLINGHOFF, I. K., HOLLAND, E. C. \& GAMBHIR, S. S. 2012. A brain tumor molecular imaging strategy using a new triple-modality MRI-photoacoustic-Raman nanoparticle. Nature Medicine, 18, 829.

LAPIERRE-LANDRY, M., GORDON, A. Y., PENN, J. S. \& SKALA, M. C. 2017. In vivo photothermal optical coherence tomography of endogenous and exogenous contrast agents in the eye. Sci Rep, 7, 9228.

LARSON, T. A., JOSHI, P. P. \& SOKOLOV, K. 2012. Preventing protein adsorption and macrophage uptake of gold nanoparticles via a hydrophobic shield. ACS Nano, 6, 9182-90.

LUKE, G. P., MYERS, J. N., EMELIANOV, S. Y. \& SOKOLOV, K. V. 2014. Sentinel lymph node biopsy revisited: ultrasound-guided photoacoustic detection of micrometastases using molecularly targeted plasmonic nanosensors. Cancer Res, 74, 5397-408.

MALLIDI, S., LARSON, T., AARON, J., SOKOLOV, K. \& EMELIANOV, S. 2007. Molecular specific optoacoustic imaging with plasmonic nanoparticles. Opt Express, 15, 6583-8.

POON, W., ZHANG, Y. N., OUYANG, B., KINGSTON, B. R., WU, J. L. Y., WILHELM, S. \& CHAN, W. C. W. 2019. Elimination Pathways of Nanoparticles. ACS Nano.

RONG, G., WANG, H., SKEWIS, L. R. \& REINHARD, B. M. 2008. Resolving sub-diffraction limit encounters in nanoparticle tracking using live cell plasmon coupling microscopy. Nano Lett, 8, 3386-93.

SOKOLOV, K., FOLLEN, M., AARON, J., PAVLOVA, I., MALPICA, A., LOTAN, R. \& RICHARDS-KORTUM, R. 2003. Real-time vital optical imaging of precancer using anti-epidermal growth factor receptor antibodies conjugated tó gold nanoparticles. Cancer Res, 63, 1999-2004.

TAM, J. M., TAM, J. O., MURTHY, A., INGRAM, D. R., MA, L. L., TRAVIS, K., JOHNSTON, K. P. \& SOKOLOV, K. V. 2010. Controlled assembly of biodegradable plasmonic nanoclusters for near-infrared imaging and therapeutic applications. ACS Nano, 4, 2178-84. 
WANG, H. F., HUFF, T. B., ZWEIFEL, D. A., HE, W., LOW, P. S., WEI, A. \& CHENG, J. X. 2005. In vitro and in vivo two-photon luminescence imaging of single gold nanorods. Proceedings of the National Academy of Sciences of the United States of America, 102, 15752-15756.

WANG, Y. W., XIE, X. Y., WANG, X. D., KU, G., GILL, K. L., O'NEAL, D. P., STOICA, G. \& WANG, L. V. 2004. Photoacoustic tomography of a nanoshell contrast agent in the in vivo rat brain. Nano Letters, 4, 1689-1692.

WU, L. \& REINHARD, B. M. 2014. Probing subdiffraction limit separations with plasmon coupling microscopy: concepts and applications. Chem Soc Rev, 43, 3884-97.

ZHANG, Y. A., YU, J., BIRCH, D. J. S. \& CHEN, Y. 2010. Gold nanorods for fluorescence lifetime imaging in biology. Journal of Biomedical Optics, 15. 


\section{In Vivo Imaging of Metallic Nanoparticles Using Benchtop X-ray Fluorescence Computed Tomography}

\section{Techniques}

\section{Sang Hyun Cho}

${ }^{1}$ Department of Radiation Physics \& Department of Imaging Physics, The University of Texas MD Anderson Cancer Center, Houston, TX 77030, USA

Author contact: Sang Hyun Cho (scho@mdanderson.org)

\section{Status}

Over the last decade, $\mathrm{x}$-ray fluorescence (XRF) computed tomography (XFCT), traditionally a synchrotron $\mathrm{x}$-ray imaging modality, has also been implemented with ordinary polychromatic $\mathrm{x}$ ray sources (viz., "benchtop XFCT"), aiming to enable routine XFCT imaging of biological objects (e.g., small animals) under typical biomedical laboratory settings. In particular, it was the absence of a proper quantitative imaging tool for small animal studies involving high atomic number $(Z>$ 50) metallic nanoparticles, such as gold nanoparticles (GNPs), which prompted the initial efforts on benchtop XFCT development with small animal-sized phantoms (Cheong et al., 2010; Jones et al., 2012). The feasibility of extending benchtop XFCT beyond GNP imaging has been demonstrated through similar phantom imaging studies using high-Z probes such as platinum, gadolinium and iodine (within the context of multiplexed imaging) (Kuang et al., 2013a; Kuang et al., 2013b), as well as low-Z molybdenum NPs (Hertz et al., 2014).

To date, a clear pathway towards the fulfillment of the aforementioned goal of benchtop XFCT imaging has been well established, for example by the first successful animal imaging study 
(albeit performed postmortem) using an experimental benchtop XFCT system and GNPs (Manohar et al., 2016). More recently, another postmortem animal imaging study has demonstrated the feasibility of benchtop XFCT imaging of mice injected with molybdenum NPs (Larsson et al., 2018). Additionally, very recent publications have reported benchtop XFCT) and XRF imaging/mapping of live mice injected with gadolinium NPs (Zhang et al., 2019) and GNPs (Jung et al., 2020), respectively. Despite these reports and other on-going (unpublished) efforts, in vivo imaging with benchtop XFCT is still in its infancy. In particular, the feasibility of performing whole-body imaging of live animals using benchtop XFCT techniques, while meeting the key requirements for routine applications (to be described below), has yet to be demonstrated.

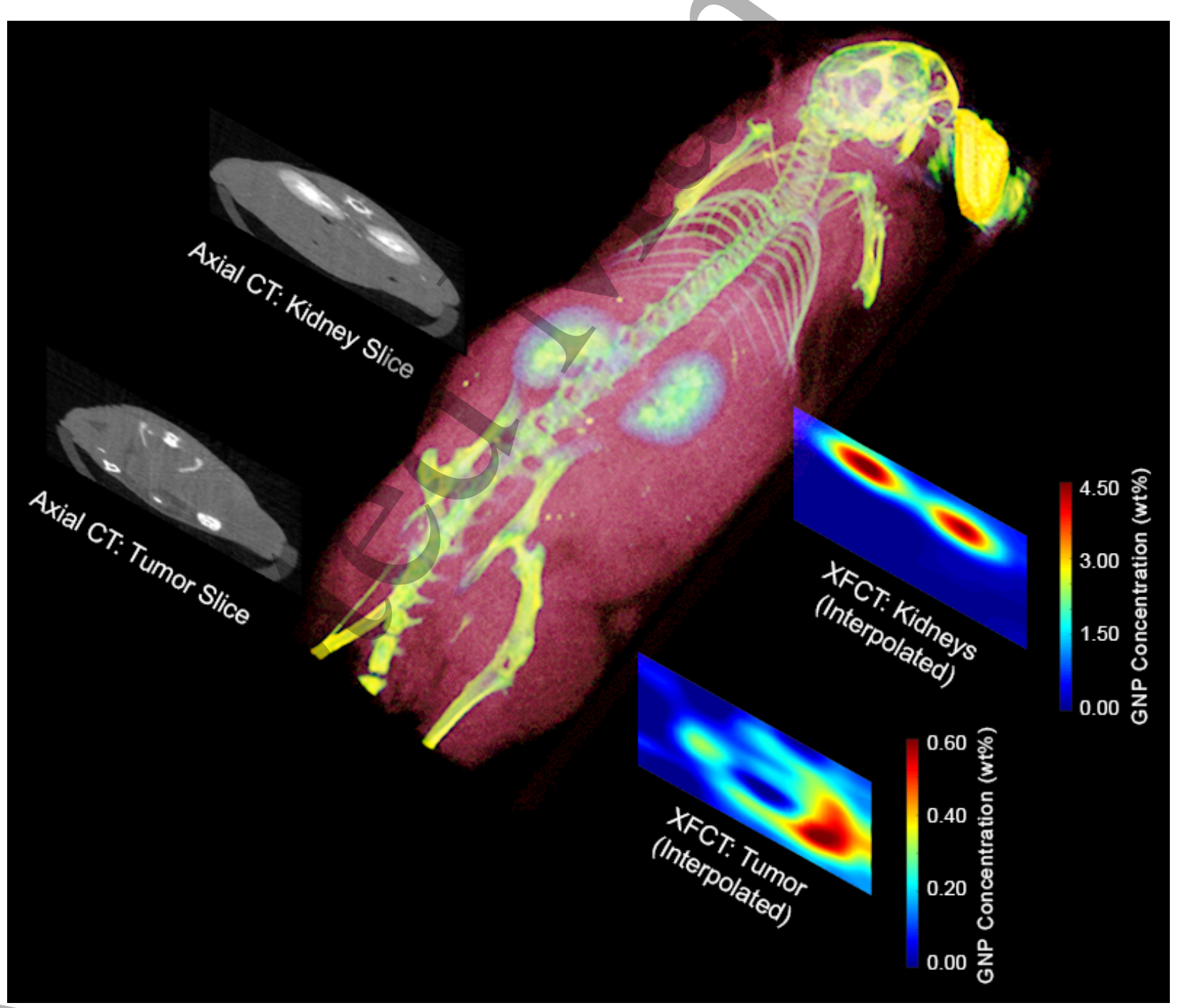


Figure 1. Illustration of the possibility of quantitative multimodal imaging of GNP distributions in an animal using benchtop XFCT and conventional CT together. 3D volume rendering of the mouse from postmortem CT data along with smoothed XFCT images and corresponding axial CT images of the kidney and tumor slices (Reprinted with permission, Manohar et al. 2016).

According to a recent experimental study (Manohar et al., 2018), a practical benchtop XFCT system with transmission CT capability can be built for whole-body imaging of small animals (e.g., 3-cm diameter mice) containing GNPs at relatively low concentrations on the order of several hundred parts-per-million (ppm) (e.g., $0.3 \mathrm{mg}$ of GNPs per gram tissue). In general, such a benchtop XFCT system would be considered acceptable for in vivo imaging of small animals, if it could satisfy the realistic constraints of x-ray dose and scan time/e.g., less than $\sim 40$ cGy and 1 hour per imaging session, respectively) for in vivo imaging.

\section{Current and future challenges}

Compared with synchrotron XFCT, benchtop XFCT is typically performed with relatively higher energy but less intense polychromatic x-ray sources, resulting in less efficient XRF photon production and detection. This aspect directly affects the material detection limit (or system sensitivity) at a given x-ray dose as well as the overall scan time (or data acquisition time) of a benchtop XFCT system. The consequences from this aspect are more pronounced for benchtop XFCT performed with relatively high x-ray tube voltages (e.g., > $100 \mathrm{kVp}$ or average energy of $\sim 80 \mathrm{keV}$ ), due to increased scatter of higher energy photons that complicates the XRF signal extraction from the scatter background. Thus, there is a strong motivation to adopt monochromatic/quasi-monochromatic x-ray sources, if available on a benchtop setting, to overcome the inherent difficulties of benchtop XFCT. For example, published computational studies (Manohar et al., 2014; Ahmad et al., 2014) suggested more than one order of magnitude increase in the XRF signal per a given x-ray dose or the sensitivity, with the use of such x-ray 
Another critical technical challenge arises from the current difficulty in parallel acquisition (using array or pixelated detectors) of XRF signals, which would be essential to accomplish in vivo imaging of small animals using a benchtop XFCT system under the given constraints of x-ray dose and scan time. Additionally, XFCT requires fully spectroscopic data with sufficient energy resolution (e.g., < $1 \mathrm{keV}$ energy bin), in order to achieve highly sensitive quantitative imaging of both known and unknown elements present within the imaging objects. For benchtop XFCT, this requirement has been met reasonably by adopting a commercial, energy-resolving, thermoelectrically-cooled, single-crystal cadmium telluride (CdTe) detector (Cheong et al., 2010). In fact, custom-array detectors (or multiple detectors working in parallel) using this detector can easily be adopted for benchtop XFCT, lowering the scan time and x-ray dose inversely proportional to the number of detectors (Jones et al., 2012). Nonetheless, there is a practical limitation for this approach in terms of the number of detectors that can be deployed into benchtop XFCT setups built on smaller footprints for the purpose of small animal imaging. Thus, despite a few known/expected difficulties (e.g., inferior energy resolution/charge sharing effect), pixelated detectors may be found more advantageous for parallel XRF signal acquisition than arrays of single crystal detectors.

The remaining technological challenges for benchtop XFCT reside mainly with optimization of various hardware components and operating parameters. While not specifically discussed/referenced in this article, numerous investigations have contributed to research advances in these aspects. Additionally, there are a number of issues that are uniquely associated with in vivo imaging but have not been seriously investigated thus far. Such issues include application-specific optimization of metallic NPs (in terms of material/size of NPs as well as 
conjugation of targeting moieties), multimodal (e.g., CT+XFCT) and/or multiplexed imaging, and effects of NP pharmacodynamics/pharmacokinetics on benchtop XFCT scanning and image reconstruction.

\section{Advances in science and technology to meet challenges}

Currently, laser-driven x-ray sources producing sufficiently intense monochromatic x-rays, based on inverse Compton scattering, are available on a relatively small footprint and have been considered for various applications including x-ray CT (Achterhold et al., 2013). Also, other special X-ray sources that offer quasi-monochromatic spectra based on intense XRF lines (von Busch et al., 2005; Hertz et al., 2014) have been used for benchtop XFCT. Although attractive in terms of their x-ray spectra, these sources are associated with some limitations (e.g., x-ray energy and flux/output, size, cost, etc.) similar to those of synchrotron XFCT, which may need to be carefully considered if benchtop XFCT is intended primarily for preclinical imaging. Meanwhile, as practical alternatives, carefully designed metal filters, especially in conjunction with high-power (e.g., > 1 kW) x-ray sources, or crystal-based approaches utilizing the Bragg diffraction (e.g., the use of highly oriented pyrolitic graphite), can also be pursued to tailor the polychromatic x-ray spectrum for benchtop XFCT imaging of high-Z probes including GNPs (Manohar et al., 2014).

While readily available, typical pixelated photon-counting detectors provide only a limited number $(\sim 4-5)$ of rather broad energy bins/windows (in a few keV intervals) and, consequently, may not meet the detector specifications for ideal benchtop XFCT imaging. Nevertheless, there have been attempts to adopt some of them for benchtop XFCT (Yoon et al., 2016; Ren et al., 2016; Li et al., 2017), providing some insight into the technical feasibility and reconfirming some benefits of benchtop XFCT previously demonstrated (e.g. better sensitivity than transmission CT) (Bazalova et al., 2012; Manohar et al., 2016). Overall, these initial attempts have commonly revealed somewhat expected consequences from the insufficient detector energy resolution, most notably 
the difficulty in achieving highly sensitive benchtop XFCT. In theory, such consequences may be avoided or at least mitigated by adopting fully-spectroscopic pixelated detectors providing higher energy resolution than conventional photon-counting detectors (Seller et al., 2011). Nonetheless, it remains to be seen whether or not this expectation can be realized under the typical operating conditions for the whole-body imaging with benchtop XFCT.

Considering the research advances on the two key issues discussed above (as well as other issues mentioned earlier but not specifically discussed here), it will soon be possible to produce a benchtop XFCT system (with simultaneous or sequential CT capability) that can allow for wholebody imaging of live animals while meeting realistic scan time/X-ray dose constraints. A fullyoptimized benchtop XFCT system (with an ordinary polychromatic x-ray source) will likely enable several fold improvement in the sensitivity and scan time (e.g., detection limit of $\sim 100$ ppm of gold and less than $\sim 20$ minutes of scan time, in the case of GNP imaging), compared to the currently available systems.

\section{Concluding remarks}

Benchtop XFCT is now nearing to become a practical preclinical imaging modality. Further improvement beyond the currently anticipated technical specifications would still be possible, depending on future research advances.

\section{Acknowledgments}

This work was supported by the US National Institutes of Health under the award number R01EB020658. The author acknowledges the former and current lab members as well as research collaborators for their invaluable contributions to the development of benchtop XFCT techniques and systems. 


\section{REFERENCES}

Achterhold K, Bech M, Schleede S, Potdevin G, Ruth R, Loewen R and Pfeiffer F 2013 Monochromatic computed tomography with a compact laser-driven X-ray source Scientific Reports 3

Ahmad M, Bazalova M, Xiang L Z and Xing L 2014 Order of Magnitude Sensitivity Increase in X-ray Fluorescence Computed Tomography (XFCT) Imaging With an Optimized Spectro-Spatial Detector Configuration: Theory and Simulation IEEE Trans. Med. Imag. 33 1119-28

Bazalova M, Kuang Y, Pratx G and Xing L 2012 Investigation of X-ray Fluorescence Computed Tomography (XFCT) and K-Edge Imaging IEEE Trans. Med. Imag. 31 1620-7

Cheong S K, Jones B L, Siddiqi A K, Liu F, Manohar N and Cho S H 2010 X-ray fluorescence computed tomography (XFCT) imaging of gold nanoparticle-loaded objects using 110 kVp x-rays Phys. Med. Biol. 55 647-62

Hertz H M, Larsson J C, Lundstrom U, Larsson D H and Vogt C 2014 Laboratory x-ray fluorescence tomography for high-resolution nanoparticle bio-imaging Opt. Lett. 39 2790-3

Jones B L, Manohar N, Reynoso F, Karellas A and Cho S H 2012 Experimental demonstration of benchtop x-ray fluorescence computed tomography (XFCT) of gold nanoparticleloaded objects using lead- and tin-filtered polychromatic cone-beams Phys. Med. Biol. 57 N457-67

Jung S, Kim T, Lee W, Kim H, Kim H S, Im H J and Ye S J 2020 Dynamic In Vivo X-ray Fluorescence Imaging of Gold in Living Mice Exposed to Gold Nanoparticles IEEE Trans. Med. Imag. 39 526-33

Kuang Y, Pratx G, Bazalova M, Meng B W, Qian J G and Xing L 2013a First Demonstration of Multiplexed X-Ray Fluorescence Computed Tomography (XFCT) Imaging IEEE Trans. Med. Imag. 32 262-7

Kuang Y, Pratx G, Bazalova M, Qian J, Meng B and Xing L 2013b Development of XFCT imaging strategy for monitoring the spatial distribution of platinum-based chemodrugs: instrumentation and phantom validation Med. Phys. 40030701

Larsson J C, Vogt C, Vagberg W, Toprak M S, Dzieran J, Arsenian-Henriksson M and Hertz H M 2018 High-spatial-resolution X-ray fluorescence tomography with spectrally matched nanoparticles Phys. Med. Biol. 63

Li L, Zhang S Y, Li R Z and Chen Z Q 2017 Full-field fan-beam x-ray fluorescence computed tomography with a conventional $\mathrm{x}$-ray tube and photon-counting detectors for fast nanoparticle bioimaging Optical Engineering 56

Manohar N, Jones B L and Cho S H 2014 Improving X-ray fluorescence signal for benchtop polychromatic cone-beam x-ray fluorescence computed tomography by incident x-ray spectrum optimization: A Monte Carlo study Medical physics 41101906

Manohar N, Reynoso F J and Cho S H 2018 Technical Note: A benchtop cone-beam x-ray fluorescence computed tomography (XFCT) system with a high-power x-ray source and transmission CT imaging capability Med. Phys. 45 4652-9

Manohar N, Reynoso F J, Diagaradjane P, Krishnan S and Cho S H 2016 Quantitative imaging of gold nanoparticle distribution in a tumor-bearing mouse using benchtop x-ray fluorescence computed tomography Scientific Reports 622079 
Ren L Q, Wu D, Li Y H, Chen W R, Zheng B and Liu H 2016 Optimized acquisition time for Xray fluorescence imaging of gold nanoparticles: a preliminary study using photon counting detector Biophotonics and Immune Responses Xi 9709

Seller P, Bell S, Cernik R J, Christodoulou C, Egan C K, Gaskin J A, Jacques S, Pani S, Ramsey B D, Reid C, Sellin P J, Scuffham J W, Speller R D, Wilson M D and Veale M C 2011 Pixellated Cd(Zn)Te high-energy X-ray instrument Journal of Instrumentation 6 C12009C

von Busch H, Harding G, Martens G, Schlomka J P and Schweizer B 2005 Investigation of externally activated x-ray fluorescence tomography for use in medical diagnostics Proceedings of SPIE $\mathbf{5 7 4 5} 90$

Yoon C, Kim Y and Lee W 2016 3D Non-Destructive Fluorescent X-Ray Computed Tomography With a CdTe Array IEEE Trans. Nucl. Sci. 63 1844-53

Zhang S Y, Li L, Chen J Y, Chen Z Q, Zhang W L and Lu H B 2019 Quantitative Imaging of Gd Nanoparticles in Mice Using Benchtop Cone-Beam X-ray Fluorescence Computed Tomography System Int. J. Mol. Sci. 20 


\title{
Micro- and Nanodosimetry of MNPs
}

\author{
Hans Rabus ${ }^{1}$, Elisabetta Gargioni ${ }^{2}$ \\ 1 Physikalisch-Technische Bundesanstalt (PTB), 38116 Braunschweig, Germany \\ 2 Department of Radiotherapy and Radiation Oncology, University medical center Hamburg-Eppendorf, 20246 \\ Hamburg, Germany
}

\section{Status}

In irradiated biological cells containing metallic nanoparticles (MNPs), the presence of the MNPs leads to a local enhancement of the radiation interaction probability. Generally, the atoms in the MNP have a higher atomic number $(Z)$ than the atoms constituting biomolecules and water. Hence, they have higher interaction cross sections. Furthermore, radiation interaction with the MNPs not only leads to the production of additional secondary electrons but also to the occurrence of Auger cascades. Auger electrons with energies in the range of few keV deposit most of their energy in the proximity of the MNPs. This implies, in principle, that steep energy deposition gradients may occur as well as short-range changes of the radiation quality (particle track structure) on the nanometer scale (McMahon et al 2011).

So far, the investigation of these physical radiation effects of MNPs has only been possible by numerical

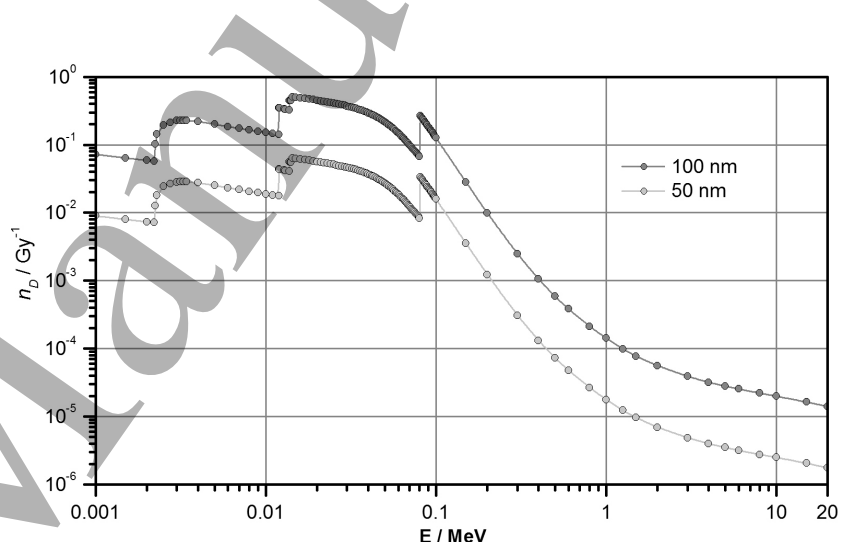
simulations using Monte Carlo codes. Until recently, many common Monte Carlo codes did not allow track

Figure 1. Photon energy dependence of the number of photon interactions per absorbed dose in spherical gold nanoparticles of $50 \mathrm{~nm}$ and $100 \mathrm{~nm}$ diameter. structure calculation. Therefore, most published studies have focused on the calculation of the enhancement of the absorbed dose around the MNP, where results may differ by several orders of magnitude among the published studies even for similar simulated setups(Li et al 2020). Only few simulation studies have investigated other quantities like the radiation damage due to low-energy electrons which lead to clustered ionizations at the nanometric scale (Gargioni et al 2016, Dressel et al 2017, 2019).

\section{Current and future challenges}

Simulations of microdosimetric and nanodosimetric effects of MNPs face the same computational challenges as the host of simulations are aimed at determining the local enhancement of absorbed dose. The most important of these challenges is related to the small dimensions of the nanoparticles which imply a small probability for individual particle tracks or trajectories to intersect with the nanoparticle volume. Therefore, realistic simulations of physical radiation effects require the CPU-intense simulation of large numbers of primary particle histories or the application of suitable variance reduction techniques. However, seemingly 
straightforward solutions such as reducing the incident field size may lead to simulation scenarios where the results are strongly biased by a lack of secondary particle equilibrium (examples can be found in (Li et al 2020, Dressel et al 2017)). For simulations of dose enhancement by MNPs in photon fields, the lack of contribution of energy depositions from secondary particles that would be produced in an extended radiation field by photon interactions outside the confined region of the simulated primary photon field, can be estimated from literature data of photon interaction cross sections (Rabus et al 2019). For track structure simulations, a corresponding correction approach may also exist but has not yet been identified. The multiscale nature of the problem means that there is a need to consider energy deposition within micro/nano-scopic dimensions across macroscopic length scales (Zygmanski and Sajo 2016, Martinov and Thomson 2016).

Another computational challenge for micro- and nanodosimetry of MNPs is related to the fact that in addition to the stochastics of the electron tracks emerging from the MNPs, an additional stochastic factor comes into play which is related to the probability that an MNP in a biological cell interacts with the incident radiation. Figure 1 shows the photon energy dependence of the probability per dose that a single gold MNP of $100 \mathrm{~nm}$ and $50 \mathrm{~nm}$ diameter will experience a photon interaction. (This probability scales with the third power of the MNP diameter.) It is quite evident that for realistic values of absorbed dose the probability is generally significantly lower than unity such that the effect of the Poisson statistics of the photon interactions with the MNPs needs to be considered.

For microdosimetric computations this may not be an issue if the considered targets (cell nucleus or chromosome domains) are large enough to contain a large number of MNPs. For smaller target sizes in the nanometer range and for nanodosimetry, on the other hand, there may be a need to focus on conditional specific energy distributions and cluster size distributions for the vicinity of particular MNPs (that experienced a photon interaction).

In addition to these methodological issues, there, is also the general challenge of computational micro- and nanodosimetry related to the different cross section models used by different codes and to potential additional interaction channels, such as collective excitations within the MNP (Verkhovtsev et al 2014). Different cross section models have been shown to lead to drastic differences particularly between results for nanodosimetric quantities (Villagrasa et al 2019). Additional quantum mechanical effects such as the Heisenberg uncertainty principle further challenge the accuracy at short length scales and very low energies (Thomson and Kawrakow 2011, Liljequist and Nikjoo 2014). A proper assessment of the uncertainties of such simulation results probably cannot be only based on comparison of computational approaches. It will rather be necessary to develop and perform benchmark experiments that test the predictions of the codes. A more long-term challenge that goes even further would be the development of experimental approaches measuring nanodosimetric cluster size distributions in the presence of MNPs directly.

\section{Advances in science and technology to meet the challenges}

Several groups around the world are working on improving the cross-section data used in simulation codes for applications involving nanoparticles (Wälzlein et al 2014). Some of the augmented data sets include effects like 
changes in the surface plasmons that were predicted theoretically (Verkhovtsev et al 2014). For the case of interactions of MNP with protons, experimental validation of the cross sections by comparison to direct measurements of the yield of emitted electrons has already been performed (Hespeels et al 2019). For photon irradiation, similar studies are in progress. Such experiments require a careful consideration of the stochastic aspects of the radiation interaction with MNPs.

Direct measurement of the influence of MNPs on microdosimetric spectra have been proposed using avalancheconfined tissue-equivalent proportional counters that are capable of simulating nanometric site sizes (Mazzucconi et al 2020). The idea is to build such detectors with an exchangeable cylindrical outer electrode and to use electrodes coated with MNP on the inner surface or incorporating MNPs in the material of the electrodes.

Figure 2 shows illustrations of two other approaches to directly measure the enhancement of radiation effects by gold nanoparticles on DNA-based detector structures. The first approach is based on the so-called DNA-dosimeter concept where DNA strands are used as resistive elements in nano-circuits and strand breaks are detected by changes in the electrical conductivity (Heimbach et al 2017). In contrast to this early work, DNA scaffolds would be used to add MNPs in defined positions from the DNA conductors to study the change in yield of radiation-induced strand breaks in the presence of MNP in functional dependence on their distance from the target.

The second approach illustrated in the lower panel of Figure 2 would use the DNA origami folding technique to produce a base from which oligonucleotides are protruding that are terminated by larrge molecules,
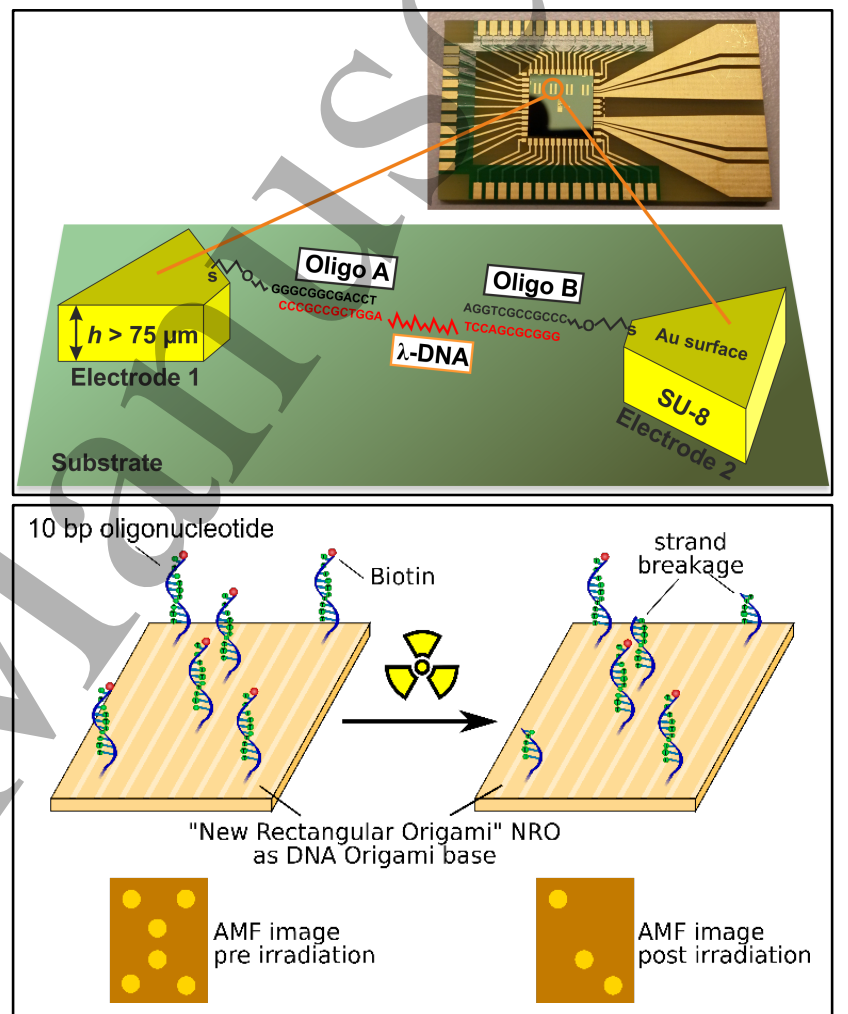

Figure 2. Illustrations of two proposed experimental approaches for measuring the nanodosimetric effects of gold MNPs on DNA-based structures. For details see text. such as biotin. These biotin molecules can be detected by atomic force microscopy (AFM). Here, radiation damage in form of DNA strand breaks would be detected (in a destructive manner) by the disappearance of object spots in the AFM images.

The influence of MNPs would be studied in the same way as before, i.e. by comparing test structures without nanoparticles with structures where MNPs are incorporated at defined positions. The advantage of the second approach would be that measurements can in principle also be performed in a liquid-water environment to also study the influence of MNPs on the production of free radical species. 


\section{Concluding remarks}

The envisioned experiments described above will not only be useful for validating simulations of the physical microdosimetric and nanodosimetric effects of MNPs but also, in the long run, such experiments may be further developed to provide a comprehensive set of benchmark data for the complete simulation chain, including the physical, physico-chemical, and chemical stages. This would allow fixing some of the simulation parameters such as, for instance, the contribution of molecular excitation by very low energy electrons to direct strand breaks or the probability of breaking the backbone chain by reactions with hydroxyl radicals. In this way, they may contribute to a synoptic picture of the radiation effects of MNPs that encompasses their physical, chemical and physico-chemical contribution to the radiobiological enhancement of irradiation on the micrometer and nanometer scale.

\section{Acknowledgments}

We acknowledge Woon Yong Baek from PTB and Jussi Toppari from Jyväskylä University (Finland) for contributing their ideas for measurement of radiation effects of nanoparticles using DNA origami structures, Valeria Conte from INFN (Italy) for sharing her ideas regarding microdosimetry of nanoparticles, and Heike Nittmann for preparing the respective illustrations.

\section{References}

Dressel T, Bug M and Gargioni E 2017 Monte-Carlo calculated energy deposition and nanodosimetric quantities around a gold nanoparticle Radiotherapy and Oncology 123 S421

Dressel T, Bug M U, Gargioni E and Rabus H 2019 An algorithm to Determine the Nanodosimetric Impact of Gold Nanoparticles on Cell Models. Radiation Protection Dosimetry 183 55-9

Gargioni E, Dressel T, Rabus H and Bug M U 2016 Monte-Carlo calculation of the secondary electron spectra inside and around gold nanoparticles Radiotherapy and Oncology 119 S926

Heimbach F, Arndt A, Nettelbeck H, Langner F, Giesen U, Rabus H, Sellner S, Toppari J, Shen B and Baek W Y 2017 Measurement of changes in impedance of DNA nanowires due to radiation induced structural damage Eur. Phys. J.D 71211

Hespeels F, Heuskin A C, Tabarrant T, Scifoni E, Kraemer M, Chêne G, Strivay D and Lucas S 2019 Backscattered electron emission after proton impact on gold nanoparticles with and without polymer shell coating. Phys. Med. Biol. 64125007

Li W B, Belchior A, Beuve M, Chen Y Z, Di Maria S, Friedland W, Gervais B, Heide B, Hocine N, Ipatov A, Klapproth A P, Li C Y, Li J L, Multhoff G, Poignant F, Qiu R, Rabus H, Rudek B, Schuemann J, Stangl S, Testa E, Villagrasa C, Xie W Z and Zhang Y B 2020 Intercomparison of dose enhancement ratio and secondary electron spectra for gold nanoparticles irradiated by X-rays calculated using multiple Monte Carlo simulation codes. Phys Med 69 147-63 
Liljequist D and Nikjoo H 2014 On the validity of trajectory methods for calculating the transport of very low energy ( $<1 \mathrm{keV})$ electrons in liquids and amorphous media Radiat. Phys. Chem. 99 45-52

Martinov M P and Thomson R M 2017 Heterogeneous multiscale Monte Carlo simulations for gold nanoparticle radiosensitization. Med. Phys. 44 644-53

Mazzucconi D, Bortot D, Martin Rodriguez P, Pola A, Fazzi A, Colautti P, Conte V, Selva, and Agosteo S 2020 A wall-less Tissue Equivalent Proportional Counter as connecting bridge from microdosimetry to nanodosimetry. Radiat. Phys. Chem. 171108729

McMahon S J, Hyland W B, Muir M F, Coulter J A, Jain S, Butterworth K T, Schettino G, Dickson G R, Hounsell A R, O'Sullivan J M, Prise K M, Hirst D G and Currell F J 2011 Biological consequences of nanoscale energy deposition near irradiated heavy atom nanoparticles. Sci Rep 118

Rabus H, Gargioni E, Li W B, Nettelbeck H and Villagrasa C 2019 Determining dose enhancement factors of high-Z nanoparticles from simulations where lateral secondary particle disequilibrium exists. Phys. Med. Biol. 64155016

Thomson R M and Kawrakow I 2011 On the Monte Carlo simulation of electron transport in the sub-1 keV energy range. Med. Phys. 38 4531-4

Verkhovtsev A V, Korol A V and Solov'yov A V 2014 Revealing the Mechanism of Low-Energy Electron Yield Enhancement from Sensitizing Nanoparticles Phys. Rev. Lett. 114063401

Villagrasa C, Bordage M C, Bueno M, Bug M, Chiriotti S, Gargioni E, Heide B, Nettelbeck H, Parisi A and Rabus H 2019 Assessing the Contribution of Cross-section to the Uncertainty of Monte Carlo Calculations in Micro- and Nanodosimetry Radiation Protection Dosimetry 183 11-6

Wälzlein C, Scifoni E, Krämer M and Durante M 2014 Simulations of dose enhancement for heavy atom nanoparticles irradiated by protons. Phys. Med. Biol. 59 1441-58

Zygmanski P and Sajo E 2016 Nanoscale radiation transport and clinical beam modeling for gold nanoparticle dose enhanced radiotherapy (GNPT) using X-rays. Br J Radiol 8920150200 


\title{
Can Physics Explain the Radiation Enhancement Effects of MNPs?
}

\author{
Anne-Catherine Heuskin ${ }^{1}$, Sébastien Penninckx ${ }^{1}$, Félicien Hespeels ${ }^{1}$, Stéphane Lucas ${ }^{1}$ \\ ${ }^{1}$ NAmur Research Institute for LIfe Sciences (NARILIS), Laboratory of Analysis by Nuclear Reactions, University of Namur, 61
} rue de Bruxelles, 5000 Namur, Belgium.

\section{Status}

When localized in the vicinity of tumor cells, high Z metallic nanoparticles are thought to enhance energy deposition upon radiation treatment. One has first to distinguish between radiation types. keV photons often used in radiobiology studies cause the strongest effect: in this energy range, most of the interactions with the surrounding media occur via the photoelectric effect (PE) which is proportional to $Z^{3} / E^{3}$. Accordingly, impinging photons trigger more interactions in metal material than in the surrounding water. The effect is further reinforced through the deexcitation cascade: the created vacancy will be filled by outer shell electrons, leading to the emission of characteristic X-rays or low energy Auger electrons. The combination of photoelectrons, Auger electrons and to a lesser extent characteristic X-rays is believed to add extra dose outside MNPs [1]. The effect is spread at the nanometer level (as the range of Auger electrons is limited to $<100 \mathrm{~nm}$ ) and extends micrometers away from MNP due to the other secondaries which typically have energies close to the primary photons. For instance, the gold K-edge at $80.7 \mathrm{keV}$ adds a boost in the PE coefficient if the primaries energy is chosen accordingly. However, in the MeV range, the mass absorption coefficient for water and gold are similar. The probability to enhance the deposited dose is then reduced and limited to the cascade effect upon gold ionization. The combination of photon radiation and MNP thus results in local radiation hardening and pushes the modality toward a high linear energy transfer (LET) type.

The PE mechanism explained above is often put forward to justify the observed enhancement after Xray irradiation. Indeed, the ratio of the mass attenuation coefficient of gold to water can reach 160 near the gold K-edge [2]. However, a number of experimental studies reported significant radiosensitization effects when gold nanoparticles (GNPs) were used at concentrations as low as 0.003 $\%$ of gold per mass of tissue (weight percent, WP) [3]. Most simulation studies report predictions using typical 0.1 to $1 \mathrm{WP}$, an overrated amount when considering the possible transition to human medicine. According to this amount, it is possible to derive a more realistic mass attenuation coefficient for a homogeneous mixture of water and gold. 


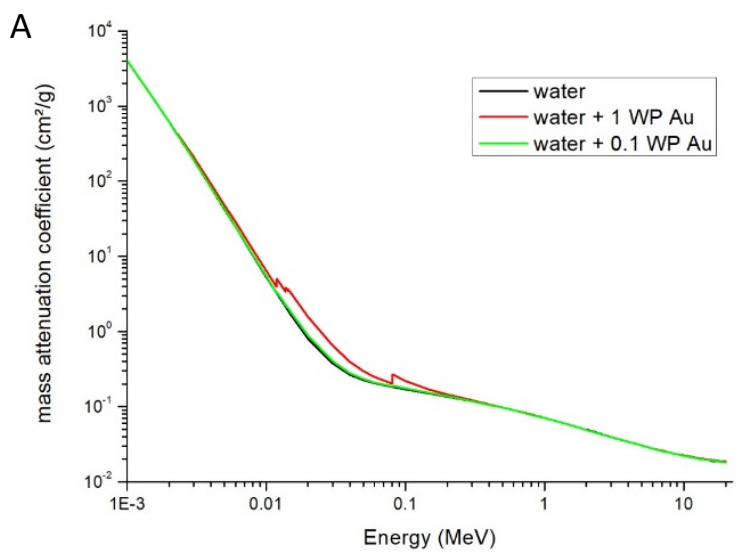

B

Figure 1 (A) Mass attenuation coefficient for water (black), water with 1\% gold mass (red) and water with $0.1 \%$ gold mass (green) (B) Mass attenuation coefficient for water (black), water with $1 \%$ gold mass (red) and corresponding ratio according to energy (blue)

Figure $1 \mathrm{~A}$ displays the mass attenuation coefficients with 1 and $0.1 \%$ gold mass. 0.1 WP is not sufficient to produce a significant change in the mass attenuation coefficient. However, the $K$ and $L$ edges of gold are discernible if the concentration is increased to 1 WP. The corresponding ratio is presented in Figure $1 \mathrm{~B}$, with a strong peak in the keV range as expected, although with a maximum twofold increase.

In addition to limits in the gold amount uptake in cells, it has to be noted that the probability for a GNP to incur PE will not only depend on the gold mass attenuation coefficient but also on its size, with the smallest being the worst case. Lin et al. simulated the number of electrons produced through photon interaction with a directly targeted $50 \mathrm{~nm}$ GNP and reported values in the $10^{-5}-10^{-4}$ range per incoming photon at best [4]. A similar finding was highlighted in a simulation study of McMahon et al. [5]. Over $99 \%$ of GNP present in a cell do not contribute to the dose enhancement effect, even if the few incurring ionizations produce dramatic spikes in dose.

In the past decade, proton therapy centers flourished around the world and the question of dose enhancement thanks to GNP was raised again. The radioenhancement mechanism provided by GNPs originate from mere gold ionizations (with associated cascade), the stopping power in gold being greater by a factor from 2 to 10 compared to water. However, the qualities of generated electrons differ according to the energy spectra of incoming protons. The maximum energy transferred to an electron at rest is given by $Q_{\max }=\frac{2 \gamma^{2} m V^{2}}{1+\frac{2 \gamma m}{M}+\frac{m^{2}}{M^{2}}}$, with $M$ and $V$ the mass and the velocity of the proton, respectively, $m$ the mass of the electron and $\gamma$ the relativistic factor. In patients, most created electrons have energies in the range of hundreds of keV near the proximal end of the tumor while rare 


\section{Current and future challenges}

In general, biologically observed enhancement values are higher than predicted [10].

However, Monte Carlo codes used to compute these predictions often rely on incomplete Auger cascades. For instance, the deexcitation module after proton ionization in Geant4 is only available up to the $\mathrm{M}$ shell. Vacancies created in outer shells are thus not followed and the potential increase in dose is not taken into account. This is not a major issue for photon irradiation simulations. However, medium and high LET protons are unable to ionize inner shells as they deliver a few keV at most and most ionizations will take place in the outer shells. Moreover, Auger relaxation is the most probable in this case, as its probability increases with increasing shell number (fluorescence yield $=0.0245$ for the $\mathrm{M}$ shell in gold). It is thus very likely that proton simulations performed with Geant4 so far underestimated the yield of Auger electrons for high Z targets.

In addition, some codes, like Geant4, rely on condensed history models. These models are more adapted for macroscopic volumes and electron tracking for energies above a few hundred keV. Geant4 models G4BetheBlochModel and G4BraggModel do not take into account the generation of electrons below the mean ionization potential of the medium (790 eV for gold) which leads to an underestimation of low energy electron yield. This underestimation has been recently highlighted by comparison with experimental results for proton impact on gold nanoparticles. To reproduce low energy electron emission spectra from a gold target, a single interaction approach, like the one used in TRAX, NOREC and Geant4-DNA, should be preferred $[11,12]$. 


\section{Advances in science and technology to meet challenges}

The reliability of track structure codes depends on the implemented interaction cross section sets which are not easily accessible for low energy projectiles. Validation for the TRAX cross sections has been performed indirectly by reproducing experimental data of backscattered electron emission from gold film and GNPs [13]. Since the electron energy distribution is anisotropic, data are still needed in the forward direction where a larger amount of high energy electrons is expected.

As pointed out in numerous experimental works, the spatial distribution of GNPs in célls is not homogenous as assumed in many simulation studies. Aggregation of GNPs is often observed and could decrease radioenhancement by trapping produced electrons hence adding a buffering effect. Accurate representations such as GNP packing in vesicles distributed in cytoplasm should be preferred [14]. In addition, there is a crucial need for the modelling of more realistic geometries at the cell level, with a detailed description of components such as organelles or DNA structure. TOPAS-nBio was recently released to help the understanding of radiobiological processes at the cell and sub-cellular levels, also including a large set of chemical reactions [15]. The inclusion of the cytosol scavenging properties would represent another step forward.

Current findings also raise questions concerning the radiosensitization mechanism which do not seem to be solely driven by physical effects. The great disparity between physical dose enhancement predictions and the experimentally observed radiosensitization effects suggests a biological component to the radiosensitization mechanism. Increasing evidences supporting this concept are piling up highlighting the key role played by the oxidative stress in the radioenhancement effect. In recent articles, Daems et al. and Penninckx et al. demonstrated that gold nanoparticles have the ability to inhibit thioredoxin reductase (TrxR) and gluthatione reductase, regulators of redox reactions, in cancer and normal cells [16-18]. TrxR inhibition induces a cell weakening effect prior to radiation exposure, which if timed wisely can lead to a radiosensitization effect. In the context of personalized medicine, genomic assays for mRNA TXNRD1 expression in tumor biopsies can thus drive the application of GNP radiosensitization in the clinic.

\section{Concluding remarks}

The preceding discussion points out that although there is a moderate radioenhancing effect demonstrated for keV photons, additional dose deposited when using MeV photons or protons is limited. The development of single interaction Monte Carlo simulations and accurate geometries should shed light on this matter. However, the biological component seems preponderant in the MNP 
action mechanism. More studies are needed to assess the impact of MNP on biological pathways in the absence of radiation, such as regulation of genes involved in DNA repair, slowing down of cell proliferation or disruption of the cellular redox balance. The scientific community should thus reconsider the original theory of physical enhancement and encompass the role of MNPs on decreased detoxification potential of cells.

\section{References}

1. Carter, J.D., et al., Nanoscale energy deposition by X-ray absorbing nanostructures. J Phys Chem B, 2007. 111(40): p. 11622-5.

2. Mesbahi, A., A review on gold nanoparticles radiosensitization effect in radiation therapy of cancer. Rep Pract Oncol Radiother, 2010. 15(6): p. 176-80.

3. Liu, X., et al., The synergistic radiosensitizing effect of tirapazamine-conjugated gold nanoparticles on human hepatoma HepG2 cells under X-ray irradiation. Int J Nanomedicine, 2016. 11: p. 3517-31.

4. Lin, Y., et al., Comparing gold nano-particle enhanced radiotherapy with protons, megavoltage photons and kilovoltage photons: a Monte Carlo simulation. Phys Med Biol, 2014. 59(24): p. 7675-89.

5. McMahon, S.J., et al., Biological consequences of nanoscale energy deposition near irradiated heavy atom nanoparticles. Sci Rep, 2011. 1: p. 18.

6. Heuskin, A.C., et al., Metallic nanoparticles irradiated by low-energy protons for radiation therapy: Are there significant physical effects to enhance the dose delivery? Med Phys, 2017. 44(8): p. 4299-4312.

7. Jeynes, J.C., et al., Investigation of gold nanoparticle radiosensitization mechanisms using a free radical scavenger and protons of different energies. Phys Med Biol, 2014. 59(21): p. 6431-43.

8. Lin, Y., et al., Gold nanoparticle induced vasculature damage in radiotherapy: Comparing protons, megavoltage photons, and kilovoltage photons. Med Phys, 2015. 42(10): p. 5890902.

9. Lin, Y., et al., Biological modeling of gold nanoparticle enhanced radiotherapy for proton therapy. Phys Med Biol, 2015. 60(10): p. 4149-68.

10. Butterworth, K.T., et al., Physical basis and biological mechanisms of gold nanoparticle radiosensitization. Nanoscale, 2012. 4(16): p. 4830-8. 
11. Hespeels, F., et al., Experimental measurements validate the use of the binary encounter approximation model to accurately compute proton induced dose and radiolysis enhancement from gold nanoparticles. Phys Med Biol, 2019. 64(6): p. 065014.

12. Walzlein, C., et al., Simulations of dose enhancement for heavy atom nanoparticles irradiated by protons. Phys Med Biol, 2014. 59(6): p. 1441-58.

13. Hespeels, F., et al., Backscattered electron emission after proton impact on gold nanoparticles with and without polymer shell coating. Phys Med Biol, 2019.

14. Sotiropoulos, M., et al., Modelling direct DNA damage for gold nanoparticle enhanced proton therapy. Nanoscale, 2017. 9(46): p. 18413-18422.

15. Schuemann, J., et al., TOPAS-nBio: An Extension to the TOPAS Simulation Toolkit for Cellular and Sub-cellular Radiobiology. Radiat Res, 2019. 191(2): p. 125-138.

16. Penninckx, S., et al., The role of thioredoxin reductase in gold nanoparticle radiosensitization effects. Nanomedicine (Lond), 2018. 13(22): p. 2917-2937.

17. Penninckx, S., et al., Thioredoxin Reductase Activity Predicts Gold Nanoparticle Radiosensitization Effect. Nanomaterials (Basel), 2019. 9(2).

18. Daems, N., et al., Gold nanoparticles affect the antioxidant status in selected normal human cells. Int J Nanomedicine, 2019. 14: p. 4991-5015. 


\title{
Role of Low Energy Electrons in Local MNP Radiation Enhancement
}

\author{
Léon Sanche ${ }^{1}$ \\ ${ }^{1}$ Department of nuclear medicine and radiobiology, Université de Sherbrooke, \\ Sherbrooke, Canada \\ E-mail: leon.sanche@usherbrooke.ca
}

\section{Status}

It is well known that electrons are emitted from the impact of energetic particles or photons on a metal surface. A similar behavior is expected from a metal nanoparticle (MNP) $[1,2]$. For photons, the mass-energy absorption coefficient is larger by roughly two orders of magnitude in the $10-80 \mathrm{keV}$ range, compared to that of biological tissue [3]. The yield of emitted electrons, which extends from a few eV to energies close to the primary photon energy [2], is proportionally increased in magnitude. However, the electron energy distribution depends not only on the material, but also on the size and shape of the MNP. Electrons emitted with energies above the ionization potential of the medium produce a further generation of lower energy electrons. For initial charged particles, the secondary electron distribution lies essentially in the low-energy (0-30 eV) range due to their production via the excitation of plasmons in the MNP [4]. According to recent calculations, the number of these low energy electrons (LEEs, $<30 \mathrm{eV}$ ) produced by, high-energy protons interacting with a gold NP (GNPs) would be about an order of magnitude higher than that from an equivalent water volume and not very dependent on the primary particle energy [4]. In contrast, the high electron-emission efficiency of X-ray-irradiated MNPs is restricted to initial energies below $300 \mathrm{keV}$ [3].

Considering the range of electrons in water [5] and the spherical geometrical factor, most of the LEE density lies within submicrometer distances from the surface of a MNP irradiated with fast charged particles or $10-80 \mathrm{keV}$ photons $[1,2]$. These LEEs are the most numerous reactive species created around the MNP and carry a large portion of the energy absorbed by the metal. They strongly react with the surrounding medium, mainly via a resonant interaction, which leads to their temporary capture by molecules or moieties of large biomolecules. This capture leads to the formation of molecular transient anions (TA) that decay by reemitting the captured electron (i.e., autoionization) or by dissociating (i.e., dissociative electron attachment) [6]. Since autoionization can leave the target 
molecule in a dissociative electronic state, both channels can break bonds and damage biomolecules [6]. However, in complex molecules, such as DNA, electron transfer between basic units increases the complexity of the damage mechanism [7].

During the last two decades, there have been numerous experimental and theoretical studies on the interaction of LEEs with gaseous or complex condensed biomolecules, ranging from amino acids, peptides, DNA bases, sugar and phosphate groups, to oligonucleotides of short DNA strands and bacterial DNA [6-10]. The condensed-phase targets usually consisted of self-assembled monolayers and multi- or sub-monolayer films of the biomolecule deposited on a conductive substrate. The type of LEE-induced damages in DNA, analyzed by various techniques, included single and double strand breaks, base damage and cleavage, and clustered lesions, consisting of single strand breaks and base damages $[6,7,10]$. From yields vs energy curves, it was generally concluded that the decay of TAs into destructive channels played a major role in inducing these lesions [6-9].

Fig. 1 shows damage enhancement factors (EFs) resulting from irradiation of DNA-GNP complexes with 60-keV electrons. In these experiments, 5-nm diameter GNPs were bound in ratio 1:1 to plasmid DNA [10]. For production of a potentially lethal lesion, such as a DSB, bare GNPs produce an EF of about 2.3, as shown by the left column (blue) of the middle group in Fig. 1. With GNPs coated with ligands of 2.5 to $4 \mathrm{~nm}$, the corresponding EFs for DSBs are reduced to 1.5 (green column) and 1.0 (red), respectively. This result corroborates theoretical predictions on the electron distributions from fast charged particles [4], as it implies that energetic electrons interacting with MNPs generate essentially LEEs of extremely short ranges $(<10 \mathrm{~nm})$. When a similar experiment is performed with 200-keV X-ray irradiation of 5-30 nm diameter PEG-coated GNPs, longer ligands ( $\sim 11 \mathrm{~nm}$ ) are needed to obtain only a $60 \%$ reduction in EF [11], as expected from the larger thermalization range of the X-ray photoelectrons $[2,4,5]$.

\section{Current and future challenges:}

Despite the numerous publications that have appeared in the last decade on MNPs, we still don't have any experimental results showing the actual distribution of electrons emitted from isolated MNPs of different sizes, interacting with various types of high-energy ionizing radiation. The present electron distributions rely on theoretical and model calculations [1,2, 4]. Many parameters need to be included and refined in these calculations, so that, both at the experimental and theoretical level, the generation of accurate electron distributions in biological media remains a challenge.

The efficient use of MNPs in Radiotherapy is limited by the nanoscale range of most emitted electrons and their secondaries, unless the MNPs can be targeted to vital cellular molecules, such as DNA. Otherwise, the MNPs only serve to increase the energy deposited and radicals randomly created 


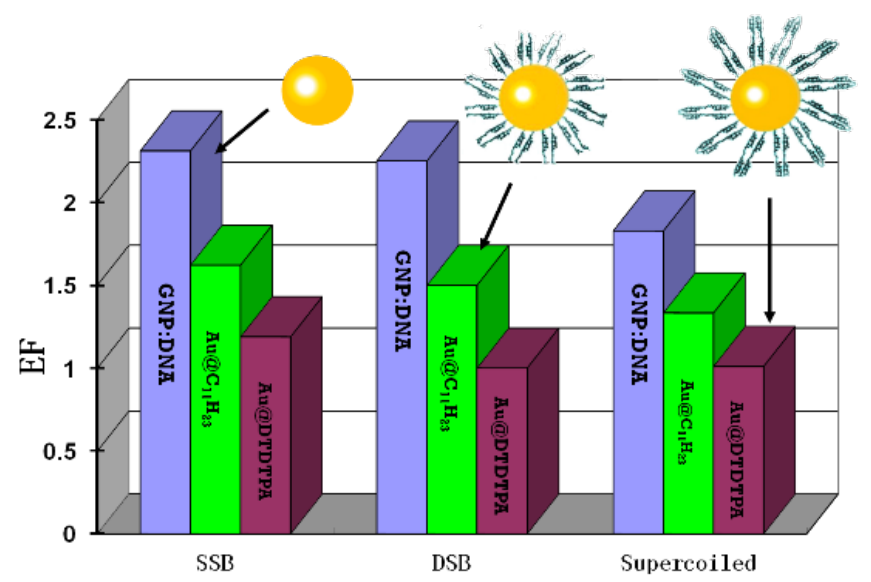

Figure 1. EFs for the formation of SSBS, DSBs and loss of initial DNA configuration induced by $60 \mathrm{keV}$ electrons in GNP-DNA complexes with ratio 1:1. In each group of three histograms, the EFs correspond to enhancement factors for a specific DNA damage, when GNPs of $5 \mathrm{~nm}$ diameter bound to DNA are bare or coated with $\mathrm{C}_{11} \mathrm{H}_{23}$ or DTDTPA (i.e., dithiolated diethylene triaminepentaacetic) ligands.

in the cytoplasm or outside the cell. Moreover, to take advantage of the high electron-emission coefficient of $10-80 \mathrm{keV}$ photons, conventional X-ray Radiotherapy must be limited to superficial tumors.

\section{Advances in science and technology to meet the challenges}

Electron-energy distributions emitted from irradiated gaseous MNPs could be measured using highresolution electron energy analysers. Photoemission spectra measurements of free core-shell nanoparticles [12] and X-ray photoelectron spectroscopy of isolated NP beams [13], which already exist to investigate X-ray photoelectron emission from non-metallic NPs of different sizes, could possibly be adapted to vaporized MNPs. On the other hand, the present distributions could be improved by more elaborate calculations of (1) the slowing down of electrons within the MNP, particularly in the low-energy regime; (2) the number of electrons emitted per initial interaction, (3) the number of electrons that recombine with the MNP and (4) the interaction with the MNP of electrons backscattered around and into the nanospheres. In such computations in biological media, it would be advantageous to consider the electrostatic potential created between the MNP and the escaping electron, including the charge-induced polarization of the medium and the multiple positive charges created in the MNP, which in turn influence the number and energy of emitted electrons.

Targeting of specific components of cancer cells (e.g., the nucleus) is possible with vector molecules that bind to MNPs $[14,15]$. However, for maximum efficiency, the MNP should be either coated with the shortest possible ligand, or be only partially coated, to reduce as much as possible, attenuation of 
the short-range highly-destructive LEEs. Finally, it should be noted that efficient X-ray therapy need not be limited to superficial tumors, if the MNP is accompanied by a therapeutic radioisotope. In other words, MNPs could improve localization of radiation energy in targeted radionuclide therapy (TRT) $[15,17,19]$, where the emitted short-range particles are often accompanied by the emission of longerrange photons [16] that could be partially absorbed by the metal and produce LEEs. GNPs have already been complexed with chelators that bind to Auger-electron and beta-particle emitters used in IRT $[15,17]$. Encapsulation of such radionuclides in gold nanocages was also suggested to convert into LEES particle radiation and the accompanying photon flux, which reaches healthy tissues during TRT [18].

\section{Concluding remarks and acknowledgments}

Considering future quantification of the species produced around irradiated MNPs and ongoing refinement in the methods of targeting them to cancer cells, via intravenous administration of carriers $[15,19,20]$ or direct intratumoral injection $[21,22]$, such particles should become highly efficient for treating tumors and their metastasis. These methods are also promising for extending the field of radiobiology to the nanoscopic level.

\section{Acknowledgements}

Financial support was provided by the Canadian Institute of Health Research (PJT-162325). The author is indebted to Dr. Fred Currell, Yi Zheng, Balder Villagomez and Andrew Bass for informative discussions.

\section{References}

[1] Wardlow N, Poulin C, Villagomez-Bernabe B and Currell F 2015 A simple model to quantify radiolytic production following electron emission from heavy-atom nanoparticles irradiated in liquid suspensions Radiat Res. 154 518-532

[2] McQuaid HN, Muir MF, Taggart LE, McMahon SJ, Cloutier JA, Hyland WB, Jain S, Betterworth KT, Schettino G, Prise KM, Hirst D, Botchway SW and Currell F 2016 Imaging and radiation effects of gold nanoparticles in tumour cells Sci. Rep. 6 19442-199452 and citations therein.

[3] Hubbell J, Seltzer S 1996 Table of $x$-ray mass attenuation and mass energy absorption coefficients NIST Report 
[4] Verkhovtsev AV, Korol AV and Solov'yov AV 2015 Electron Production by Sensitizing Gold Nanoparticles Irradiated by Fast lons J. Phys. C 119 11000-11013

[5] Meesungnoen J, Jay-Gerin JP, Filali-Mouhim A and Mankhetkorn S 2002 Low energy electron penetration range in liquid water Radia Res. 158 657-60

[6] Sanche L 2009 Low Energy Electron Interaction with DNA: Dissociation and Formation of Transient Anions, Radicals and Radical Anions Radical and Radical Ion Reactivity in Nucleic-Acid Chemistry ed Wiley-Blackwell 239-93

[7] Shao Y, Dong Y, Hunting D, Zheng Y and Sanche L 2017 Unified Mechanism for the Generation of Isolated and Clustered DNA Damages by a Single Low Energy (5-10 eV) Electron J. Phys. Chem. C 121(4) $2466-72$

[8] Baccarelli I, Bald I, Gianturco FA, Illenberger E and Kopyra J 2011 Electron-induced damage of DNA and its components: Experiments and theoretical models Phys. Rep. 508(1) 1-44

[9] Rackwitz J, Kopyra J, Dabkowska I, Ebel K, Rankovic M, Milosavljevic AR and Bald I 2016 Sensitizing DNA Towards Low-Energy Electrons with 2-Fluoroadenine Angew. Chem. Int. Edit. 55(35) 10248-52 and citations therein

[10] Zheng Y and Sanche L 2013 Low energy electrons in nanoscale, radiation physics: relationship to radiosensitization and chemoradiation therapy. Rev. Nanoscience Nanotechnology 2 1-28

[11] Spaas C, Dok R, Deschaume O, De Roo B, Vervaele M, Seo JW, Bartic C, Hoet P, Van den Heuvel F, Nuyts S and Locquet JP 2016 Dependence of gold nanoparticle radiosensitization on functionalizing layer thickness Radiat Res 185(4) 384-392

[12] Jacobs MI, Kostko MA and Wilson KR 2017 Low energy electron attenuation lengths in core-shell nanoparticles Phys. Chem. Chem. Phys. 19 13372-13378

[13] Sublemontier O, Nicolas, C, Aureau D, Patanen M, Kintz H, Liu X, Gaveau MA, Le Garrec JL, Robert E, Barreda FA, Etcheberry A, Reynaud C, Mitchel JB and Miron C 2014 X-ray Photoelectron Spectroscopy of Isolated Nanoparticles J. Phys. Chem. Lett. 5(19) 3399-3403

[14] Yang C and Chithrani D. 2016. Nuclear targeting of gold nanoparticles for improved therapeutics. Curr Top Med Chem. 16 271-280

[15] Yook S, Cai Z, Lu Y, Winnik MA, Pignol JP and Reilly RM 2015 Radiation Nanomedicine for EGRFPositive Breast Cancer: Punitumumab-Modified Gold Nanoparticles Complexed to the $\beta$-ParticleEmitter Mol Pharm. 2015 Nov 2; 12(11) 3963-72

[16] Handbook of Nuclear Chemistry 2nd ed. A. Vertes, et al., (Springer, 2011)

[17] Cai Z, Chattopadhyay N, Yang K, Kwon YL, Yook S, Pignol JP, and Reilly RM 2016111 In-labeled trastuzumab-modified gold nanoparticles are cytotoxic in vitro to HER2-positive breast cancer 
cells and arrest tumor growth in vivo in athymic mice after intratumoral injection Nucl. Med. and Biol. 43(12) 818-26.

[18] Sanche L 2015. Cancer treatment: low-energy electron therapy Nat. Mater. 14 861-863

[19] Brazzale C, Mastrotto F, Moody P, Watson PD, Balasso A, Malfanti A, Mantovani G, Caliceti P, Alexander C, Jones AT and Salmaso S. 2017. Control of targeting ligand display by pH-responsive polymers on gold nanoparticles mediates selective entry into cancer cells Nanoscale 9(31) 11137-11147

[20] Ngwa W, Kumar R, Sridhar S, Korideck H, Zygmanski P, Cormack RA, Berbeco R and Makrigiorgos GM. 2014. Targeted radiotherapy with gold nanoparticles: current status and future perspectives. Nanomedicine $\mathbf{9 ( 7 )}$ 1063-82

[21] Yook S, Cai Z, Lu Y, Winnik MA, Pignol JP and Reilly RM 2016 Intratumorally Injected 177LuLabeled Gold Nanoparticles: Gold Nanoseed Brachytherapy with Application for Neoadjuvant Treatment of Locally Advanced Breast Cancer J. Nucl. Med. 57(6) 936-42

[22] Shi M, Paquette B, Thippayamontri T, Gendron L, Guérin B and Sanche L 2016 Increased radiosensitivity of colorectal tumors with intra-tumoralinjection of low dose of gold nanoparticles Int. J. Nanomed. 11 5323-5333 


\title{
Modeling of MNP Effects with Monte Carlo
}

\author{
Wonmo Sung ${ }^{1}$ and Benedikt Rudek ${ }^{1,2}$ \\ ${ }^{1}$ Massachusetts General Hospital \& Harvard Medical School, Boston, MA, USA \\ 2 Boston University, Boston, MA, USA
}

\section{Status}

The Monte Carlo (MC) method is a class of computational methods that simulates a process by randomly sampling its variables. For the modeling of Metallic Nanoparticle (MNP) effects, the radiation

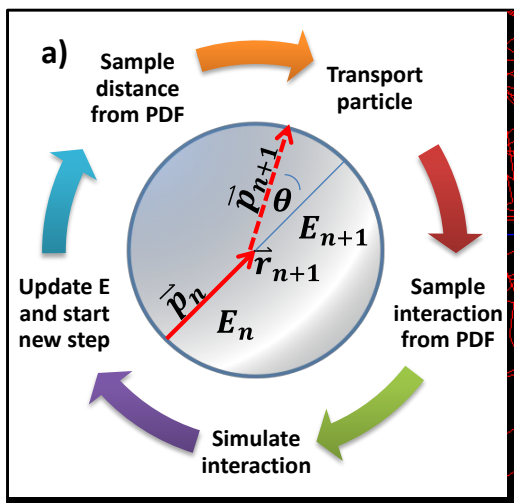

c)

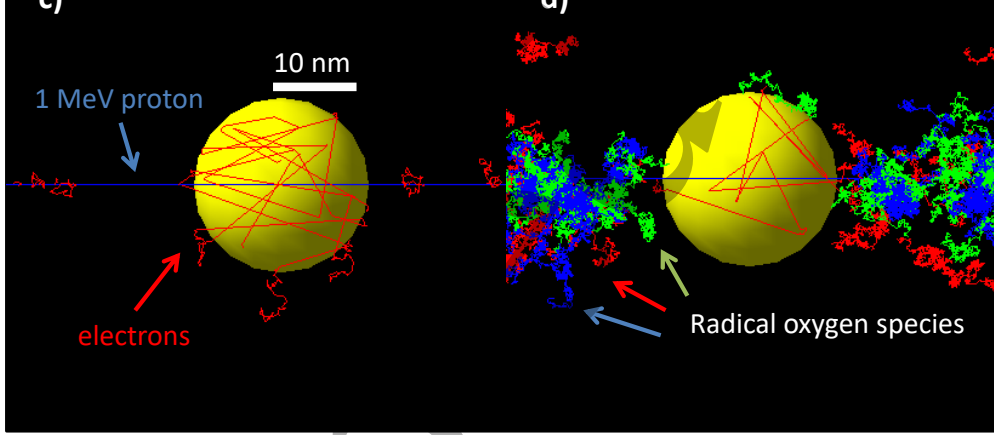

Fig. 1 a) MC codes simulate radiation transport by numerically calculating the change in position $\mathbf{r}$, momentum $\mathbf{p}$ and energy $\mathbf{E}$. The transport of a $1 \mathrm{MeV}$ proton through a $20 \mathrm{~nm}$ gold nanoparticle surrounded by water is shown for MC simulation using b) only $\mathrm{CH}$ physics processes in $10 \mathrm{~nm}$ steps (Geant4 Livermore EM physics), c) using TS in water (Geant4-DNA) and d) with water radiolysis included.

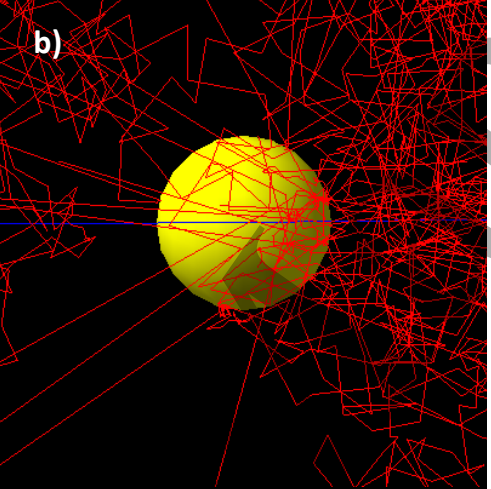

transport is simulated by sampling the probability density functions (PDFs) of various electromagnetic interactions. MC codes use two types of transport calculations: The condensed history $(\mathrm{CH})$ approach groups multiple scattering processes together to reduce computation time for larger volumes, whereas the discrete model simulates the track structure (TS) event-byevent to calculate radiation interactions stepwise on a microand nanometer-scale. Examples of TS codes are NOREC, PARTRAC, KURBUC, TRAX, NASIC, generalpurpose $\mathrm{MC}$ codes using $\mathrm{CH}$ are EGS and FLUKA, and PENELOPE, MCNP and Geant4, which have also been extended with TS simulation capabilities for to low-energy processes in cellular media (Incerti, 2016a and references therein). The Geant4-DNA TS extension, for example, simulates electron interactions down to a few electronvolt (eV) in liquid water and has 
become popular in the medical physics research community as its features are also readily available through user-friendly interfaces such as TOPAS and GATE. MC simulations have been used to obtain electron emission spectra and radial dose distributions for single or clustered MNPs $\left({ }_{26} \mathrm{Fe},{ }_{47} \mathrm{Ag},{ }_{64} \mathrm{Gd}\right.$, ${ }_{78} \mathrm{Pt}$ and ${ }_{79} \mathrm{Au}$ ) for various geometrical parameters and radiation modalities (Zygmanski and Sajo, 2016, McMahon et al 2015).

While the physical dose enhancement by MNPs might be negligible for megavolt photons and particle beams, their impact in the chemical stage might be significant (Rudek et al 2019). Several MC codes offer simulation of radiolysis in a pure water environment (PARTRAC, Geant4-DNA, TRAXchem, KURBUCchem, RITRACKS and TOPAS-nBio). The increase in yield of radical oxygen species(ROS) scales with the physical dose enhancement when radial dose distributions are folded with radiolysis simulations in the surrounding water (Tran et al 2016). Experimental work demonstrated adverse effects such as catalytical production of hydroxyl at GNPs and binding of scavengers but also beneficial effects such as a reduction of ROS by superoxide dismutase or peroxide catalase. The actual chemical reactions of ROS and scavengers at the MNP surface are so far ignored in all MC codes. As MC codes already struggle with computational costs of pure water radiolysis, an implementation of such surface reactions that depend on dose, temperature and the chemical environment will dramatically increase the parameter phase space and is unlikely to happen anytime soon.

Recently, the microscopic dose distribution shifted into focus to investigate biological effects of GNP distributions on cellular structures. The sensitivity enhancement predicted with an adaptation of the local effect model produced higher sensitization with kilovolt photons if GNPs were internalized into the cell (McMahon et al 2011). However, sensitization for megavolt photons and protons was only significant when GNPs were internalized into the nucleus (Lin et al 2015a). Organelles other than the nucleus such as the mitochondrial DNA may also be considered a target because some GNP configurations accumulate on the surface of mitochondria leading to locally increased energy deposition within the organelle (McNamara et al 2016, Kirkby and Ghasroddashti 2015). Imaging the experimental GNP distributions as input for MC simulations showed how important the geometry of radiosensitive structures and GNP clustering is for the prediction of cell survival (Sung et al 2018). Similarly, high dose spikes at the vascular wall, especially for hypo-fractionated treatment, have been found for accumulation of GNPS in the vesicles as typically observed in in-vivo experiments (Lin et al 2015b).

Simulations of macroscopic GNP distributions have also been carried out to show pathways of clinical translation. In virtual phantom scenarios, photon beam energies were optimized for arc therapy, and found to be dependent on GNP uptake, beam arrangements, and patient geometries (Koger and Kirkby 2018, Sung and Schuemann 2018). 


\section{Current and future challenges}

Currently, TS MC codes are limited to simulations in water. Physics models need to consider the full deexcitation of electronically-excited heavy elements such as via long Auger cascades, simulate discrete ionization events and electron transport with low cut-off energies and benchmark these processes with updated cross section databases and scattering experiments on metallic nanostructures.

In addition, more systematic experiments are necessary to describe the chemical processes with MNPs. $M C$ codes need to be extended to the chemical stage as recent experiments point out a significant impact of nanoparticles on radiolysis and scavenging of ROS. The implementation of selected test cases orientated on experimental benchmarks promises a major step forward in understanding the indirect effect by MNPs.

One significant challenge is that the calculation speed is slow not only for chemistry but already when only taking into account discrete physical processes compared to the condensed history approach.

Ultimately, the $\mathrm{MC}$ codes will be compared to experiments in living material. Cellular structural information is still limited due to current imaging capabilities. For example, transmission electron microscopy (TEM) only provides 2D structures for dead cells. Fluorescence probes may alter the original physiological conditions of the cell. Moreover, those studies are restricted only for in-vitro cell experiments.

\section{Advances in science and technology to meet challenges}

Recently, the full deexcitation cascade in GNPs can be activated in Geant4, however, the initial ionization is limited to the $\mathrm{K}, \mathrm{L}$ and $\mathrm{M}$ shells and the increase in total emitted energy was below $1 \%$ for all tested modalities (Incerti, 2016b). Discrete electron transport in gold has been implemented in Geant4-DNA and will be available in a future release (Sakata et al 2018). Recent versions of MCNP6 and PENELOPE include extended cross-sections down to $10 \mathrm{eV}$ and $50 \mathrm{eV}$ and calculate slightly higher doses than Geant4-DNA within the first few $\mathrm{nm}$ from the surface of the GNP (Jung et al 2018). Discrete electron transport in copper is available in PARTRAC (Dingfelder and Travia 2015) and an extension to gold is expected soon. However, molecular dynamics approaches such as the MBN Explorer will possibly allow to analyze the structure, composition and dynamics of atomic clusters and coated nanoparticles (Solov'yov et al 2012).

For ionization by particle beams, a discretization is not yet implemented in Geant 4 and the cut-off at rather high mean ionization energies in the Bethe-Bloch theory for heavy elements compared to light elements is a possible source for the underestimation of dose enhancement observed for MNPs. A 
discretized ionization model based on cross sections calculated in the Binary Encounter Approximation as used in TRAX showed an increase of low-energy electron emission $(<1 \mathrm{keV})$ of up to two orders of magnitude (Hespeels, 2019) which will push the overall dose enhancement closer to enhancements observed in cell experiments with particle beams. An inter-comparison such as the recent exercise by the EUropean RAdiation DOSimetry (EURADOS) group is a helpful approach to ensure quality control of new implementations and various available codes. (Li et al n.d.)

Additionally, full track structure simulations with a mechanistic approach will offer a different method to the phenomenological models in the simulation setup. This approach may provide more mechanistic biological endpoints such as DNA breakages (J Schuemann et al 2018).

To improve the computational speed, new codes written for Graphics Processing Unit (GPU) cards have been developed and cloud computing, wherein computing resources are allocated from a third party, can also resolve the computational costs in track structure Monte Carlo simulations (Wang et al n.d.). For a more realistic simulation setup, advanced molecular imaging is necessary to acquire more accurate 4D cellular structures. Clinical trials of nanoparticle-mediated radiotherapy will offer data on tumor control probability and normal tissue toxicity. Using imaged biodistributions in Monte Carlo simulations will help to interpret these new data.

\section{Concluding remarks}

Monte Carlo simulations provide a comprehensive description of physical dose enhancements by MNPs. Advances in the physics modeling will further improve the accuracy especially in the low-energy range. The future challenge, however, lies in the implementation of indirect pathways via ROS and the modeling of biological damage and repair. Both approaches require substantial improvement of the underlying models, the available cross sections and the efficiency of the calculation. Future Monte Carlo studies should then be carefully designed to complement in-vivo and in-vitro observations.

\section{Acknowledgments}

BR acknowledges support from the German Research Foundation (DFG), project no. RU 2197/1-1.

\section{References}

1. Dingfelder M, and Travia A (2015). Cross sections for track structure codes: Volume versus surface transport. Radiation Protection Dosimetry, 166(1-4), 10-14.

2. Hespeels F, Lucas S, Tabarrant T, Scifoni E, Kraemer M, Chêne G., Strivay D, Tran H N, and Heuskin A-C (2019). Experimental measurements validate the use of the binary encounter 
approximation model to accurately compute proton induced dose and radiolysis enhancement from gold nanoparticles. Physics in Medicine and Biology, 64(6), 065014.

3. Incerti S, Douglass M, Penfold S, Guatelli S, and Bezak E (2016a). Review of Geant4-DNA applications for micro and nanoscale simulations. Physica Medica, 32 (10) 1187-1200.

4. Incerti S, Suerfu B, Xu J, Ivantchenko V, Mantero A, Brown J M C, Bernal M A, Francis Z, Karamitros M,Tran H N (2016b). Simulation of Auger electron emission from nanometer-size gold targets using the Geant4 Monte Carlo simulation toolkit. Nuclear Instruments and Methods in Physics Research, 372, 91-101.

5. Jung S, Sung W and Ye S-J 2018 Low-energy electron dose-point kernels and radial dose distributions around gold nanoparticles: Comparison between MCNP6.1,PENELOPE2014 and Geant4-DNA Nuclear Inst. and Methods in Physics Research, B 43018-22

6. Kirkby C and Ghasroddashti E 2015 Targeting mitochondria in cancer cells using gold nanoparticle-enhanced radiotherapy: A Monte Carlo study Medical Physics, 42(2), 11191128

7. Koger B and Kirkby C 2018 Optimization of photon beam energies in gold nanoparticle enhanced arc radiation therapy using Monte Carlo methods Physics in Medicine \& Biology 8839-53

8. Li W B, Belchior A, Beuve M, Chen Y Z, Di Maria S, Friedland W, Gervais B, Heide B, Hocine N, Ipatov A, Klapproth A P, Li CY, Li J L, Multhoff G, Poignant F, Rabus H, Rudek B, Schuemann J, Testa $\mathrm{E}$, Villagrasa $\mathrm{C}$ and Xie W Z Intercomparison of simulated physical radiation quantities for gold nanoparticles irradiated by $\mathrm{x}$-rays with multiple Monte Carlo simulation codes for dosimetric quality assurance submitted

9. Lin Y, McMahon S J, Paganetti H and Schuemann J 2015a Biological modeling of gold nanoparticle enhanced radiotherapy for proton therapy Physics in Medicine \& Biology 60 4149-68

10. Lin Y, Paganetti H, McMahon S J and Schuemann J 2015b Gold nanoparticle induced vasculature damage in radiotherapy: Comparing protons, megavoltage photons, and kilovoltage photons Med. Phys. 42 5890-902 
11. McMahon S J, Hyland WB, Muir M F, Coulter, J A, Jain S, Butterworth K T, Schettino G, Dickson G R, Hounsell A R, O'Sullivan J M, Prise K M, Hirst D G and Currell F J 2011 Biological consequences of nanoscale energy deposition near irradiated heavy atom nanoparticles. Scientific Reports 118

12. McMahon SJ, Paganetti H and Prise KM 2015 Optimising element choice for nanoparticle radiosensitisers Nanoscale, 8, 581-589

13. McNamara A L, Kam W W Y, Scales N, McMahon S J, Bennett J W, Byrne H L, Schuemann J, Paganetti H, Banati R and Kuncic Z 2016 Dose enhancement effects to the nucleus and mitochondria from gold nanoparticles in the cytosol Physics in Medicine \& Biology 59936010

14. Rudek B, McNamara A, Ramos-Mendez J, Byrne H L, Kuncic Z and Schuemann J 2019 Radioenhancement by gold nanoparticles and their impact on water radiolysis for $\mathrm{x}$-ray, proton and carbon-ion beams Physics in Medicine and Biologyaccepted manuscript

15. Sakata D, Kyriakou I, Okada S, Tran, Hoang N., Lampe N, Guatelli S, Bordage M C, Ivanchenko V, Murakami K, Sasaki T, Emfietzoglou D and Incerti S 2018 Geant4-DNA track-structure simulations for gold nanoparticles: The importance of electron discrete models in nanometer volumes. Medical Physics 45(5) 2230-2242

16. Schuemann J, McNamara A L, Ramos-Méndez J, Perl J, Held K D, Paganetti H, Incerti S and Faddegon B 2018 TOPAS-nBio: An Extension to the TOPAS Simulation Toolkit for Cellular and Sub-cellular Radiobiology Radiation Research 191125

17. Solov'yov I A, Yakubovich A V, Nikolaev P V, Volkovets I and Solov'yov A V 2012 MesoBioNano explorer-A universal program for multiscale computer simulations of complex molecular structure and dynamics Journal of Computational Chemistry 33 2412-39

18. Sung $\mathrm{W}$ and Schuemann J 2018 Energy optimization in gold nanoparticle enhanced radiation therapy Physics in Medicine \& Biology 63135001

19. Sung W, Jeong Y, Kim H, Jeong H, Grassberger C, Jung S, Ahn G-O, Han Kim II, Schuemann J, Lee K and Ye S-J 2018 Computational Modeling and Clonogenic Assay for Radioenhancement of Gold Nanoparticles Using 3D Live Cell Images Radiation Research 190 558-64 
20. Tran H N, Karamitros M, Ivanchenko V N, Guatelli S, McKinnon S, Murakami K, Murakami K, Sasaki T, Okada S, Bordage M, C, Francis Z, El Bitar Z, Bernal M A, Shin J I, Lee S B, Barberet Ph, Tran T T, Brown J M C, Nhan Hao T V, and Incerti, S 2016 Geant4 Monte Carlo simulation of absorbed dose and radiolysis yields enhancement from a gold nanoparticle under MeV proton irradiation Nuclear Instruments and Methods in Physics Research 373, 126-139.

21. Wang H, Ma Y, Pratx G, Xing L 2011 Toward real-time Monte Carlo simulation using a commercial cloud computing infrastructure Physics in Medicine and Biology N175-81

22. Zygmanski P and Sajo E 2016 Nanoscale radiation transport and clinical beam modeling for gold nanoparticle dose enhanced radiotherapy (GNPT) using X-rays The British Journal of Radiology, 89(1059), 20150200. 


\title{
Chemical Effects in NPs Induced Radio-
}

\section{Enhancement}

Sandrine Lacombe, ${ }^{1}$ Vincent Favaudon, ${ }^{2}$ Erika Porcel, ${ }^{1}$

${ }^{1}$ ISMO (UMR 8625), University Paris-Saclay, University Paris Sud, CNRS, 91405 Orsay Cedex, France

${ }^{2}$ Institut Curie, Inserm U 1020-CNRS UMR 3347, University Paris-Saclay, University Paris Sud, 91405

Orsay Cedex, France

\begin{abstract}
:
Nanoparticle enhanced radiation therapy is a breakthrough in the development of cancer treatment, which mainly aims at enhancing radiation effects (called radio-enhancement) in tumors selectively and can at the same time be used for tumor diagnosis or drug delivery using the same agent. The underlying principle of NP radio-enhancement is the amplification of radiation induced electron emission into a nanoscale volume using efficient electron emitters such as high-Z compounds, thereby amplifying the induction of lethal (complex) biological damage and the biological efficiency of radiation treatments. Various experimental and theoretical studies consolidated this description. However, the role of water radicals issued from the interaction of electron with surrounding water molecules, and, more surprisingly, the influence of molecular oxygen on the chemical reactions consecutive to the NP activation, are not yet much described in the existing models. This overview summarizes the chemistry that counts for the radio-enhancing effects of NPs.
\end{abstract}

Status:

Tumor targeting of radiotherapy treatments and improvement of the therapeutic index for radioresistant cases remain great challenges. Adding nanoparticles able to improve radiation effects (radio-enhancers) into tumors is proposed as a promising strategy. The principle of this method is to use high electron emitters to amplify the local electron emission when activated by ionizing radiation. So far, complexes or nanoagents composed of high-Z atoms such as gold, platinum, gadolinium (AGuIX in particular, see Lux et al. (Lux et al 2018)) or hafnium have proven to be efficient radio-enhancers (Lux et al 2018, Porcel et al 2010, Bonvalot et al 2017). The advantage of small nanoparticles compared to metallic complexes stems from their capacity to concentrate in tumors via the Enhanced and Permeability Retention Effect (EPR). They also offer a high flexibility of surface functionalization, which allows implementing various modalities within the same agent (MRI by adding paramagnetic atoms, 
PET using isotopes, cell imaging or other fluorescent based techniques by grafting various fluorescent markers) (Truillet et al 2016). The fast development of a radio-enhancement strategy - already used in clinic (see F. Lux et al. (Lux et al 2018)) - requires better understanding and simulation of the phenomenon with the goal to improve the design of new nanoagents and an implementation of this strategy in treatment planning systems. The difficulty of this work stems from the multiscale character of the phenomenon ranging from the early stage activation of the processes $\left(<10^{-16} \mathrm{sec}\right.$, nanoscale) to the impact in vivo (minutes to hours, macroscale). In between these scales sits the chemistry stage, which is often absent from the models although it is expected to play a crucial role in the observed outcome.

Below, the stages of radio-enhancement in the presence of NPs are described. The third and fourth steps are present in all radiation schemes of biological systems but were not yet investigated in the presence of NPs. Figure 1 illustrates the physical and chemical stages expected in the NPs induced radio-enhancement.

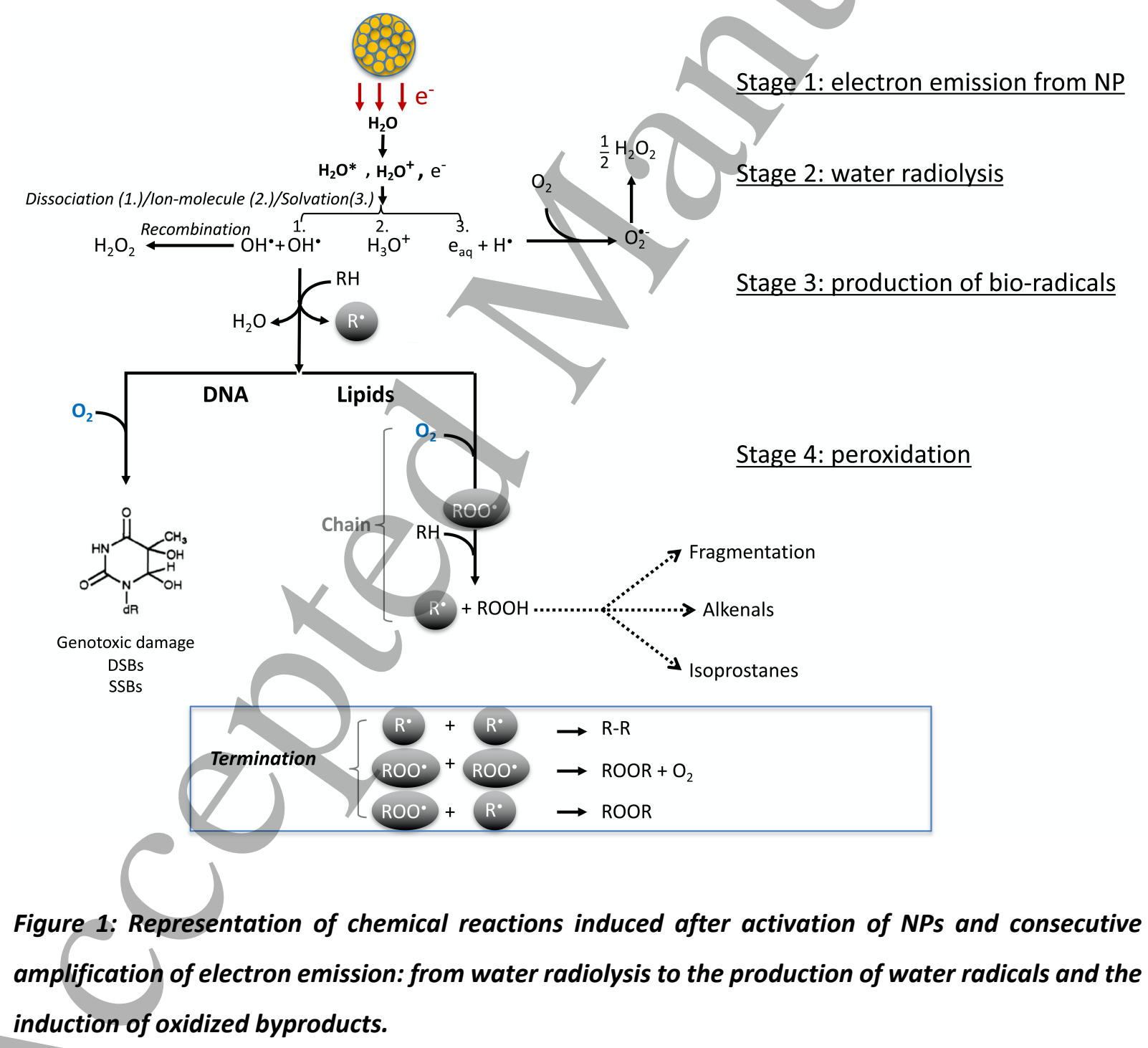

\section{induction of oxidized byproducts.}




\section{Step 1: Physical stage}

The physical stage of the radio-enhancement effect has been extensively described and reviewed (Kuncic and Lacombe 2018).

Briefly, the radio-enhancement is attributed to a local amplification of electron emission due to the high ionization cross section of high-Z atoms compared to water. The activation of NPs by incident photons is initiated via the photoelectric effect for photons of energies below approx $100 \mathrm{keV}$, and Compton effects for photons of higher energies. NPs may also be activated by incident charged particles, namely fast ions (protons or carbon ions as used in proton-and hadron-therapy), or electrons produced in the medium. In this case, Coulombic interactions are responsible for the activation of NPs (Kuncic and Lacombe 2018, Lacombe et al 2017). This model explains the improvement of radiation effects observed when NPs are added in the treatment of animals by x-rays or protons (Hainfeld et al 2004, Kim et al 2010). This effect was found to be correlated with the amplification of tumor cell killing when cells are treated with NPs prior to irradiation (Butterworth et al 2010). Using biomolecular probes in solution (without any metabolic effect), it was shown that the induction of nanosize lesions is enhanced in the presence of NPs (Butterworth et al 2008, Porcel et a/ 2012). This demonstrated the physical character of the NPs induced amplification of radiation lethality.

\section{Step 2: Chemical stage - water radiolysis}

The production of water radicals by photon, ion or electron irradiations and the impact on living organisms is a very well-known phenomenon. In contrast, very few studies focused on the relative role of water radicals versus the role of the direct interaction with incident radiation in the radioenhancement effect. The amplification of the radical production by NPs has been evidenced in experiments performed in water (Sicard-Roselli et al 2014) and in solution containing biomolecules (Porcel et al 2010). The few studies performed with cells using radical scavengers reported that, on average, hydroxyl radicals $(\mathrm{OH})$, the most powerful oxidants of water derivatives $\left(\mathrm{E}^{\circ}=\left({ }^{\circ} \mathrm{OH} / \mathrm{OH}^{-}\right)=1.90\right.$ $\mathrm{V}$ at $\mathrm{pH}=7$ ), account for $60-90 \%$ of the amplification of cell killing induced by high- $\mathrm{Z}$ compounds activated by high energy photons or ions (Usami et al 2008, Jeynes et al 2014). In parallel, it was demonstrated that the increase of nanosize lesions (>2 nm) is also due to $\mathrm{OH}$ (Porcel et al 2010, Butterworth et al 2008, Porcel et al 2012). So, the production of hydroxyl radicals is a key component of the radio-enhancement phenomenon. So far, very few simulation studies exist that consider this stage in simulation models (e.g. Geant 4 DNA (Schuemann et al 2019) and references therein, and TRAX for incident ion effects (Boscolo et al 2018)).

\section{Step 3 and 4: Chemical stage - production of organic radicals and peroxidation}


The chemistry that follows the production of hydroxyl radicals, strongly depends on the presence of molecular oxygen in biological systems. This phenomenon is well documented (Chapman et al 1973, Edwards et al 1984). However, the influence of NPs on these chemical reactions has never been studied nor even considered as important factor. In the view of future prospective, we remind here the basic principles of the chemistry that could be impacted by the presence of NPs.

Hydroxyl radicals $\left({ }^{\circ} \mathrm{OH}\right)$ produced during the radiolysis phase (step 2) rapidly and efficiently undergo reactions with the main organic constituents $(\mathrm{RH})$ of biological systems, namely, nucleic acids, proteins and lipids. These reactions lead to the production of carbon-centred radicals $\left(R^{\circ}\right)$ through hydrogen atom abstraction of a range of aliphatic or conjugated substrates $R H$. These species $\left(R^{*}\right)$ are highly reactive and short-lived ( $t_{1 / 2}<5$ ms in mammalian cells in complete absence of oxygen). $R^{\bullet}$ have a high affinity for $\mathrm{O}_{2}$ yielding peroxyradicals ( $\mathrm{ROO}^{\circ}$ ). This reaction is in competition with the annihilation of $\mathrm{R}^{*}$ by electron donors (radical scavengers) that afford a high degree of radioprotection.

The interaction of hydroxyl radicals ('OH) with DNA (Chapman et al 1973, Cadet et al 2005) and with lipids (Sevanian and Hochstein 1985) has been extensively reported. Peroxyradicals formed from DNA (ROO` with R corresponding to a nucleic acid chain) decay through bimolecular, tetroxide-based pathways in pyrimidines or 2'-deoxyribose ending in alcohols, aldehydes, cetones or bond breakage with frequent release of superoxide $\left(\mathrm{O}_{2}{ }^{\circ-}\right)$ and oxygen in the singlet excited state $\left({ }^{1} \mathrm{O}_{2}\right)$. Owing to lateral diffusion in phospholipid membranes, peroxyradicals formed from unsaturated lipids, namely lipid peroxyl radicals ( $\mathrm{ROO}^{\circ}$ with $\mathrm{R}$ corresponding to a lipidic chain) are able to abstract a hydrogen atom from other lipid molecules initiating a chain reaction. Chain termination is achieved by interaction of $\mathrm{ROO}^{\circ}$ with scavengers (i.e. $\mathrm{H}^{*}$ ) yielding alkyl hydroperoxides $(\mathrm{ROOH})$ or by radical-radical recombination yielding organic peroxides (ROOR) (Sonntag 1987). Disproportionation may also take place. Little is known of the fate of peroxyradicals formed from proteins. The well-known radiosensitizing effect of oxygen stems from these chemical cascades. Consistently, the partial pressure of oxygen in irradiated tissues is a crucial determinant of radiosensitivity. $\mathrm{pO}_{2}$ ranges between 1 and $10 \%$ in normal tissues, which defines normoxia, whilst the presence of hypoxic regions in solid tumors $\left(\mathrm{pO}_{2}<0.5 \%\right)$ explains the low therapeutic index of radiotherapy.

One study already reported that NPs have low effect when cells are irradiated in anoxic conditions $\left(\mathrm{pO}_{2}\right.$ close to 0) (Jain et al 2014). This result questions the efficiency of NPs to improve the treatment of hypoxic cancer cases. Thus, further experiments are needed to better characterize and quantify the effects of NPs depending on the cell type (if any) and their chemical environment. The localization of NPs in cell compartments (nucleus, lysosomes, mitochondria) may influence this effect. 
In summary, the chemistry involved in NP induced radio-enhancement is crucial to characterize with the goal of designing more competitive agents and monitoring their impact in various tissues. This requires new investigations and theoretical models, which enclose reactions driven by water radicals and by molecular oxygen.

\section{Acknowledgements}

This work was supported by the European Commission with the project ARGENT (FP7PEOPLE-2013-ITN-608163), by the Université Paris Saclay with the "Initiative de Recherche Stratégique" (IRS) NanoTheRad.

\section{References}

Bonvalot S, Le Pechoux C, De Baere T, Kantor G, Buy X, Stoeckle E, Terrier P, Sargos P, Coindre J M, Lassau N, Ait Sarkouh R, Dimitriu M, Borghi E, Levy L, Deutsch E and Soria J-C 2017 First-inHuman Study Testing a New Radioenhancer Using Nanoparticles (NBTXR3) Activated by Radiation Therapy in Patients with Locally Advanced Soft Tissue Sarcomas. Clinical Cancer Research 23 908-17

Boscolo D, Krämer M, Durante M, Fuss M C and Scifoni E 2018 TRAX-CHEM: A pre-chemical and chemical stage extension of the particle track structure code TRAX in water targets Chem. Phys. Lett. $69811-8$

Butterworth K T, Coulter J A, Jain S, Forker J, McMahon S J, Schettino G, Prise K M, Currell F J and Hirst D G 2010 Evaluation of cytotoxicity and radiation enhancement using $1.9 \mathrm{~nm}$ gold particles: potential application for cancer therapy. Nanotechnology 21295101

Butterworth K T, Wyer J A, Brennan-Fournet M, Latimer C J, Shah M B, Currell F J and Hirst D G 2008 Variation of strand break yield for plasmid DNA irradiated with high-Z metal nanoparticles. Radiat. Res. $170381-7$

Cadet J, Douki T, Gasparutto D and Ravanat J-L 2005 Radiation-induced damage to cellular DNA: measurement and biological role Radiat. Phys. Chem. 72 293-9

Chapman J D, Reuvers A P, Borsa J and Greenstock C L 1973 Chemical radioprotection and radiosensitization of mammalian cells growing in vitro. Radiat. Res. 56 291-306 
Edwards J C, Chapman D, Cramp W A and Yatvin M B 1984 The effects of ionizing radiation on biomembrane structure and function. Prog. Biophys. Mol. Biol. 43 71-93

Hainfeld J F, Slatkin D N and Smilowitz H M 2004 The use of gold nanoparticles to enhance radiotherapy in mice. Phys. Med. Biol. 49 N309-15

Jain S, Coulter J A, Butterworth K T, Hounsell A R, McMahon S J, Hyland W B, Muir M F, Dickson G R, Prise K M, Currell F J, Hirst D G and O'Sullivan J M 2014 Gold nanoparticle cellular uptake, toxicity and radiosensitisation in hypoxic conditions. Radiother Oncol $110342-7$

Jeynes J C G, Merchant M J, Spindler A, Wera A-C and Kirkby K J 2014 Investigation of gold nanoparticle radiosensitization mechanisms using a free radical scavenger and protons of different energies. Phys. Med. Biol. 59 6431-43

Kim J-K, Seo S-J, Kim K-H, Kim T-J, Chung M-H, Kim K-R and Yang T-K 2010 Therapeutic application of metallic nanoparticles combined with particle-induced $\mathrm{x}$-ray emission effect Nanotechnology $\mathbf{2 1}$ 425102

Kuncic Z and Lacombe S 2018 Nanoparticle radio-enhancement: principles, progress and application to cancer treatment Phys. Med. Biol. 63 02TR01

Lacombe S, Porcel E and Scifoni E 2017 Particle therapy and nanomedicine: state of art and research perspectives. Cancer Nano 89

Lux F, Tran V L, Thomas E, Dufort S, Rossetti F, Martini M, Truillet C, Doussineau T, Bort G, Denat F, Boschetti F, Angelovski G, Detappe A, Crémillieux Y, Mignet N, Doan B-T, Larrat B, Meriaux S, Barbier E, Roux S, Fries P, Müller A, Abadjian M-C, Anderson C, Canet-Soulas E, Bouziotis P, Barberi-Heyob M, Frochot C, Verry C, Balosso J, Evans M, Sidi-Boumedine J, Janier M, Butterworth K, McMahon S, Prise K, Aloy M-T, Ardail D, Rodriguez-Lafrasse C, Porcel E, Lacombe S, Berbeco R, Allouch A, Perfettini J-L, Chargari C, Deutsch E, Le Duc G and Tillement O 2018 AGuIX ${ }^{\circledast}$ from bench to bedside-Transfer of an ultrasmall theranostic gadolinium-based nanoparticle to clinical medícine. Br J Radiol 20180365

Porcel E, Li S, Usami N, Remita H, Furusawa Y, Kobayashi K, SECH C L and Lacombe S 2012 NanoSensitization under gamma rays and fast ion radiation J. Phys.: Conf. Ser. 373012006 
Porcel E, Liehn S, Remita H, Usami N, Kobayashi K, Furusawa Y, Sech C L and Lacombe S 2010 Platinum nanoparticles: a promising material for future cancer therapy? Nanotechnology 21 085103

Schuemann J, McNamara A L, Warmenhoven J W, Henthorn N T, Kirkby K J, Merchant M J, Ingram S, Paganetti H, Held K D, Ramos-Méndez J, Faddegon B, Perl J, Goodhead D T, Plante I, Rabus H, Nettelbeck H, Friedland W, Kundrat P, Ottolenghi A, Baiocco G, Barbieri S, Dingfelder M, Incerti S, Villagrasa C, Bueno M, Bernal M A, Guatelli S, Sakata D, Brown J M C, Francis Z, Kyriakou I, Lampe N, Ballarini F, Carante M P, Davidkova M, Stepan V, Jia X, Cucinotta FA, Schulte R, Stewart R D, Carlson D J, Galer S, Kuncic Z, Lacombe S, Milligan J, Cho S H, Sawakuchi G, Inaniwa T, Sato T, Li W, Solov'yov A V, Surdutovich E, Durante M, Prise K M and McMahon S J 2019 A New Standard DNA Damage (SDD) Data Format Radiat. Res. 19176

Sevanian A and Hochstein P 1985 Mechanisms and consequences of lipid peroxidation in biological systems. Annu. Rev. Nutr. 5 365-90

Sicard-Roselli C, Brun E, Gilles M, Baldacchino G, Kelsey C, McQuaid H, Polin C, Wardlow N and Currell F 2014 A new mechanism for hydroxyl radicál production in irradiated nanoparticle solutions. Small $103338-46$

Sonntag C 1987 The Chemical Basis of Radiation Biology (Taylor and Francis: London)

Truillet C, Thomas E, Lux F, Huynh LT, Tillement O and Evans M J 2016 Synthesis and Characterization of (89)Zr-Labeled Ultrasmall Nanoparticles. Mol. Pharmaceutics 13 2596-601

Usami N, Furusawa Y, Kobayashi K, Lacombe S, Reynaud-Angelin A, Sage E, Wu T-D, Croisy A, Guerquin-Kern J-L and Le Sech C 2008 Mammalian cells loaded with platinum-containing molecules are sensitized to fast atomic ions. Int J Radiat Bio/ 84 603-11 


\title{
Modelling the Biological Effects of Metal
}

\section{Nanoparticles}

\author{
Stephen McMahon ${ }^{1}$ \\ ${ }^{1}$ Queen's University Belfast, 97 Lisburn Road, Belfast, BT9 7AE
}

\section{Status}

The use of metal nanoparticles to enhance radiation therapy was originally proposed on very simple physical grounds - due to their high density and atomic number, heavy metals absorbed significantly more radiation than soft tissue, increasing the dose deposited and improving clinical outcomes. This effect can be readily quantified using Monte Carlo modelling on the macro- (Cho 2005, McMahon et al 2008) and nano-scales (Jones et al 2010, McMahon et al 2011).

However, while significant radiosensitisation was observed experimentally, it rapidly became clear that the observed effects differed significantly from those predicted on this simple physical basis. Two major observations were made: i) many experiments reported significantly greater enhancements than those predicted by physical dose modification alone; and ii) significant enhancement was observed with megavoltage photon irradiations, with only negligible differences in dose deposition (Chithrani et al 2010, Jain et al 2010, Butterworth et al 2012). These observations have been confirmed in numerous systems, clearly demonstrating that biological processes underlie experimental radiosensitisation, schematically illustrated in Figure 1. However, the exact nature of these processes is not well known. A range of different possibilities have been implicated by different studies, with some of the main candidates illustrated in Figure 1.

Additional sensitisation due to nanoscale dose enhancement has been by far the most extensively modelled process. As described elsewhere, models of MNP-radiation interactions make it clear that they have a highly heterogeneous impact on dose distributions on the nanoscale (Jones et al 2010, McMahon et al 2011). Analogies were drawn between these dose distributions and charged particle therapy, where similar sub-cellular localisation of dose leads to greater biological effects (Christian et al 2018). 
These effects have been extensively studied in charged particle therapy, with a number of models proposed to understand these effects, such as the Local Effect Model (LEM) (Elsässer et al 2008) and the Microdosimetric Kinetic Model (MKM) (Hawkins 2003). These models have been applied to GNP-enhanced therapy, and demonstrated MNP dose heterogeneity leads to greater biological effects and increased sensitisation for a range of radiation types (McMahon et al 2011, Lechtman et al 2013, Lin et al 2014).

However, such approaches involve assumptions about the uptake and distribution of MNPs throughout the cell which are not readily verified experimentally. While some progress has been made through the use of detailed cell-level imaging enabling the prediction of cellspecific dose distributions and associated radio-sensitisation effects, it is increasingly apparent that nanoscale dosimetry cannot explain the full variation of experimental data.

A major limitation of these models is that they typically consider MNPs as otherwise inert modifiers of radiation dose, in contrast with experimental data showing that MNPs can modify a range of chemical and biological processes. These effects may further modulate the radiosensitivity of cells. For example, many nanoparticle preparations have significant physico-chemical activity, acting as both sources and scavengers of free radicals (Ionita et al 2008), which is known to significantly impact on the yield and type of DNA damage, potentially driving sensitisation at all energies.

Even in the absence of radiation, MNPs are not biologically inert, and have been shown to impact on cells in a range of lethal and sub-lethal fashions. These include the induction of DNA damage as single agents (Kang et al 2010), upregulation of cell death pathways (Djurišić et al 2015), and disruption of oxidative stress regulation (Dayem et al 2017). These effects have been shown to sensitise cells to other DNA damaging agents, underscoring that MNPs can affect response even in the absence of physical dose modification (Zheng and Sanche 2009, Jain et al 2010). 


\section{Current and Future Challenges}

Figure 1 Left: Interplay between physical and biological sensitisation. Although MeV physical enhancement is expected to be only a few percent of keV enhancement, megavoltage sensitisation has been reported (squares: Chithrani 2010, circles: Jain 2010), indicating a large component of biological sensitisation. The absolute and relative magnitudes of the physical and biological effects are strongly MNP dependent, and are not easy to extrapolate between systems. Right: Illustration of possible drivers of radiationsensitisation in MNP-treated cells. These range from well-understood physical processes (top) to biological effects with almost no modelling (bottom).

Despite the importance of these effects- particularly at clinically-relevant energies - they are much less understood than physical dosimetry, and have seen little or no quantitative modelling. This represents a significant limitation in our understanding of MNP-based therapies.

A major challenge in modelling MNP sensitisation is the extreme heterogeneity of particle types and model systems. While a small number/of commercial MNPs have been used in multiple studies, most studies use locally-produced nanoparticles, frequently with specifically developed coatings and targeting molecules. While the dosimetric impact of these particles is not significantly affected by this functionalisation, it can profoundly change their biological activity. While uptake and localisation can be incorporated in physical dosimetry, purely biological effects are not meaningfully represented. In addition, the majority of published studies focuses on limited endpoints (e.g. cell killing). Together, this means there is insufficient evidence to fully characterise cellular toxicity and radiation sensitisation, and identify underlying mechanisms for each nanoparticle preparation. This challenge is compounded by the difficulty of translating in vitro observations into in vivo systems. Here, differences in the pharmacokinetics of different preparations and their impact through the course of a full treatment schedule must also be taken into account.

As a result, modelling in this area remains under-conditioned, with ad-hoc analysis of limited datasets producing models which are in reasonably good agreement with fitting data, but 
which do not translate effectively to other systems. This approach is unlikely to generate models with wider applicability to support the development and optimisation of novel MNP treatments.

\section{Advances in science and technology to meet challenges}

The most efficient way to characterise a single nanoparticle preparation, remains an ad-hoc approach - that is, treating the nanoparticles as simple dose-modifying agents, using standard techniques to measure the additive and synergistic effects of the MNPs with radiation, and fitting a suitable empirical model. This has the advantage of being simple, and less experimentally demanding. Studies of the dependence of MNP radiosensitisation on irradiation type and energy may also enable a separation of "physical" and "biological" components of sensitisation. However, such ad-hoc approaches are not suitable for more general predictive tools. Instead, more general predictions require the development of relevant, systematic, mechanistic models to enable the integration of many heterogeneous datasets to produce useful predictions for a range of MNP.

A number of groups are beginning to develop tools which may address some of these limitations. For example, realistic Monte Carlo models of cellular DNA may enable the accurate prediction of radiation-induced DNA damage, integrated with models of free radical chemistry to incorporate radiation indirect effects (Meylan et al 2017, Lampe et al 2018). As these models mature, they may offer the capacity to incorporate MNPs as mediators of both direct and indirect damage providing a natural opportunity to understand these effects.

By contrast, models of purely biological mechanisms of MNP radiosensitisation remain limited. Although a number of individual models have considered some mechanistic aspects of DNA repair (Taleei and Nikjoo 2013, McMahon et al 2016, Henthorn et al 2017), in many cases these cannot easily incorporate the biological perturbations introduced by MNP. As a result, alternative approaches may be required to fully understand MNP radiosensitisation.

Systems biology and machine learning techniques present one alternative to more mechanistic model development. While these approaches have not seen wide application in MNP radiosensitisation, they have been applied to understand nanoparticle uptake and toxicity (Winkler et al 2014, Costa and Fadeel 2016). These may provide a framework to enable the complexity of cellular biology to be incorporated alongside mechanistic physical predictions in MNP radiosensitisation. 


\section{Concluding Remarks}

While it is clear that a large portion of MNP radiosensitisation is driven by biological effects which are largely independent of the physical dose modification, modelling in this area remains a significant weakness. Designing an optimised nanoparticle therapy necessitates the development of new approaches. This may involve the development of more mechanistic descriptions of MNP-cell-radiation interactions, or machine learning and systems biology approaches to leverage heterogeneous datasets for novel insights.

Regardless of the approach, there remains a pressing need for more high-quality, systematic experimental investigation of the impact of MNP across all stages of radiation response. Moreover, efforts should be made to present data in as accessible a format as possible, to enable incorporation of these disparate datasets in unified models.

\section{References}

Butterworth K T, McMahon S J, Currell F J and Prise K M 2012 Physical basis and biological mechanisms of gold nanoparticle radiosensitization. Nanoscale 4 4830-8 Online: http://www.ncbi.nlm.nih.gov/pubmed/22767423

Chithrani D B, Jelveh S, Jalali F, van Prooijen M, Allen C, Bristow R G, Hill R P and Jaffray D A 2010 Gold nanoparticles as radiation sensitizers in cancer therapy. Radiat. Res. 173 719-28 Online: http://www.ncbi.nlm.nih.gơv/pubmed/20518651

Cho S H 2005 Estimation of tumour dose enhancement due to gold nanoparticles during typical radiation treatments: a preliminary Monte Carlo study. Phys. Med. Biol. 50 N163-73 Online: http://www.ncbi.nlm.nih.gov/pubmed/16030374

Christian, Karger P and Peschke 2018 RBE and related modeling in carbon-ion therapy Phys. Med. Biol. 63 1-2 Online: http://iopscience.iop.org/article/10.1088/1361$6560 / \mathrm{aa} 9102 / \mathrm{pdf}$

Costa P M and Fadeel B 2016 Emerging systems biology approaches in nanotoxicology: Towards a mechanism-based understanding of nanomaterial hazard and risk Toxicol. Appl. Pharmacol. 299 101-11 Online: http://dx.doi.org/10.1016/j.taap.2015.12.014

Dayem A A, Hossain M K, Lee S Bin, Kim K, Saha S K, Yang G M, Choi H Y and Cho S G 2017 The role of reactive oxygen species (ROS) in the biological activities of metallic nanoparticles Int. J. Mol. Sci. 18 1-21 
Djurišić A B, Leung Y H, Ng A M C, Xu X Y, Lee P K H, Degger N and Wu R S S 2015 Toxicity of metal oxide nanoparticles: Mechanisms, characterization, and avoiding experimental artefacts Small $1126-44$

Elsässer T, Krämer M and Scholz M 2008 Accuracy of the local effect model for the prediction of biologic effects of carbon ion beams in vitro and in vivo. Int. J. Radiat. Oncol. Biol. Phys. 71 866-72 Online: http://www.ncbi.nlm.nih.gov/pubmed/18430521

Hawkins R B 2003 A microdosimetric-kinetic model for the effect of non-Poisson distribution of lethal lesions on the variation of RBE with LET. Radiat. Res. 160 61-9

Henthorn N T, Warmenhoven J W, Sotiropoulos M, Mackay R I, Kirkby K J and Merchant M J 2017 Nanodosimetric Simulation of Direct Ion-Induced DNA Damage Using Different Chromatin Geometry Models Radiat. Res. RR14755.1 Online: http://www.rrjournal.org/doi/10.1667/RR14755.1

Ionita P, Spafiu F and Ghica C 2008 Dual behavior of gold nanoparticles, as generators and scavengers for free radicals J. Mater. Sci. 43 6571-4 Online: http://www.springerlink.com/index/10.1007/s10853-008-2987-1

Jain S, Coulter J A, Hounsell A R, Butterworth K T, McMahon S J, Hyland W B, Muir M F, Dickson G R, Prise K M, Currell F J, O'Sullivan J M and Hirst D G 2010 Cell-specific Radiosensitization by Gold Nanoparticles at Megavoltage Radiation Energies Int. J. Radiat. Oncol. Biol. Phys.79 531-9 Online: http://www.ncbi.nlm.nih.gov/pubmed/21095075

Jones B L, Krishnan S and Cho S H 2010 Estimation of microscopic dose enhancement factor around gold nanoparticles by Monte Carlo calculations Med. Phys. 373809 Online: http://link.aip.org/link/?MPHYA6/37/3809/1

Kang B, Mackey M a. and El-sayed M a. 2010 Nuclear Targeting of Gold Nanoparticles in Cancer Cells Induces DNA Damage, Causing Cytokinesis Arrest and Apoptosis JACS Commun. 132 1517-9

Lampe N, Karamitros M, Breton V, Brown J M C, Kyriakou I, Sakata D, Sarramia D and Incerti S 2018 Mechanistic DNA damage simulations in Geant4-DNA part 1: A parameter study in a simplified geometry Phys. Medica 48 135-45 Online: http://dx.doi.org/10.1016/j.ejmp.2018.02.011 
Lechtman E, Mashouf S, Chattopadhyay N, Keller B M, Lai P, Cai Z, Reilly R M and Pignol J-P 2013 A Monte Carlo-based model of gold nanoparticle radiosensitization accounting for increased radiobiological effectiveness. Phys. Med. Biol. 58 3075-87 Online: http://www.ncbi.nlm.nih.gov/pubmed/23594417

Lin Y, McMahon S J, Scarpelli M, Paganetti H and Schuemann J 2014 Comparing gold nano-particle enhanced radiotherapy with protons, megavoltage photons and kilovoltage photons: a Monte Carlo simulation Phys. Med. Biol. 59 7675-89 Online: http://stacks.iop.org/00319155/59/i=24/a=7675?key=crossref.63689ea3d59c3d8f07d702726eca19b4

McMahon S J, Hyland W B, Muir M F, Coulter J A, Jain S, Butterworth K T, Schettino G, Dickson G R, Hounsell A R, O’Sullivan J M, Prise K M, Hirst D G and Currell F J 2011 Biological consequences of nanoscale energy deposition near irradiated heavy atom nanoparticles Sci. Rep. 118 Online:

http://www.nature.com/srep/2011/110620/srep00018/full/srep00018.html

McMahon S J, Mendenhall M H, Jain S and Currell F 2008 Radiotherapy in the presence of contrast agents: a general figure of merit and its application to gold nanoparticles Phys. Med. Biol. 53 5635-51 Online: http://www.ncbi.nlm.nih.gov/pubmed/18812647

McMahon S J, Schuemann J, Paganetti H and Prise K M 2016 Mechanistic Modelling of DNA Repair and Cellular Survival Following Radiation-Induced DNA Damage Sci. Rep. 633290 Online: http://www.nature.com/articles/srep33290

Meylan S, Incerti S, Karamitros M, Tang N, Bueno M, Clairand I and Villagrasa C 2017 Simulation of early DNA damage after the irradiation of a fibroblast cell nucleus using Geant4-DNA Sci. Rep. 711923 Online: http://www.nature.com/articles/s41598-01711851-4

Taleei R and Nikjoo H 2013 Biochemical DSB-repair model for mammalian cells in G1 and early S phases of the cell cycle. Mutat. Res. 756 206-12 Online: http://www.ncbi.nlm.nih.gov/pubmed/23792210

Winkler D A, Burden F R, Yan B, Weissleder R, Tassa C, Shaw S and Epa V C 2014 Modelling and predicting the biological effects of nanomaterials SAR QSAR Environ. Res. 25 161-72 Online: http://dx.doi.org/10.1080/1062936X.2013.874367 
Zheng Y and Sanche L 2009 Gold nanoparticles enhance DNA damage induced by anticancer drugs and radiation Radiat. Res. 172114 Online: http://dx.doi.org/10.1667/RR1689.1 


\title{
Molecular Mechanisms of Metal Nanoparticle
}

\section{(MNP)-Mediated Radiation Enhancement}

\author{
Karl T. Butterworth ${ }^{1}$ and Kevin M. Prise ${ }^{1}$ \\ ${ }^{1}$ Centre for Cancer Research and Cell Biology, Queen's University Belfast, Belfast, Northern Ireland, United Kingdom.
}

\section{Status}

The unique physicochemical properties of high atomic number $(Z)$ metal nanoparticles (MNPs) have made them attractive as potential radiosensitizers in cancer radiotherapy based on their strong photoelectric absorption coefficients in comparison to soft tissue. Since the seminal study by Hainfeld and colleagues [1], which demonstrated a significant increase in the survival of mice irradiated following injection of $1.9 \mathrm{~nm}$ gold nanoparticles (GNPs), a large body of preclinical evidence gained across multiple different MNPs, tumour models and radiation sources, has demonstrated the clear potential of MNPs as radiosensitizers [2,3]. However, these data are confounded as the majority of experimental observations cannot be accurately predicted from physical parameters including mass energy absorption and MNP concentration. This raises important questions concerning the underlying mechanisms of MNPmediated radiosensitization which is not primarily driven by increased total dose delivered to target cells [2].

Over the past decade, the field has made concerted efforts aiming to resolve the disparity between predictions of MNP radiosensitization and observed biological effects. This has involved detailed studies of the underlying molecular mechanisms of MNP radiosensitization for gold, silver, platinum, hafnium and gadolinium particles. Our group has largely focussed on understanding the radiobiological effects of gold nanoparticles (GNPs) in studies using model systems which have evolved from simple plasmid DNA through to cellular 
systems and in vivo tumour models (Butterworth et al 2008, Jain et al 2011, Butterworth et al 2016). For the first time, we demonstrated GNP-mediated radiosensitization at MV energies, an unexpected finding due to the dominance of Compton effects in the MV energy range which are unrelated to $Z$, and strongly implicated biologically driven radiosensitisation [5].

Subsequent reports from our laboratory and others have aimed to delineate the molecular mechanisms of MNP-mediated radiosensitization (Reviewed in Butterworth et al 2012, Rosa et al 2017). The majority of experimental reports have highlighted oxidative stress, DNA damage response and cell cycle effects as critical response mechanisms (summarised in figure 1). Furthermore, we reported that significantly elevated levels of DNA damage may be a direct result of impaired mitochondrial functional manifested by increased oxidation and loss of membrane potential [8].

Considering the broad range of experimental parameters and end-points investigated, it is difficult to draw overall conclusions on a critical mechanism of action for MNP- mediated radiosensitization. Clearly, the underlying mechanisms are the products of multiple and complex physical, chemical and biological interactions, that ultimately result in enhanced cell killing and have implications for the radiosensitivity, repair, reassortment, repopulation and reoxygenation of tumour cells [9]. Importantly, these effects need to be better resolved to improve the translation of MNPs to clinical evaluation and in realising their promise in cancer radiotherapy.

\section{Current and future challenges}

Despite a significant body of evidence demonstrating the potential of MNPs to enhance radiation response, only a very small number of agents have progressed to clinical trials. These include nànoparticle formulations of gold (CYT-6091, Cytimmune, NCT00356980; NCT00436410), gadolinium (AGuIX, NH TherAguix, NCT02820454; NCT03818386; 
NCT03308604) and hafnium oxide (NBTXR3, Nanobiotix, NCT01433068; NCT02721056; NCT02805894). Preclinical studies have provided considerable insight into the molecular mechanisms of MNP radiosensitization, yet there remains a number of considerable challenges to be addressed if the successful translation of investigative new MNPs to early phase trials is to improve.

One of the greatest challenges preventing MNPs from entering the clinic is the need for a more comprehensive delineation of the impact of their physicochemical characteristics on radiobiological response. The wide-ranging properties of MNPs that have been investigated in radiation studies prevent a general description of the underlying mechanisms of radiosensitization, which is a significant limitation in optimising MNP-based therapy. We have begun to understand the influences of element, size, shape, surface modification and source energy. As an example, our group demonstrated the thiol coating on a commonly used commercial $1.9 \mathrm{~nm}$ GNP to significantly modulate cellular pathways relating to oxidative stress, and inhibition of this effect almost entirely abrogated the radiosensitizing effect of the GNP [10]. A precise understanding of how these variables control key biological interactions and cellular localisation is required to inform the optimum physical parameters of MNPs, but as of yet these have not been systemically investigated in standardised cell models.

Considering nuclear DNA as the critical target of radiation induced damage, it would be expected that the subcellular localisation of MNPs would have a major impact on radiobiological response. In particular, it may be expected that proximity to the nucleus is critical due to the short range of Auger electrons emitted which cause a series of clustered ionising events around the nanoparticle [11]. Using a soft X-ray microbeam targeting nuclear or cytoplasmic cellular compartments, our group demonstrated that even in the absence of nuclear localisation, cytoplasmic irradiation can drive significant DNA damage, again identifying the mitochondria as a central mediator in GNPs radiosensitization [12]. These data 
raise intriguing questions concerning subcellular localisation and critical organelle targets for MNP effects, which will require more sophisticated imaging methods if they are to be better understood.

Finally, most MNPs rely on the enhance permeability retention (EPR) effect for tumour specificity due to abnormal vasculature. The development of improved methods for tumour targeting has the potential to make a significant impact and lead to the application of MNPs for precision medicine. For example, HER-2 targeted MNPs for use in breast cancer have been postulated to have potential [13], but to date none of the many potential approaches in this area have translated to the clinic.

\section{Recent advances in science and technology to meet challenges}

The future success of MNPs in the clinic is predicated on a more detailed mechanistic understanding of the impact of key physical variables on cellular interactions and how these determine cellular radiobiological response. As frequently occurs, technologic evolutions lead to transformative impacts on scientific research, which will continue to propagate discovery in the MNP research field.

Recent advances in gene expression technologies such as single cell sequencing allow for studies of the genomic and transcriptomic landscape of tumours at the single cell level [14]. This approach could provide critical insight in the multicellular complexity of MNP radiosensitization along with spatial information about tissue context or volumetric localization of gene expression changes. Advanced gene and protein-based methods may be used to characterise alterations in immune cell types and improve understanding of interactions between MNPs, the immune system and tumours. In the immunotherapy era, this approach may be central to identifying novel opportunities for combining MNPs with immune-oncology 
agents. Finally, these approaches could also identify biomarkers for the selection of patients most likely to respond to MNP enhanced radiotherapy.

There is also a need to apply improved preclinical models to demonstrate the radiobiological effects of MNPs in tumours and normal tissues. Approaches could include the use of standardised cell models, syngeneic transplants or patient derived tumour models established in humanised host animals. Tumour response data should be supported by evaluation of MNP effects in disease relevant organs at risk, all of which should be evaluated using precision small animal image guided radiotherapy [15]. These developments are likely to synergise with new tools for improved theoretical modelling of MNPs utilising relevant, systematic, mechanistic models and enabling the integration of many heterogeneous datasets to produce useful predictions for a range of different MNP. The integration of these approaches will undoubtedly improve our understanding of the fundamental processes underlying MNP radiosensitization, leading to improved, biologically optimised treatments.

\section{Concluding remarks}

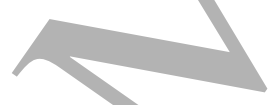

Technological advances and improved understanding of mechanisms of cellular radiobiological response will continue to play critical roles in improving the efficacy of radiotherapy. MNP agents have high potential to increase the therapeutic index and impact standard of care in clinical oncology, yet there remain critical challenges towards clinical translation. Addressing these challenges requires a multidisciplinary strategy focused on applying state-of-the-art technologies to better understand the physical, chemical and biological basis of MNP radiosensitization, and ultimately deliver optimised therapies to the clinic. 
A

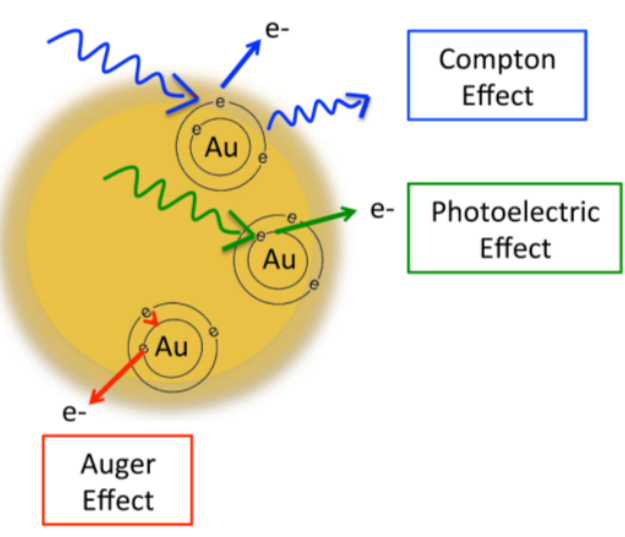

B

Molecular mechanisms of MNP radiation enhancement
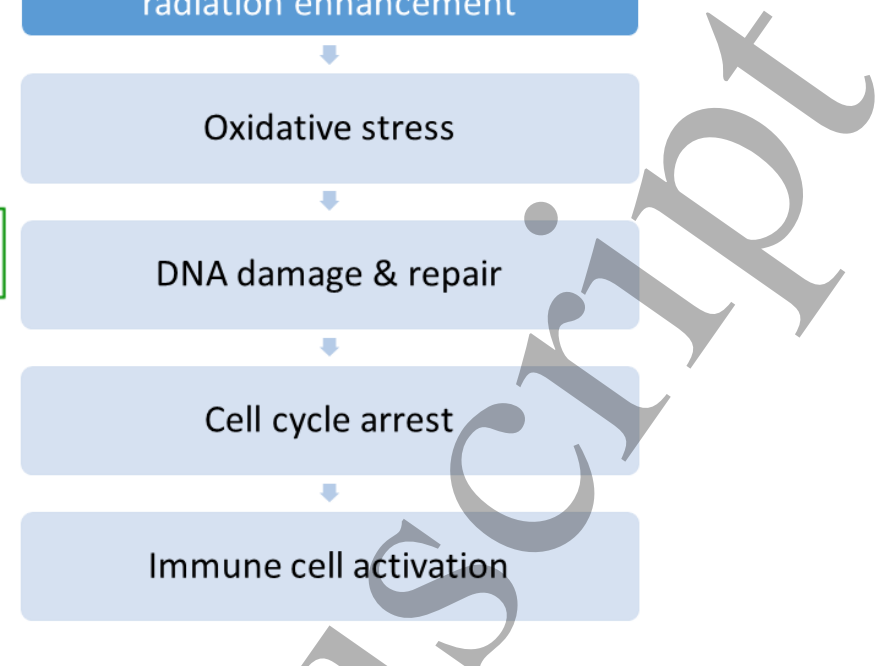

Figure 1. Schematic illustration of the physics processes (panel A) and molecular mechanisms (panel B) of MNP-mediated radiation enhancement. 


\section{References}

[1] Hainfeld JF, Slatkin DN, Smilowitz HM. The use of gold nanoparticles to enhance radiotherapy in mice. Phys Med Biol 2004;49. doi:10.1088/0031-9155/49/18/N03.

[2] Butterworth KTT, McMahon SJJ, Currell FJJ, Prise KMM. Physical basis and biological mechanisms of gold nanoparticle radiosensitization. Nanoscale 2012;4:4830-8. doi:10.1039/c2nr31227a.

[3] Haume K, Rosa S, Grellet S, Śmiałek MAMA, Butterworth KT, Solov'yov AVAV, et al. Gold nanoparticles for cancer radiotherapy: a review. Cancer Nanotechnol 2016;7:8. doi:10.1186/s 12645-016-0021-x.

[4] Butterworth KT, Wyer JA, Brennan-Fournet M, Latimer CJ, Shah MBB, Currell FJ, et al. Variation of strand break yield for plasmid DNA irradiated with high-Z metal nanoparticles. Radiat Res 2008;170:381-7. doi:10.1667/RR1320.1.

[5] Jain S, Coulter JA, Hounsell AR, Butterworth KT, McMahon SJ, Hyland WB, et al. Cell-Specific Radiosensitization by gold nanoparticles at megavoltage radiation energies. Int J Radiat Oncol Biol Phys 2011;79. doi:10.1016/j.ijrobp.2010.08.044.

[6] Butterworth, K. T., Nicol, J. R., Ghita, M., Rosa, S., Chaudhary, P., McGarry, C. K., ... Prise KM, Butterworth KT, Nicol JR, Ghita M, Rosa S, Chaudhary P, et al. Preclincial evaluation of Gold-DTDTPA Nanoparticles As Theranostic Agents In Prostate Cancer Radiotherapy. Nanomedicine 2016;10.2217/nn:2035-47. doi:10.2217/nnm-2016-0062.

[7] Rosa S, Connolly C, Schettino G, Butterworth KT, Prise KM. Biological mechanisms of gold nanoparticle radiosensitization. Cancer Nanotechnol 2017;8. doi:10.1186/s12645-017-0026-0.

[8] Taggart LELE, McMahon SJSJ, Currell FJFJ, Prise KMKM, Butterworth KTKT. The role of mitochondrial function in gold nanoparticle mediated radiosensitisation. Cancer Nanotechnol 2014;5:5. doi:10.1186/s12645-014-0005-7. 
[9] Cui L, Her S, Borst GR, Bristow RG, Jaffray DA, Allen C. Radiosensitization by gold nanoparticles: Will they ever make it to the clinic? Radiother Oncol 2017;124:344-56. doi:10.1016/j.radonc.2017.07.007.

[10] Taggart LE, McMahon SJ, Butterworth KT, Currell FJ, Schettino G, Prise KM. Protein disulphide isomerase as a target for nanoparticle-mediated sensitisation of cancer cells to radiation. Nanotechnology 2016;27. doi:10.1088/0957-4484/27/21/215101.

[11] McMahon SJSJ, Hyland WBWB, Muir MFMF, Coulter JAJ a, Jain S, Butterworth KTKT, et al. Biological consequences of nanoscale energy deposition near irradiated heavy atom nanoparticles. Sci Rep 2011;1:18. doi:10.1038/srep00018.

[12] Ghita M, McMahon SJ, Taggart LE, Butterworth KT, Schettino G, Prise KM. A mechanistic study of gold nanoparticle radiosensitisation/using targeted microbeam irradiation. Sci Rep 2017;7. doi:10.1038/srep44752.

[13] Reilly RM, Chattopadhyay N, Cai ZL, Pignol JP, Keller B, Lechtman E, et al. Design and Characterization of HER-2-Targeted Gold Nanoparticles for Enhanced X-radiation Treatment of Locally Advanced Breast Cancer. Mol Pharm 2010;7:2194-206. doi:10.1021/mp100207t.

[14] Chen H, Ye F, Guo G. Revolutionizing immunology with single-cell RNA sequencing. Cell Mol Immunol 2019;16:242-9. doi:10.1038/s41423-019-0214-4.

[15] Butterworth KT, Evolution of the Supermodel: Progress in Modelling Radiotherapy Response in Mice. Clin Oncol 2019;31:272-82. doi:10.1016/j.clon.2019.02.008. 


\title{
Optimization of GNP-based Cancer Therapeutics
}

\author{
B. Devika Chithrani, Kyle Bromma \\ Department of Physics and Astronomy, University of Victoria, Victoria, BC, Canada; devikac@uvic.ca
}

$\underline{\text { Status }}$

Over 20 years ago, it was demonstrated that iodine is a radiosensitizer of cells in culture[1]. It was also found that direct injection of iodine into tumors followed by radiotherapy (RT) suppressed the growth of $80 \%$ of tumors[2]. More recently, gold has been pursued as a radiosensitizer due to its higher atomic number compared to iodine $\left(Z_{\mathrm{Au}}=79 \mathrm{vs}\right.$. $\left.Z_{\mathrm{I}}=53\right)$ and favorable biocompatibility[3]. The high atomic number of gold nanoparticles (GNPs) means that they can enhance the effective radiation dose at the site of disease. During radiation therapy, the presence of GNPs enhances the cross section of low-energy electrons and other cell damaging species leading to increased cell/death[4,5]. This strategy is already being used by many groups to develop novel cancer treatments and the initial results are extremely promising[6]. The ultimate goal of these GNP-based platforms will be the targeted delivery of tumors using radiotherapy while causing minimal side effects to normal tissue.

\section{Current and future challenges}

Irradiation with kilovolt X-rays following intravenous administration of $1.9 \mathrm{~nm}$ GNPs to mice bearing subcutaneous tumors was the first study to show an increased radiosensitization effect in tumors[3]. A comparison of the mass concentrations of iodine and gold across different studies suggests that in the absence of intracellular uptake, in vivo intra-tumoral concentrations of $\sim 5 \frac{\mathrm{mg}}{\mathrm{g}}$ were required for radiosensitization[7]. In contrast, whenever GNPs were internalized in vitro, radiosensitization was achievable at concentrations as low as $1 \frac{\mathrm{ng}}{\mathrm{g}}$ [8]. Based on these recent observations, the current challenge is to achieve radiosensitization effects at dramatically lower concentrations through 
intracellular localization of GNPs within the solid tumor issue.

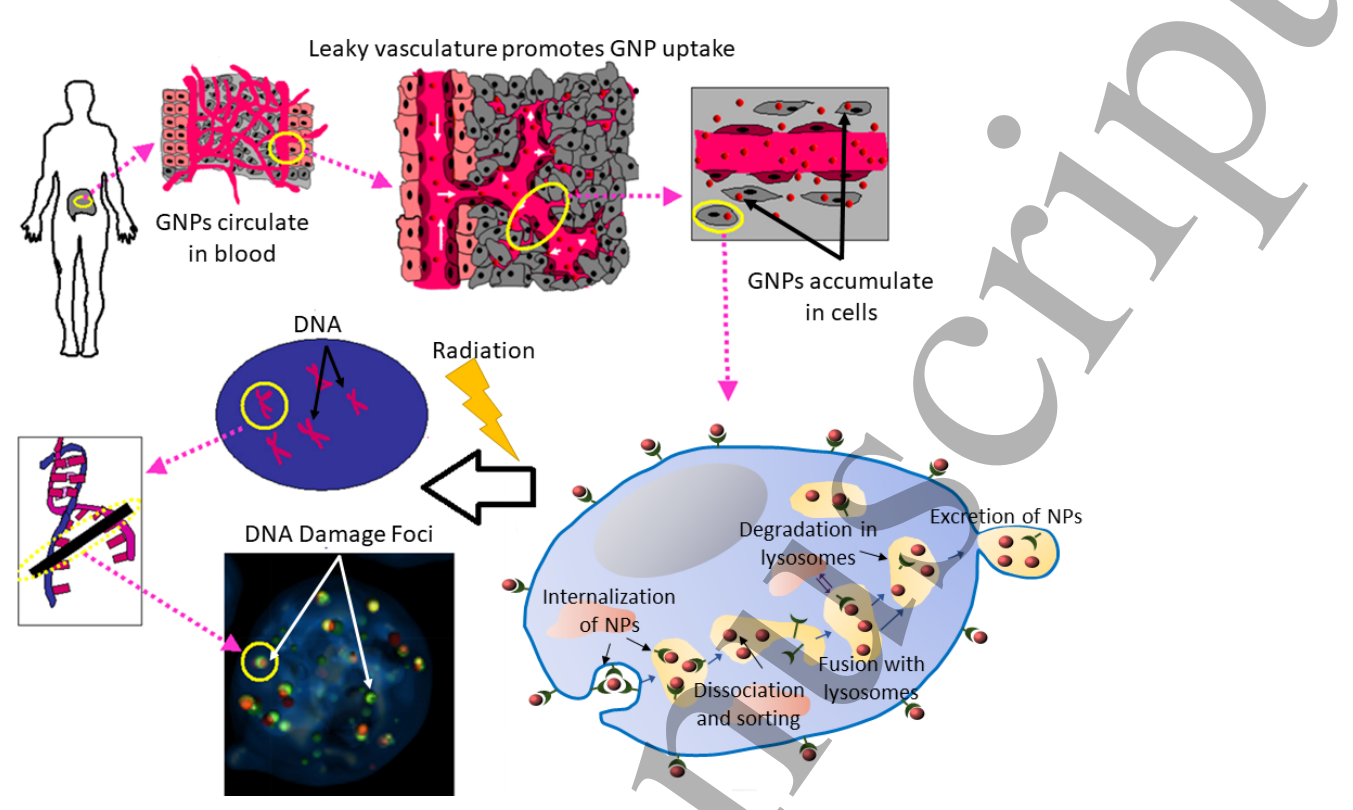

Figure 1. Roadmap to optimizing GNP-mediated radiation response. Accumulation of a higher percent of injected NP dose within the tumor cells involves enhanced blood circulation of NPs, penetration within the tumor tissue once they leave the leaky tumor blood vessels, and finally internalization of GNPs within every tumor cell. The ultimate goal is to enhance the DNA damage using GNPs as a radiosensitization agent.

\section{Advances in science and technology to meet the challenges}

Advances in building novel lipid-based platforms for efficient intracellular delivery of GNPs has shown remarkable results[9]. This can also be achieved through optimizing physicochemical properties of GNPs for individual particle delivery[10]. Successful delivery of GNPs into the intracellular space of individual cells also depends also on the efficiency of crossing a few boundaries, including tumor blood vessels to tumor tissue followed by their penetration successfully through the tumor tissue as illustrated in Figure 1. A tumor with a well-developed collagen network or extracellular matrix (ECM) can be considered to be physically resistant to NP-based therapies[11, 12]. For tumors with a strong ECM, treatments that reverse or inhibit collagen production and assembly could be performed prior to NP-based therapies [11]. Hence, downregulation of ECM may become a promising adjuvant therapeutic strategy for ECM-rich tumors [12]. Another approach could be to use ECM-degrading enzymes to modify the collagen structure to further improve the distribution of NPs in solid tumors. 
Features of solid cancers not accounted for by three-dimensional (3D) in vitro models are variable interstitial fluid pressure (IFP), the influence of convection, and stromal cells[13]. Although direct in vivo assessment has the advantage of duplicating the clinical environment more closely, these 3D in vitro models offer the advantage of being able to examine and evaluate isolated results with less complicated parameters[14]. As illustrated in Figure 2, a recent study has shown that the size and surface functionality of the NPs could be tailored using these 3D in vitro models to produce a higher accumulation within the tumor in an in vivo tumor model[15].

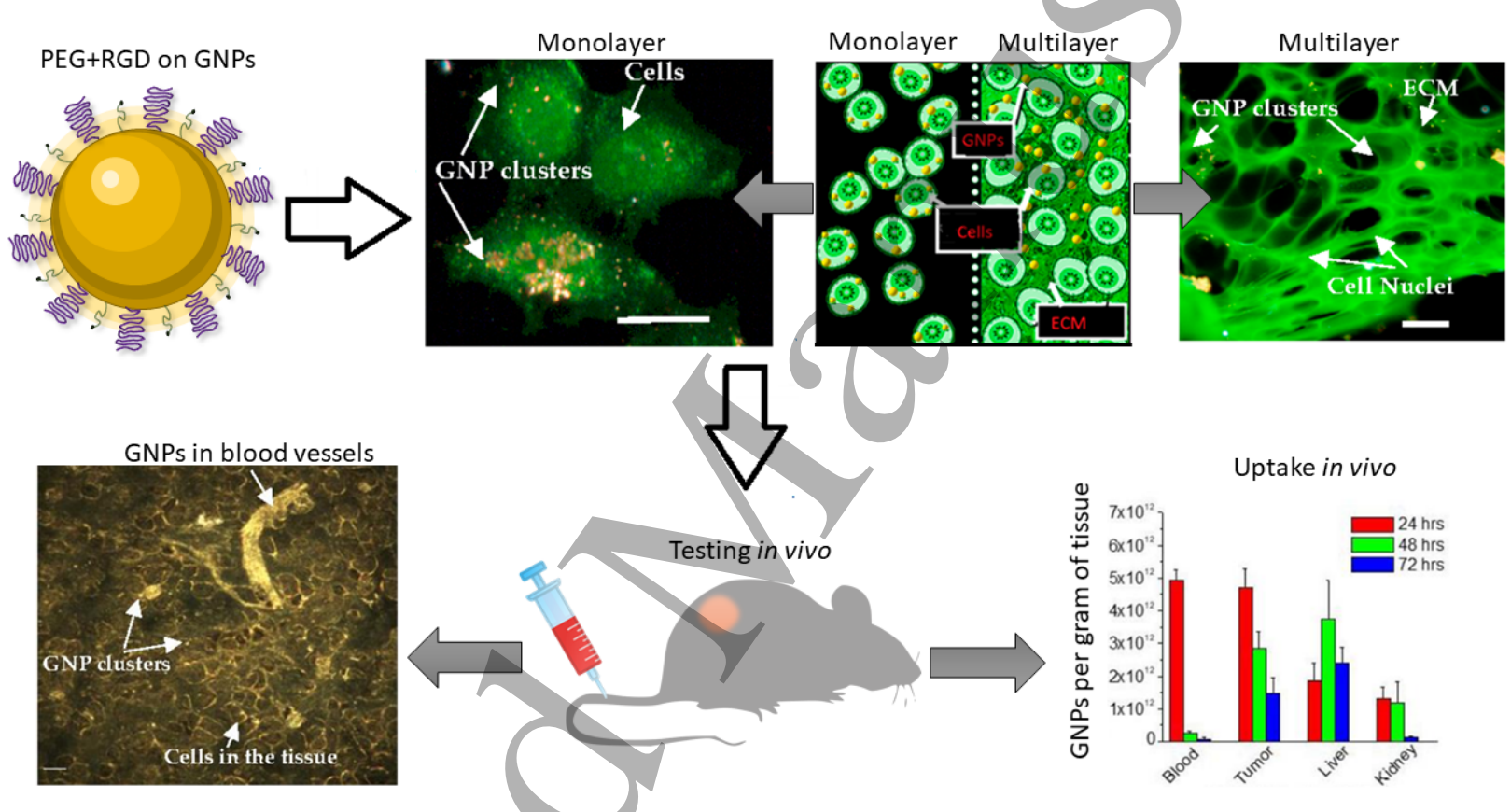

Figure 2. Optimization of NP-based delivery systems at monolayer and tissue-like multilayer models leads to their successful accumulation in in vivo models.

Radiation therapy, in particular, damages cancer cells with a less pronounced effect on the vasculature[16]. It has been shown that nano-sized molecules enter radiation-treated tumors at a rate 2.2-fold higher than non-irradiated tumors[17]. Radiation killed well-oxygenated cancer cells near tumor blood vessels leading to higher vascular permeability[16]. However, excessive radiation damage to blood vessels can shut down blood flow, which affected the nano-therapeutic agent delivery negatively. Moreover, the effects of radiation on the ECM must be considered since radiation could damage the long polymer chains of hyaluronan and elastin resulting in premature stiffening of tissue[18]. Hence, the effect of radiation on the ECM and tumor blood vessels should be taken into account when designing NP-based 


\section{$\underline{\text { References }}$}

The incorporation of cancer nanomedicine into current therapeutic protocols requires the careful consideration of multiple factors to overcome the barriers for intracellular NP delivery within the tumor. Three-dimensional tumor models could be further improved to test NP-based therapeutics in parallel to animal models to improve our understanding and overcome barriers to NP transport while understanding the mechanisms of tumor cell death in a less complex tumor microenvironment. Introduction of GNPS to current chemoradiation protocols could potentially be another addition to the current toolbox that could benefit some patients[20]. Most of the in vitro and in vivo data have shown promising results with clinically relevant MV photon beams and lower concentration of GNPs. Hence, clinical translation of GNP-mediated radiosensitization could be achieved with a collective effort from research groups around the world.

[1] H. Matsudaira, A.M. Ueno, I. Furuno, lodine Contrast Medium Sensitizes Cultured Mammalian Cells to X Rays but Not to X Rays, Radiat Res, 84 (1980) 144-148.

[2] R. Santos Mello, H. Callisen, J. Winter, A.R. Kagan, A. Norman, Radiation dose enhancement in tumors with iodine, Med Phys, 10 (1983) 75-78.

[3] J.F. Hainfeld, D.N. Slatkin, H.M. Smilowitz, The use of gold nanoparticles to enhance radiotherapy in mice, Phys Med Biol, 49 (2004) N309-315.

[4] Y. Zheng, L. Sanche, Low Energy Electrons in Nanoscale Radiation Physics: Relationship to Radiosensitization and Chemoradiation Therapy, Rev. Nanosci. Nanotechnol., 2 (2013) 1-28. [5] J.D. Carter, N.N. Cheng, Y. Qu, G.D. Suarez, T. Guo, Nanoscale Energy Deposition by X-ray Absorbing Nanostructures, J. Phys. Chem. B, 111 (2007) 11622-11625.

[6] J. Schuemann, R. Berbeco, D.B. Chithrani, S.H. Cho, R. Kumar, S.J. McMahon, S. Sridhar, S. Krishnan, Roadmap to Clinical Use of Gold Nanoparticles for Radiation Sensitization, International Journal of Radiation Oncology • Biology • Physics, 94 (2016) 189-205. 
[7] J.H. Rose, A. Norman, M. Ingram, C. Aoki, T. Solberg, A. Mesa, First radiotherapy of human metastatic brain tumors delivered by a computerized tomography scanner (CTRx), Int J Radiat Oncol Biol Phys, 45 (1999) 1127-1132.

[8] X. Zhang, J.Z. Xing, J. Chen, L. Ko, J. Amanie, S. Gulavita, N. Pervez, D. Yee, R. Moore, W. Roa, Enhanced radiation sensitivity in prostate cancer by gold-nanoparticles, Clin Invest Med, 31 (2008) E160167.

[9] K. Bromma, K. Rieck, J. Kulkarni, C. O’Sullivan, W. Sung, P. Cullis, J. Schuemann, D.B. Chithrani, Use of a lipid nanoparticle system as a Trojan horse in delivery of gold nanoparticles to human breast cancer cells for improved outcomes in radiation therapy, Cancer Nanotechnology, 10 (2019) 1.

[10] J.M. Bergen, Gold Nanoparticles as a Versatile Platform for Optimizing Physicochemical Parameters for Targeted Drug Delivery, Macromolecular bioscience, 6 506-516.

[11] P.A.B. Netti, David A.; Swartz, Melody A.; Grodzinsky, Alan J.; Jain, Rakesh K., Role of extracellular matrix assembly in interstitial transport in sold tumors, Cancer Research, 60 (2000) 2497-2503. [12] T.L. Ji, Jiayan; Wang, Jing; Cai, Rong; Zhang, Yinlong; Qi, Feifei; Zhang, Lijing; Zhao, Xiao; Wu, Wenjing; Hao, Jihui; Qin, Zhihai; Zhao, Ying; Nie, Guangjun, Designing liposomes to suppress extraceullular matrix expression to enhance drug penetration and pancreatic tumor therapy, ACS nano, 11 (2017) 8668-8678.

[13] A.I.T. Minchinton, lan F., Drug penetration in solid tumours, Nature Reviews Cancer, 6 (2006) 583592.

[14] D. Yohan, C. Cruje, X. Lu, B.D. Chithrani, Size Dependent Gold Nanoparticle Interaction at NanoMicro Interface Using both Monolayer and Multilayer (Tissue-Like) Cell Models, Nano-Micro Lett., 8 (2016) 44-53.

[15] C. Yang, K. Bromma, B.D. Chithrani, Peptide Mediated In Vivo Tumor Targeting of Nanoparticles through Optimization in Single and Multilayer In Vitro Cell Models, Cancers, 10 (2018) 84.

[16] Y.M. Nakamura, Ai; Choyke, Peter L.; Kobayashi, Hisataka, Nanodrug Delivery: Is the Enhanced Permeability and Retention Effect Sufficient for Curing Cancer, Bioconjugate chemistry, 27 (2016) 22252238.

[17] H.R. Kobayashi, Koen; English, Sean; Yordanov, Alexander T.; Milenic, Diane E.; Sowers, Anastasia L.; Citrin, Deborah; Krishna, Murali C.; Waldmann, Thomas A.; Mitchell, James B.; Brechbiel, Martin W., Application of a macromolecular contrast agent for detection of alteration of tumor vessel permeability induced by radiation, Clinical cancer research, 10 (2004) 7712-7720. 
[18] F.B. Mohamed, D.A.; Winlove, C.P., Effects of ionizing radiation on extracellular matrix, Nuclear Instruments and Methods in Physics Research Section A, 580 (2007) 566-569.

[19] R.T.B. Tong, Yves; Kozin, Sergey V.; Winkler, Frank; Hicklin, Daniel J.; Jain, Rakesh K., Vascular normalization by vascular endothelial growth factor receptor 2 blockade induces a pressure gradient across the vasculature and improves drug penetration in tumors Cancer Research, 64 (2004) 3731-3736. [20] S. Jelveh, D.B. Chithrani, Gold Nanostructures as a Platform for Combinational Therapy in Future Cancer Therapeutics, Cancers, 3 (2011) 1081-1110. 


\section{Targeting Tumor Vasculature with Nanoparticles for}

\section{Radiation Therapy}

Needa Virani*, Ross Berbeco*, Sijumon Kunjachan*

Department of Radiation Oncology, Brigham and Women's Hospital, Dana-Farber Cancer Institute and Harvard Medical School, Boston, MA USA

*all authors contributed equally to this work

\section{Status}

Limitations of some nanoparticle-based cancer therapies include inadequate diffusion into the tumor interior and poor tissue penetration of stimulating agents (optical, IR, UV, kV x-rays, etc.). While relying solely on the inherently leaky tumor vasculature limits nanoparticle delivery to tumor cells, this challenge can be turned into an advantage by targeting tumor vasculature. Nanoparticles of a certain size tend to be trapped in tumor blood vessels and nanoparticle design can further enhance vascular accumulation. Situations in which passive tumor targeting confronts various physiological barriers (e.g. vascular walls, tissue, collagen, ECM matrix, etc.), nanoparticles actively targeted to tumor blood vessels have direct access to the therapeutic target. Several peptides have been introduced to actively target tumor endothelial cell receptor expression, increasing accumulation in these sites. For example, Arginine-Glycine-Aspartic Acid (RGD) has been shown to increase the nanoparticle concentration in and near tumor endothelial cells (1) (Figure 1).

Tumor endothelial cells provide reliable receptor expression compared to other tumor cellular targets. In cancer cells, receptor
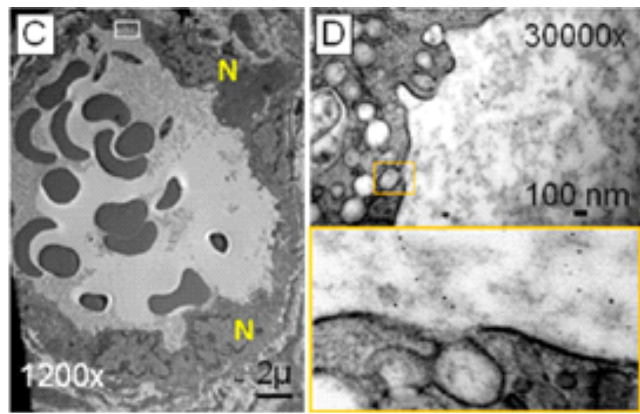

Figure 1. TEM imaging of tumor endothelial cell uptake in a mouse tumor model. Vascular targeted gold nanoparticles are shown in tumor endothelium $1 \mathrm{~h}$ after IV administration. Magnified images show uptake via clathrin/caveolae vesicles. Reprinted with permission from (Kunjachan Nano Letters 2015). Copyright 2015 American Chemical Society. expressions and density can vary depending on the tumor type, origin, stage, and mutational characteristics. All these factors make tumor endothelial targeting a more consistent approach across different disease sites. In addition, the range of the local radiation dose enhancement - and subsequent biological damage - via the production of low energy electrons (2) is relatively low (several microns), meaning that nanoparticles need to be close to the target cells. Therefore, vascular targeted nanoparticles will confer major damage to tumor blood vessels after irradiation. 
There is strong evidence that tumor vasculature plays an important role in radiation therapy (3-5). Garcia-Barros et al. proposed that damage to tumor vasculature during radiation therapy may be more therapeutically beneficial than clonogenic cell death (3). A review by Park et al. presented experimental evidence linking radiation-induced tumor vascular damage to the success of stereotactic body radiation therapy (SBRT) procedures (5). Similarly, vascular disruption of hepatocellular carcinoma with injected Y-90 microspheres is a proven clinical technique (6).

The concept of targeting tumor vasculature with nanoparticles to amplify radiation dose for vascular disruption was first proposed by Berbeco et al.(7). It has since been demonstrated that radiation therapy combined with vascular-targeted gold nanoparticles destroys tumor endothelial cells while largely sparing normal tissues (8, 20). In that work, gold nanoparticles were targeted to tumor blood vessels via RGD prior to radiation therapy. Imaging and histology assays demonstrated the endothelial cell damage and subsequent vascular disruption (Figure 2). This group also demonstrated increased model nanodrug delivery after vessel disruption, suggesting a benefit for combined therapies (20).

Vascular therapies improve the effects of radiation therapy (9). Preclinical experimental results indicate a synergistic relationship between vascular disruption and radiation therapy (10). However, no clinical trials have yet been reported with chemical vascular disrupting agents (VDAs) and radiation therapy. Negative results of a Phase 3 trial of chemical VDAs with chemotherapy (11) have been attributed to clinical factors (12). Using targeted GNPs rather than chemical VDAs should reduce off-target toxicities because GNPs are inactive outside the high radiation volume.
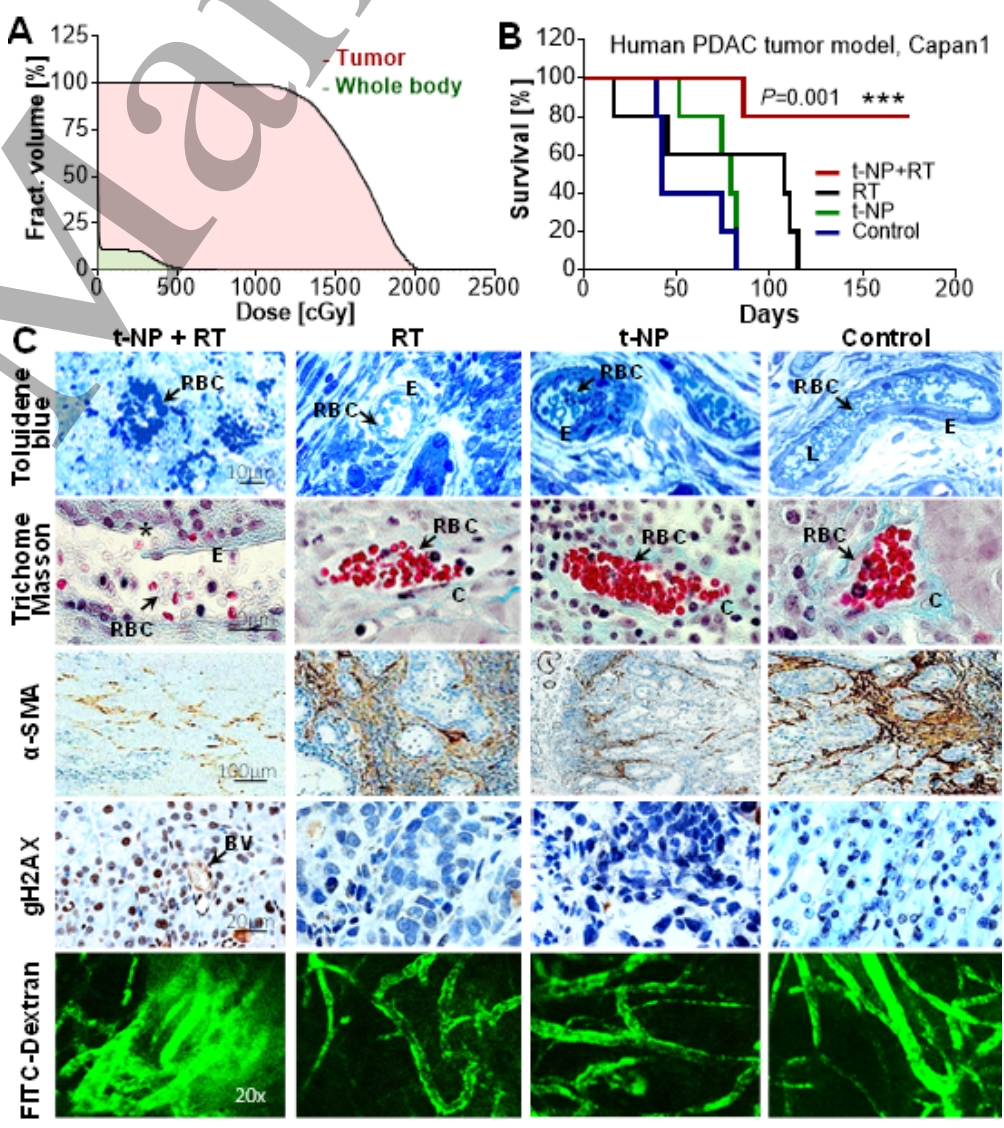

Figure 2. Vascular disruption after delivery of targeted gold nanoparticles and radiation therapy. Endothelial destruction is observed via histopathology and confocal microscopy.

\section{Current and Future Challenges} (from Kunjachan Scientific Reports 2019)

Changes in tumor physiology after vascular disruption may present both benefits and challenges for radiation therapy. For example, tumor vascular disruption may increase hypoxia. Hypoxic tumor regions represent 
resistant cell populations requiring additional therapeutic attention and failure to address hypoxia has implications for tumor therapy response as well as metastatic potential. The hypoxic conditions that are associated with tumors create a cellular response in which alternative metabolic pathways are enabled by factors such as HIF-1 $\alpha$ which has been shown to be an exclusive regulator of carbonic anhydrase 9 (CA IX) activation. CA IX is largely absent from normal tissues and its over-expression is a clinical biomarker for poor prognosis (13).

The tissue penetration of high energy (MV) photons is an advantage of external beam radiation therapy (EBRT) as a nanoparticle "activating" agent. These photons have significant skin sparing properties and deliver $>60 \%$ of their maximum dose at $10 \mathrm{~cm}$ depth, for example. However, the penetration benefits of the high energy beam are somewhat mitigated by the physical interaction properties. The probability of a photoelectric interaction between an atom in the nanoparticle and an incident photon is proportional to $1 / E^{3}$, where $E$ is the energy of the incident photon. EBRT beams are composed of a spectrum of photon energies peaking well outside the range of photoelectric interactions (e.g. $6 \mathrm{MV})$. For this reason, combining nanoparticles with EBRT has been dismissed as impractical(14). However, roughly 95\% of clinical radiation therapy procedures utilize this energy range, therefore a solution is necessary for nanoparticles to be clinically translated.

\section{Advances in Science and Technology to Meet Challenges}

The advancement of non-invasive hypoxia imaging will provide an opportunity to study temporary and permanent physiological changes within the tumor microenvironment to better understand the effects of nanoparticle targeted vascular disruption therapies. Current modalities for hypoxia identification include oxygen electrodes for direct measurement of $\mathrm{pO}_{2}$ levels, exogenous markers such as $\mathrm{HIF-1} \alpha$, and imaging modalities such as positron emission tomography (PET) and magnetic resonance imaging (MRI). Although oxygen electrodes provide a direct $\mathrm{pO}_{2}$ measurement, readings are limited to invasive, point measurements. HIF-1 $\alpha$ and pimonidazole are used currently in clinical practice, however the results are limited to the area of the biopsy. PET imaging with hypoxía specific radiotracers such as [ $\left.{ }^{18} \mathrm{~F}\right]$ labeled misonidazole (FMISO) is noninvasive but is limited due to the cost as well as permeation limitations of small molecules. In the preclinical setting, there are additional optical imaging methods to image CA IX expression in vivo(15). An emerging noninvasive modality is functional MRI based diffusion-weighted (DWI) and blood/tissue oxygen level dependent (BOLD/TOLD) imaging. Both use gradient echo pulses to image the relative paramagnetic properties of oxygenated/and deoxygenated hemoglobin within the whole tumor (16). Advantages of MRI-based approaches include non-invasive, serial imaging capabilities and translational potential to human scales. 
Once the time-course of induced hypoxia has been identified, new techniques can be used to mitigate the therapeutic impact. Identification of these regions has begun transitioning cancer therapy towards improving tissue oxygenation. Current clinical trials are underway studying the use of hypoxia-activated prodrugs'such as tirapazamine, hyperbaric chambers, and radiation dose painting based on hypoxia maps. Other strategies include increasing hypoxic cell radiosensitivity via misonidazole, decreasing oxygen consumption via metformin, and specifically targeting hypoxia surface markers such as HIF-1 $\alpha$ (17).

There is ample evidence that disrupting tumor blood vessels can increase drug delivery, improving outcomes (18). Ultrasound stimulated microbubbles (USMB) is a well-researched preclinical approach and has resulted in positive clinical results of a Phase 1 trial (19). USMB vascular modulation is limited, however, as it is not appropriate in all parts of the body. Published work with vascular-targeted gold nanoparticles demonstrated increased perfusion of an injected dye after disruption (20) (Figure 2) and further quantification by optical and MR imaging showed increased permeability and accumulation of model nanodrugs (20). Recent advances in drug nanoformulations will improve tumor delivery, particularly after vascular disruption.

While EBRT beams peak at high energies, they also contain substantial contributions of low energy photons. Key radiation treatment planning parameters will also affect the proportion of low energy photons at the site of disease (21). At the patient surface, the fraction of low energy $(<150 \mathrm{kV})$ photons is small $(0.5 \%)$. Due to scattering processes, this increases to $8 \%$ at $10 \mathrm{~cm}$ depth and $11 \%$ for a flattening filter free (FFF) beam. Additional modifications of the linear accelerator can be made to further increase the low energy photon content without compromising the overall dose conformity (22).

\section{Concluding Remarks}

Recent studies have demonstrated the therapeutic benefit of targeting tumor blood vessels with nanoparticles prior to radiation therapy. Induced hypoxia after vascular disruption needs to be considered and mitigating actions should be included if needed. While preliminary results indicate that the subsequent vascular disruption will increase model drug delivery, careful studies in advanced translational models should be undertaken to fully understand the combined impact on tumor growth. The tools for these steps are currently available and therefore it is anticipated that clinical translation of this therapeutic approach will follow. 


\section{References}

1. Kunjachan S, Pola R, Gremse F, Theek B, Ehling J, Moeckel D, Hermanns-Sachweh B, Pechar M, Ulbrich K, Hennink WE. Passive versus active tumor targeting using RGD-and NGR-modified polymeric nanomedicines. Nano letters. 2014;14(2):972-81.

2. Hainfeld JF, Slatkin DN, Smilowitz HM. The use of gold nanoparticles to enhance radiotherapy in mice. Physics in Medicine and Biology. 2004;49(18):N309-N15. PubMed PMID: ISI:000224395600019.

3. Garcia-Barros M, Paris F, Cordon-Cardo C, Lyden D, Rafii S, Haimovitz-Friedman A, Fuks Z, Kolesnick R. Tumor response to radiotherapy regulated by endothelial cell apoptosis. Science (New York, NY). 2003;300(5622):1155-9. PubMed PMID: ISI:000182886500049.

4. Fuks Z, Kolesnick R. Engaging the vascular component of the tumor response. Cancer Cell. 2005;8(2):8991. PubMed PMID: ISI:000231330100001.

5. Park HJ, Griffin RJ, Hui S, Levitt SH, Song CW. Radiation-induced vascular damage in tumors: implications of vascular damage in ablative hypofractionated radiotherapy (SBRT and SRS). Radiat Res. 2012;177(3):311-27. Epub 2012/01/11. PubMed PMID: 22229487.

6. Edeline J, Gilabert M, Garin E, Boucher E, Raoul JL. Yttrium-90 microsphere radioembolization for hepatocellular carcinoma. Liver cancer. 2015;4(1):16-25. Epub 2015/05/29. doi: 10.1159/000343878. PubMed PMID: 26020026; PMCID: Pmc4439788.

7. Berbeco RI, Ngwa W, Makrigiorgos GM. Localized dose enhancement to tumor blood vessel endothelial cells via megavoltage x-rays and targeted gold nanoparticles: new potential for external beam radiotherapy. International Journal of Radiation Oncology Biology Physics. 2011;81(1):270-6. doi: 10.1016/j.ijrobp.2010.10.022. PúbMed PMID: WOS:000294093300037.

8. Kunjachan S, Detappe A, Kumar R, Ireland T, Cameron L, Biancur DE, Motto-Ros V, Sancey L, Sridhar S, Makrigiorgos GM, Berbeco RI. Nanoparticle Mediated Tumor Vascular Disruption: A Novel Strategy in Radiation Therapy. Nano Lett. 2015;15(11):7488-96. Epub 2015/09/30. doi: 10.1021/acs.nanolett.5b03073. PubMed PMID: 26418302; PMCID: PMC5507193

9. Siemann DW, Rojiani AM. The vascular disrupting agent ZD6126 shows increased antitumor efficacy and enhanced radiation response in large, advanced tumors. International Journal of Radiation Oncology Biology Physics. 2005;62(3):846-53. doi: 10.1016/j.ijrpbp.2004.02.048. PubMed PMID: WOS:000229766300035.

10. Wachsberger P, Burd R, Dicker AP. Tumor response to ionizing radiation combined with antiangiogenesis or vascular targeting agents: Exploring mechanisms of interaction. Clinical Cancer Research. 2003;9(6):1957-71. PubMed PMID: ISI:000183386000002. 
11. Lara PN, Jr., Douillard JY, Nakagawa K, von Pawel J, McKeage MJ, Albert I, Losonczy G, Reck M, Heo DS, Fan X, Fandi A, Scagliotti G. Randomized phase III placebo-controlled trial of carboplatin and paclitaxel with or without the vascular disrupting agent vadimezan (ASA404) in advanced non-small-cell lung cancer. Journal of clinical oncology : official journal of the American Society of Clinical Oncology. 2011;29(22):2965-71. Epub 2011/06/29. doi: 10.1200/jco.2011.35.0660. PubMed PMID: 21709202.

12. Lorusso PM, Boerner SA, Hunsberger S. Clinical development of vascular disrupting agents: what lessons can we learn from ASA404? Journal of clinical oncology : official journal of the American Society of Clinical Oncology. 2011;29(22):2952-5. Epub 2011/06/29. doi: 10.1200/jco.2011.36.1311. PubMed PMID: 21709201.

13. Le QT, Chen E, Salim A, Cao H, Kong CS, Whyte R, Donington J, Cannon W, Wakelee H, Tibshirani R, Mitchell JD, Richardson D, O'Byrne KJ, Koong AC, Giaccia AJ. An evaluation of tumor oxygenation and gene expression in patients with early stage non-small cell lung cancers. Clinical cancer research : an official journal of the American Association for Cancer Research. 2006;12(5):1507-14. Epub 2006/03/15. doi: 10.1158/10780432.ccr-05-2049. PubMed PMID: 16533775.

14. Lechtman E, Chattopadhyay N, Cai Z, Mashouf S, Reilly R, Pignol JP. Implications on clinical scenario of gold nanoparticle radiosensitization in regards to photon energy, nanoparticle size, concentration and location. Physics in Medicine and Biology. 2011;56(15):4631-47. doi: 10.1088/0031-9155/56/15/001. PubMed PMID: WOS:000292885000002.

15. Bao B, Groves K, Zhang J, Handy E, Kennedy P, Cuneo G, Supuran CT, Yared W, Rajopadhye M, Peterson JD. In vivo imaging and quantification of carbonic anhydrase IX expression as an endogenous biomarker of tumor hypoxia. PLoS One. 2012;7(11):e50860-e. doi: 10.1371/journal.pone.0050860. PubMed PMID: 23226406.

16. Colliez F, Gallez B, Jordan BF. Assessing tumor oxygenation for predicting outcome in radiation oncology: a review of studies correlating tumor hypoxic status and outcome in the preclinical and clinical settings. Frontiers in oncology. 2017;7:10.

17. Graham K, Unger E. Overcoming tumor hypoxia as a barrier to radiotherapy, chemotherapy and immunotherapy in cancer treatment. International journal of nanomedicine. 2018;13:6049.

18. Cooney MM, van Heeckeren W, Bhakta S, Ortiz J, Remick SC. Drug insight: vascular disrupting agents and angiogenesis--novel approaches for drug delivery. Nature clinical practice Oncology. 2006;3(12):682-92. Epub 2006/12/02. doi: 10.1038/ncponc0663. PubMed PMID: 17139319.

19. Dimcevski G, Kotopoulis S, Bjanes T, Hoem D, Schjott J, Gjertsen BT, Biermann M, Molven A, Sorbye H, McCormack E, Postema M, Gilja OH. A human clinical trial using ultrasound and microbubbles to enhance gemcitabine treatment of inoperable pancreatic cancer. Journal of controlled release : official journal of the Controlled Release Society. 2016;243:172-81. Epub 2016/11/05. doi: 10.1016/j.jconrel.2016.10.007. PubMed PMID: 27744037. 
20. Kunjachan S, Kotb S, Pola R, Pechar M, Kumar R, Singh B, Gremse F, Taleei R, Trichard F, Motto-Ros V, Sancey L, Detappe A, Yasmin-Karim S, Protti A, Shanmugam I, Ireland T, Etrych T, Sridhar S, Tillement O, Makrigiorgos G, Berbeco RI. Selective Priming of Tumor Blood Vessels by Radiation Therapy Enhances Nanodrug Delivery. Scientific Reports. 2019; 9:15844. doi: 10.1038/s41598-019-50538-w. PubMed PMID: 31676822, PMCID: PMC6825216

21. Detappe A, Kunjachan S, Drane P, Kotb S, Myronakis M, Biancur DE, Ireland T, Wagar M, Lux F, Tillement O, Berbeco R. Key clinical beam parameters for nanoparticle-mediated radiation dose amplification. Scientific Reports. 2016;6:34040. Epub 2016/09/24. doi: 10.1038/srep34040. PubMed PMID: 27658637; PMCID: PMC5034311.

22. Berbeco RI, Detappe A, Tsiamas P, Parsons D, Yewondwossen M, Robar J. Low Z target switching to increase tumor endothelial cell dose enhancement during gold nanoparticle-aided radiation therapy. Med Phys. 2016;43(1):436. Epub 2016/01/10. doi: 10.1118/1.4938410. PubMed PMID:26745936; PMCID: PMC4698122. 


\section{Clinical Translation of Gold Nanoparticles for}

\section{In-Vivo Use}

Jason R. Cook ${ }^{1}$ and J. Donald Payne ${ }^{2}$

${ }^{1}$ NanoHybrids, Inc., Austin, TX 78744, United States of America

2 Aurorad, Inc, Houston, TX 77054

\section{Status}

Currently there are 12 active or completed clinical trials involving gold nanoparticles listed on clinicaltrials.gov, as shown in Table 1. Despite a slow start, clinical translation of gold nanoparticles is accelerating toward the clinic, led by Clene Nanomedicine who is actively recruiting for Phase 2 clinical trials. Unlike many of the other technologies which utilize IV delivery, CNM-Au8 is orally administered and does not suffer from many of the off-target accumulation issues associated with systemic delivery. However, this is still a very significant milestone because it marks the first gold nanoparticle technology to move into Phase 2 effectiveness studies.

Table 1: List of active or completed clinical trials of gold nanoparticles from clinicaltrials.gov.

\begin{tabular}{|c|c|c|c|c|c|}
\hline $\begin{array}{c}\text { Clinicial } \\
\text { Trial } \\
\text { Identifier }\end{array}$ & $\begin{array}{c}\text { Year } \\
\text { Listed }\end{array}$ & & Sponsor/Company & Material & Conditions \\
\hline NCT0035698 & 2006 & Phase 1 & $\begin{array}{c}\text { NIH Clinical Cancer } \\
\text { / Cytimmune }\end{array}$ & $\begin{array}{c}\text { Aurimune: } \\
\text { TNF-target } \\
\text { conjugated and } \\
\text { PEGylated gold } \\
\text { nanosphere }\end{array}$ & $\begin{array}{c}\text { Unspecific Adult } \\
\text { Solid Tumor }\end{array}$ \\
\hline
\end{tabular}




\begin{tabular}{|c|c|c|c|c|c|}
\hline NCT00436410 & 2007 & $\begin{array}{c}\text { Early } \\
\text { Phase } 1\end{array}$ & $\begin{array}{c}\text { NIH Clinical Cancer } \\
\text { / Cytimmune }\end{array}$ & $\begin{array}{c}\text { Aurimune: } \\
\text { TNF-target } \\
\text { conjugated and } \\
\text { PEGylated gold } \\
\text { nanosphere }\end{array}$ & $\begin{array}{l}\text { Adrenocortical } \\
\text { Carcinoma, } \\
\text { Breast Cancer, } \\
\text { Colorectal Cancer, } \\
\text { Gastrointestinal } \\
\text { Cancer, } \\
\text { Kidney Cancer, } \\
\text { Liver Cancer, } \\
\text { Melanoma (Skin), } \\
\text { Ovarian Cancer, } \\
\text { Pancreatic Cancer, } \\
\text { and } \\
\text { Sarcoma }\end{array}$ \\
\hline NCT00848042 & 2009 & $\begin{array}{c}\text { Not } \\
\text { Applicable }\end{array}$ & $\begin{array}{l}\text { Nano } \\
\text { Bios }\end{array}$ & $\begin{array}{c}\text { AuroLase: PEG } \\
\text { coated gold } \\
\text { nanoshell }\end{array}$ & $\begin{array}{c}\text { Head and Neck } \\
\text { Cancer }\end{array}$ \\
\hline NCT01270139 & 2011 & $\begin{array}{r}\text { Not } \\
\text { Applica }\end{array}$ & $\begin{array}{l}\text { Ural Medical } \\
\text { University }\end{array}$ & $\begin{array}{c}\text { Silica-gold } \\
\text { nanoparticle }\end{array}$ & Cardiovascular \\
\hline NCT02680535 & 2016 & & $\begin{array}{l}\text { Nanospectra } \\
\text { Biosciences }\end{array}$ & $\begin{array}{c}\text { AuroLase: PEG } \\
\text { coated gold } \\
\text { nanoshell }\end{array}$ & $\begin{array}{c}\text { Neoplams of the } \\
\text { Prostate }\end{array}$ \\
\hline NCT02837094 & 2016 & Phase 1 & $\begin{array}{l}\text { Cardiff University / } \\
\text { Midatech Pharma }\end{array}$ & $\begin{array}{c}\text { MidaCore } \\
\text { MTX102: } \\
\sim 1.5 \mathrm{~nm} \\
\text { diameter Gold } \\
\text { nanoparticle } \\
\text { coated with }\end{array}$ & Type 1 Diabetes \\
\hline
\end{tabular}




\begin{tabular}{|c|c|c|c|c|c|}
\hline & & & & $\begin{array}{l}\text { C19-A3 } \\
\text { peptides, L- } \\
\text { glutathione, } \\
\text { and glucose }\end{array}$ & \\
\hline NCT02755870 & 2016 & Phase 1 & $\begin{array}{c}\text { Clene } \\
\text { Nanomedicine }\end{array}$ & $\begin{array}{l}\text { CNM-Au8: } \\
\text { 10nm } \\
\text { diameter Gold } \\
\text { Nanocrystal }\end{array}$ & $\begin{array}{l}\text { Healthy } \\
\text { Volunteers - Male } \\
\text { and Female }\end{array}$ \\
\hline NCT03020017 & 2017 & $\begin{array}{c}\text { Early } \\
\text { Phase } 1\end{array}$ & 1 & NU-0129 & $\begin{array}{c}\text { Gliosarcoma } \\
\text { Recurrent } \\
\text { Glioblastoma }\end{array}$ \\
\hline NCT03669224 & 2018 & & Cairo University & $\begin{array}{c}\text { Nano Care } \\
\text { Gold: Gold and } \\
\text { silver } \\
\text { nanoparticles } \\
\text { suspended in } \\
70 \% \text { isopropyl } \\
\text { alcohol }\end{array}$ & Dentistry \\
\hline NCT03536559| & 2018 & Phase 2 & $\begin{array}{c}\text { Clene } \\
\text { Nanomedicine }\end{array}$ & $\begin{array}{l}\text { CNM-Au8: } \\
\text { 10nm } \\
\text { diameter Gold } \\
\text { Nanocrystal }\end{array}$ & $\begin{array}{c}\text { Relapsing } \\
\text { Remitting } \\
\text { Multiple Sclerosis, } \\
\text { Optic Neuropathy, } \\
\text { and }\end{array}$ \\
\hline
\end{tabular}




\section{Current and future challenges}

Despite more than 50,000 articles listed on Google Scholar with the keywords gold nanoparticles and in vivo, no gold nanoparticle-based intervention has received FDA approval for clinical use. Many of these technologies could have significant clinical merit, but that alone is not enough to garner clinical translation. Most of the work with gold nanoparticles has been performed in academic laboratories, and in order to achieve some sort of publication the investigators must demonstrate some sort of feasibility that their gold nanoparticle-based technology can work for a particular application. Although many of these publications are significant contributions, most of the work is devoted to scientific discovery and not clinical translation. Unfortunately, this leaves a gap between academic development and clinical product development. Once feasibility in a technology has been established, financial funding is the main determinant of clinical translation. As shown in Figure 1, public grants can only take a technology so far, and eventually will need to rely on private funding. 
The finances involved with any clinical product development are very substantial and generally investors weigh the finances with how long to receive FDA approval and return on investment. Gold nanoparticle technologies developed based on scientific discovery generally have a lot of innovative aspects to them, which equals additional regulatory oversight and a longer path to clinic. Both public (e.g., business grants) and private investors are generally less enthusiastic about these type of technologies as they can often be considered too high risk investments.

Assuming that the technology has the correct mix of innovation while being simple enough to have a relatively quick path to the clinic, there are a variety of different funding sources, as shown in Figure 1. Because many technologies start in academic laboratories, the initial funding source is government grants. A technology can be backed by public funding for a 
significant portion of the clinical product development. There is precedent that public funding has taken technologies as far as early clinical trials, however most technologies must make the jump from public to private funding sometime before Good Manufacturing Practices (GMP) production is required.

Gold nanoparticles are not trivial to manufacture, requiring high purity ingredients and reactors. Further complicating the manufacture is that gold nanoparticle formation is a stochastic process and requires extremely expensive characterization equipment (e.g. transmission electron microscopy). If a biomolecular targeting group is involved (e.g. antibody, peptide, nucleic acid, affibody, or anything that could cause an immunologic response) then the required quality controls (QCs) and quality assurances (QAs) are even more complex. Once the quality systems are established and the gold nanoparticle manufacture is fully integrated into current GMP, then the next step will be extensive toxicity studies. To be cleared by the FDA to perform a clinical trial, these toxicity studies must be performed under Good Laboratory Practices (GLP). Maintaining a GLP facility is extremely expensive and therefore most technologies outsource these studies to a GLP-certified contract research organization (CRO). The International Council for Harmonisation of Technical Requirements for Pharmaceuticals for Human Use (ICH) has outlined most of the guidelines for toxicity studies, and depending on the method of action of the gold nanoparticle, will determine the number of animal species and testing burden required. For simple gold nanoparticle interventions, ones with no biologic and only a single low dose, the GLP toxicity study costs can be less than $\$ 100,000$. However, complex interventions that include a biologic, or a known toxic agent, and multiple dosing could escalate costs to over $\$ 10,000,000$.

Investments of this level are not readily available for a technology class that has not been approved by the FDA. Therefore, many of the technologies entering clinical trials are generally considered as 'high-risk high-reward'. Due to the nature of these technologies, clinical testing will likely proceed for quite some time until approval can be obtained. Once one gold nanoparticle-based technology is approved, then some of the required GLP toxicity studies and clinical trials may be bypassed. This will greatly reduce commercialization times and costs for 
subsequent products seeking FDA clearance, encouraging more private investments and driving more gold nanoparticle-based technologies to the clinic.

\section{Advances in science and technology to meet challenges}

The major concerns with the FDA is safety and effectiveness, and until recently none of the gold nanoparticle technologies have moved past Phase 1 in-human safety studies. Although this may not necessarily be the case of these technologies, some gold nanoparticles are known to suffer from poor clearance from the body.[2] It has been shown that gold largely accumulates in the mononuclear phagocyte system (MPS) (also known as the reticuloendothelial system) because the body cannot break-down gold and most technologies require gold nanoparticles too large to be cleared by the kidneys. Therefore, if the technology uses gold as a drug delivery vehicle, there are questions as to why gold should be chosen as opposed to other existing technologies. If the technology uses gold as an energy converter for therapy (e.g., photothermal therapy or radiation therapy) or imaging contrast (e.g., photoacoustic/optoacoustic imaging), then questions about dosage and biodistribution are at the forefront. Therefore, current systemically delivery applications of gold nanoparticles are limited to high-morbidity indications.

Advances in delivery and clearance will probably have the most profound influence on the future of clinical translation of gold nanoparticles. Effective delivery, either by improving bioavailability for lower effective doses or molecular targeting strategies, could have a profound effect on the potential safety profile of gold nanoparticles. If the clearance problem is solved, then long-term accumulation concerns will be reduced and could open gold nanoparticle applications for low-morbidity interventions.

\section{Concluding remarks}

The clinical presence of gold nanoparticle is on the rise, with at least eight new clinical trials listed in the past three years. Despite the financial burden to get a technology through feasibility and preclinical testing just to get a shot of testing into humans, several companies/groups have demonstrated that it can happen and is happening at an increasing 
rate. The presence of the Phase 2 trials is really encouraging for the gold nanoparticle field, since it signifies the first time that gold nanoparticles have moved past Phase 1 safety studies. Hopefully these pioneering clinical trials pave the way for many more gold nanoparticle-based technologies, ushering in entirely new classes of diagnostics and therapeutics.

\section{References:}

[1] Steinmetz, K. L., \& Spack, E. G. (2009). The basics of preclinical drug development for neurodegenerative disease indications. BMC neurology, 9 Suppl 1(Suppl 1), S2. doi:10.1186/1471-2377-9-S1-S2

[2] Hoshyar, N., Gray, S., Han, H., \& Bao, G. (2016). The effect of nanoparticle size on in vivo pharmacokinetics and cellular interaction. Nanomedicine (London, England), 11(6), 673-692. doi:10.2217/nnm.16.5 


\title{
Next-Generation Metallic Nanoparticles for
}

\section{Cancer Radiation Therapy}

\author{
Bijay Singh, Srinivas Sridhar \\ Department of Physics, Northeastern University, Boston MA 02115
}

\section{Status}

High Z elements (heavy metals) behave as potent radiosensitizers because they can produce a cascade of Auger electrons when ionized by X-ray irradiation [1]. In fact, the increment of resonant energies in heavy metals after irradiation is adequately high to ensure significant penetration in body tissue [2]. Laboratory experiments, using heavy metals injected into tumors and then irradiated with high-energy X-rays, have exhibited considerable reduction in tumor sizes [3]. Most importantly, the in situ deposition of radiation energy, followed by secondary photon and electron emission, will be confined at the irradiated tumor site. Due to these benefits, various formulations made of heavy metals have been conceived and developed to improve the radiation dose enhancement for cancer treatments.

\section{Current and future challenges}

Among high $Z$ elements, gold $(Z=79)$ has been the prime choice for radiation therapy due to its biocompatibility and low toxicity. Although the earliest studies with bulk or larger particles of gold to enhance radiation dose were successful in vitro, the larger 
gold particles failed to show their effectiveness in vivo. Alternatively, gold nanoparticles (AuNPs) with diameter (1-5 nm) were produced and intravenously injected to enhance the accumulation of these ultrasmall particles in tumor tissues. As a consequence, when the tumor was irradiated, the 1 -year survival was $86 \%$ compared to $20 \%$ with radiation alone and $0 \%$ with gold alone [3]. Following the successful result, innumerable studies have focused on the optimization of composition and size of AuNPs, uptake of nanoparticles into cells, and the dose of applied radiation.

A major problem of AuNPs is their short circulation in the blood stream after injection, requiring immediate irradiation. But, a high dose of AuNPs, required for satisfactory treatment of tumors, is impractical for human use, partly due to toxicity concerns. To use the AuNPs for clinical applications, the size, surface chemistry and targeting ability of AuNPs could be tuned for longer circulation time and enhanced accumulation in specific tumors. A vast array of chemical agents (cysteamine, glucose, choline, citrate, folic acid), biomolecules (nucleotides, peptides, antibodies) and polymers (synthetic, natural) are available to conjugate to AuNPs to facilitate the higher uptake of particles in tumors. Although targeting agents may ameliorate the delivery of AuNPs to tumor sites, it is not fully understood whether these modified particles really assist radiation for tumor therapy. Again, optimization of AuNPs with optimum density of targeting agents and delivery routes (intravenous, intratumoral) is necessary prior to clinical translation. To date, only a few clinical trials $(<10)$ of AuNPs are currently underway for cancer treatment [4] [5] [6].

\section{Advances in science and technology to meet challenges}


In pursuit of alternatives to AuNPs, other high $Z$ elements such as bismuth $(Z=83)$, platinum $(Z=78)$ and gadolinium $(Z=64)$ are currently being investigated to prepare the formulations for radiation therapy. Under similar conditions of size and concentration of particles, bismuth provides higher dose enhancements than gold while platinum shows equivalent efficacy to gold [7]. Particularly, bismuth oxide nanoparticles (BiNPs) are verified as efficient dose enhancing agents demonstrating their potential application in clinical radiotherapy [8]. Bismuth selenide nanoplates have shown significant radiation dose enhancement both in vitro and in vivo [9]. Of note, these nanoplates were selectively accumulated in tumor tissue after intraperitoneal injection into mice. The higher concentration of nanoplates not only enhanced the contrast of X-ray computerized tomography (CT) images but also led to damage on the tumor cells when exposed to radiation.

A targeted radiotherapeutic agent was developed from bismuth nanosheets coated with chitosan and RGD peptide [10]. The nanosheets exhibit targeting ability to av $\beta 3$ integrin-overexpressing HeLa cells with higher radiosensitization efficiency. When the mice bearing HeLa tumors were irradiated after treatment with the nanosheets by intravenous or intratumoral injection, the tumor volume decreased significantly. Importantly, the nanosheets can be used for photoacoustic imaging and magnetic resonance (MR) imaging to study their targeting ability and therapeutic effects together. Thus, this study reveals an effective theranostic agent for next-generation cancer radiotherapy. In another study, functionalization of BiNPs with hyaluronic acid enhanced water solubility, biocompatibility and targeting ability to cancer cells 
overexpressing CD44 receptors [11]. These particles offer great promise for targetspecific CT imaging and radiosensitization of tumors.

Although nanoparticles of high $Z$ elements are used as radiosensitizers to enhance radiation therapy, the non-degradable nature of the nanoparticles, that can cause longterm toxicity when accumulated inside body, is a major bottleneck in translating nanoparticle radiosensitizers into clinical application for cancer treatment. To address the issue, a new formulation of folate and red blood cell membrane (F-RBC) combined BiNPs was developed, where folate targets tumor and cell membrane coating provides longer blood circulation time [12]. When F-RBC BiNPs were used to sensitize radiation for breast cancer treatment, there was significant tumor inhibition in mice resulting in improved survival ratios. Histological and in vivo bio-distribution analyzes revealed FRBC BiNPs were excreted after 15 days from the animal body with no evident damage or inflammatory response in major organs.

Gadolinium ( $Z=64)$ is another alternative to gold which has been routinely used as a contrast agent in MR imaging. For instance, gadolinium oxide nanoparticles behave as efficient positive contrast agents for MR imaging and show radiosensitizing effects in gliosarcoma rat models [13]. In a new report, bovine serum albumin coated gadolinium nanoclusters were developed as multifunctional theranostics that could be used for CT or MR imaging and photothermal or radiation therapy of tumor treatment [14]. Moreover, the low toxicity and efficient renal clearance of these theranostics suggest their practical applications for cancer diagnoses and therapies. A new gadolinium nanoparticle agent, named AGuIX (Activation and Guidance of Irradiation by X-Ray), has recently been established as a potent radiosensitizer in cancer treatment. AGuIX 
has high capacity of absorption of photons delivered by a radiotherapy beam which generates an enhanced local dose deposit. Accordingly, combining AGulX with radiation therapy greatly enhanced tumor cell death, and improved the survival ratio of animals with multiple brain melanoma metastases [15]. Currently, the first phase I trial is going on to study the side effects and optimum dose of AGuIX when given together with whole brain radiation therapy in treating patients with multiple brain metastases [16]. Magnetic particles are another option to use in cancer radiation therapy in combination with hyperthermia. Hyperthermia is a treatment of cancer by exposing tumor tissues to high temperatures which can damage and kill cancer cells, usually with minimal harm to normal tissues. Hyperthermia may induce some cancer cells to be more sensitive to radiation or damage the cancer cells that radiation cannot kill. Iron oxide nanoparticles (FeNPs), due to their low toxicity and ability to emit heat energy when excited by magnetic fields, are generally used in hyperthermia. Additionally, FeNPs are used as contrast agents for MR imaging. Due to these benefits, several varieties of FeNPs have been produced for cancer therapy. Typically, dextran-coated FeNPs have shown to decrease tumor growth in a breast cancer model using hyperthermia and irradiation [17]. Most importantly, both intracranial and intratumoral hyperthermia using FeNPs combined with radiotherapy could be safely applied on patients with glioblastoma multiforme [18] [19]. A few studies have also focused on the radiosensitization properties of FeNPs. Irradiation on human prostate carcinoma cell line (DU145) with FeNPs $(1 \mathrm{mg} / \mathrm{ml})$ produced a dose enhancement factor of approximately 1.2 [20]. In another study, several tumor cells were irradiated in the presence of citrate- or malatecoated FeNPs, where a drastically increased concentration of reactive oxygen species 
in the tumor cells verified these nanoparticles as potent radiosensitizers for radiation cancer therapy [21].

Recently, lodine nanoparticles (INPs) are developed for radiotherapy to overcome the drawbacks of AuNPs in clinical translation [22]. The major drawbacks of AuNPs are due to cost, skin discoloration and poor body clearance. In contrast, INPs have unique characteristics; they are almost colorless, non-toxic, and cost-effective in manufacturing. Above all, INPs show higher body clearance from the liver (50\% in 6 months) compared to AuNPs (9\% in 6 months) [23]. Experimentally, INPs when combined with radiotherapy or chemotherapy extended the survival period of the treated groups [22]. In addition, INPs have compatible size $(20 \mathrm{~nm})$ for better tumor penetration compared to liposomes (>100 nm) [24], and they, being coated with PEG, have longer blood half-life (40 hours) for better tumor uptake. When injected through intravenous route, INPs strongly accumulates in tumors at high levels providing extended-time for high contrast vascular and tumor imaging [26].

\section{Concluding remarks}

Although significant progress has been made in radiation therapy for cancer treatment using metallic nanoparticles, the inherent radioresistance and inaccuracy of localization of nanoparticles in tumors weaken the clinical implementation. Hence, the development of nanosized particles with versatile properties (biocompatibility, low toxicity and targeting efficaly) to use in imaging-guided radiation therapy has always been a top priority in biomedical research. Next generation metallic nanoparticles decorated with 
biocompatible coating and functionalized with ligands specific to cancer cells will solve the current problems of imaging and therapy.

\section{References}

1. Kobayashi, K., et al., Enhancement of radiation effect by heavy elements. Mutat Res, 2010. 704(1-3): p. 123-31.

2. Pradhan, A.K., et al., Resonant X-ray enhancement of the Auger effect in high-Z atoms, molecules, and nanoparticles: potential biomedical applications. J Phys Chem A, 2009. 113(45): p. 12356-63.

3. Hainfeld, J.F., D.N. Slatkin, and H.M. Smilowitz, The use of gold nanoparticles to enhance radiotherapy in mice. Phys Med Biol, 2004. 49(18): p. N309-15.

4. Libutti, S.K., et al., Phase I and pharmacokinetic studies of CYT-6091, a novel PEGylated colloidal gold-rhTNF nanomedicine. Clin Cancer Res, 2010. 16(24): p. 6139-49.

5. Bobo, D., et al., Nanoparticle-Based Medicines: A Review of FDA-Approved Materials and Clinical Trials to Date. Pharmaceutical research, 2016. 33(10): p. 2373-2387.

6. Singh, P., et al., Gold Nanoparticles in Diagnostics and Therapeutics for Human Cancer. International journal of molecular sciences, 2018. 19(7): p. 1979.

7. Hossain, M. and M. Su, Nanoparticle location and material dependent dose enhancement in Xray radiation therapy. J Phys Chem C Nanomater Interfaces, 2012. 116(43): p. 23047-23052.

8. Alqathami, M., et al., Enhancement of radiation effects by bismuth oxide nanoparticles for kilovoltage $x$-ray beams: A dosimetric study using a novel multi-compartment 3D radiochromic dosimeter. Journal of Physics: Conference Series, 2013. 444: p. 012025.

9. Zhang, X.-D., et al., Metabolizable Bi2Se3 Nanoplates: Biodistribution, Toxicity, and Uses for Cancer Radiation Therapy and Imaging. 2014. 24(12): p. 1718-1729.

10. Song, Z., et al., Decorated ultrathin bismuth selenide nanosheets as targeted theranostic agents for in vivo imaging guided cancer radiation therapy. Npg Asia Materials, 2017. 9: p. e439.

11. Du, F., et al., Hyaluronic acid-functionalized bismuth oxide nanoparticles for computed tomography imaging-guided radiotherapy of tumor. Int J Nanomedicine, 2017. 12: p. 5973-5992.

12. Deng, J., et al., Tumor targeted, stealthy and degradable bismuth nanoparticles for enhanced Xray radiation therapy of breast cancer. Biomaterials, 2018. 154: p. 24-33.

13. Le Duc, G., et al., Toward an image-guided microbeam radiation therapy using gadolinium-based nanoparticles. ACS Nano, 2011. 5(12): p. 9566-74.

14. Yong, Y., et al., Gadolinium polytungstate nanoclusters: a new theranostic with ultrasmall size and versatile properties for dual-modal MR/CT imaging and photothermal therapy/radiotherapy of cancer. Npg Asia Matérials, 2016. 8: p. e273.

15. Kotb, S., et al., Gadolinium-Based Nanoparticles and Radiation Therapy for Multiple Brain Melanoma Metastases: Proof of Concept before Phase I Trial. Theranostics, 2016. 6(3): p. 418427.

16. Verry, C., et al., Treatment of multiple brain metastases using gadolinium nanoparticles and radiotherapy: NANO-RAD, a phase I study protocol. BMJ Open, 2019. 9(2): p. e023591.

17. Giustini, A.J., A.A. Petryk, and P.J. Hoopes, Comparison of microwave and magnetic nanoparticle hyperthermia radiosensitization in murine breast tumors. Proc SPIE Int Soc Opt Eng, 2011. 7901. 
18. Maier-Hauff, K., et al., Intracranial thermotherapy using magnetic nanoparticles combined with external beam radiotherapy: results of a feasibility study on patients with glioblastoma multiforme. J Neurooncol, 2007. 81(1): p. 53-60.

19. Maier-Hauff, K., et al., Efficacy and safety of intratumoral thermotherapy using magnetic ironoxide nanoparticles combined with external beam radiotherapy on patients with recurrent glioblastoma multiforme. J Neurooncol, 2011. 103(2): p. 317-24.

20. Khoei, S., et al., The role of iron oxide nanoparticles in the radiosensitization of human prostate carcinoma cell line DU145 at megavoltage radiation energies. Int J Radiat Biol, 2014. 90(5): p. 351-6.

21. Klein, S., et al., Superparamagnetic iron oxide nanoparticles as novel X-ray enhancer for low-dose radiation therapy. J Phys Chem B, 2014. 118(23): p. 6159-66.

22. Hainfeld, J.F., et al., lodine nanoparticles enhance radiotherapy of intracerebral human glioma in mice and increase efficacy of chemotherapy. Scientific reports, 2019. 9(1): p. 4505-4505.

23. Sadauskas, E., et al., Protracted elimination of gold nanoparticles from mouse liver. Nanomedicine : nanotechnology, biology, and medicine, 2009. 5(2): p. 162-169.

24. Perrault, S.D., et al., Mediating tumor targeting efficiency of nanoparticles through design. Nano letters, 2009. 9(5): p. 1909-1915.

25. Hainfeld, J.F., et al., Small, Long Blood Half-Life lodine Nanoparticle for Vascular and Tumor Imaging. Scientific reports, 2018. 8(1): p. 13803-13803. 


\section{Theranostics Metallic Nanoparticles}

Udoka M. Ibeh, Wilfred Ngwa

Brigham and Woman's Hospital \& Harvard Medical School, Boston, MA

\section{Status}

In recent years, the field of nanomedicine has experienced significant growthin research interest. The use of metallic nanoparticles (MNPs) has been especially notable due to both the number of scientific publications advancing the field and the scope of applications.

Advancements in synthesis, conjugation, transport and distribution patterns of nanoparticles have allowed for their use in numerous biomedical applications. Coupling the diagnostic and therapeutic capabilities of these MNPs to form theranostic agents is an active area of research. The therapeutics research area seeks to increase nanoparticle targeting, treatment dose distribution, and multidrug delivery. Concurrently, the diagnostics area provides improved image quality and enhanced contrasts. Nanoparticle theranostics harnesses the multi-functionality of MNPs to allow for continuous diagnosis or assessment of patients before, during and after therapy to better understand and increase treatment efficacy. This would allow for the development of more tailored therapies, and improvements in patient prognosis ${ }^{1,2}$.

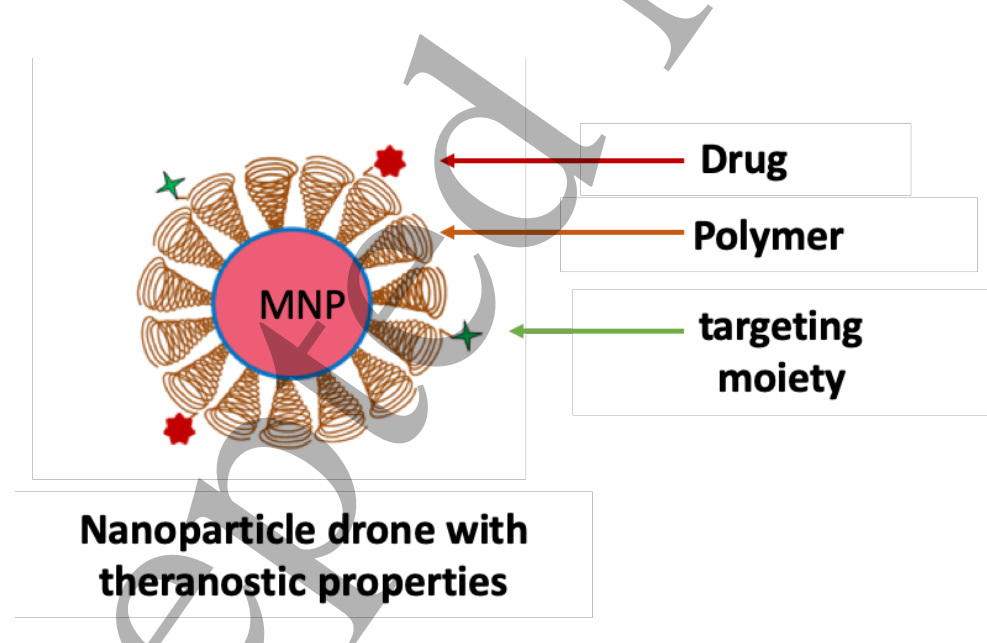

Figure 1: Metallic Nanoparticle designed for theranostic applications.

The synthesis of nanoparticle theranostic agents can occur in several ways. Therapeutic nanoparticles can be conjugated with contrast agents such as radioisotopes or additional imaging contrast agents to induce additional diagnostic imaging functionalities. Conversely, imaging 
nanoparticles can be conjugated with therapeutic agents such as drugs, radio and photosensitizers, and therapeutic moieties to induce therapeutic functionalities ${ }^{3}$. Certain metallic nanoparticles have intrinsic theranostic (therapeutic and imaging contrast) capability (e.g. gadolinium or gold nanoparticles). Other nanoparticles may be hybrid nanoparticlescomposed of more than one metal. Such combination promises to be more effective as it can make use of the inherent positive characteristics of each metal making up its composition ${ }^{4}$. Much focus has been placed on refining nanoparticle synthesis as it is an important determinant of its functionality.

Due to the inherently complex nature of biological systems, nanoparticles must be optimized to have specific and measurable mechanics. They must have large enough blood circulation half-lives to allow them to reach their targeting site. In their therapeutic applications, they must be able to efficiently deliver a specific dose of drugs to a localized region so as to decrease toxicities. Once delivered, they must be able to be rapidly and specifically accumulate within biological targets of interest in order to reduce collateral damage and to increase treatment specificity. In their diagnostic applications, they must be able to analyze and report the biological, biochemical and morphological characteristics of the tissue or disease of interest in an effective way. After performing their theranostic tasks, they must be effectively degraded into noncytotoxic byproducts or be cleared from the body quickly and effectively without inducing any toxicities ${ }^{3}$.

\section{Current and Future Challenges}

Most nanoparticles when used in solid tumors for imaging and drug delivery rely on the Enhanced Permeability and Retention (EPR) effect to localize within the tumor microenvironment. This EPR effect is induced by aberrant tumor vasculatures that are characteristic of most solid tumors ${ }^{5-7}$. Unfortunately, it also leads to extensive difficulties when attempting to determine how to best optimize nanoparticles for specific targeting - as it becomes significantly difficult to determine environmental conditions and create predictable diffusion or transport mechanisms ${ }^{5,8}$. Engineering nanoparticles that have dynamic active targeting capabilities is an important field of interest.

A significant hurdle in the use of nanoparticle theranostics in clinical settings is due to an inability to generate theranostic agents capable of meeting all the above-mentioned nanoparticle 
optimization criteria. In the current state of nanoparticle synthesis, strides in one direction often require that compromise be made in other directions. For example, increasing blood circulation half-life often leads to increased toxicity. Conversely, conjugating nanoparticles with tumorspecific ligands for increased binding often leads to decreased blood circulation half-life. Discerning methods to simultaneously achieve these criteria without sacrificing any is an active area of research ${ }^{1,3}$

Several parameters that must be optimized for the use of these nanoparticles in everyday clinical practice includes: optimizing nanoparticle materials based on their intended goals, increasing the efficiency of ligand conjugations, decreasing the cost of nanoparticle synthesis and conjugation, and development of methods that allow for consistent reproducible nanoparticle synthesis such that their size, density, and material composition remains homogenous. Furthermore, nanoparticles must be designed to be able to avoid innate immune recognition, though they could also be tailored to enhance immunotherapy.

\section{Advances in science and technology to meet challenges}

Metallic nanoparticles have intrinsic physicochemical and biocompatible properties that make them strong candidates for theranostic applications. Gold nanoparticles have been shown to be relatively noncytotoxic, to increase the therapeutic ratio of radiotherapy, and to improve local imaging ${ }^{9-13}$. Recent advancements in this space have led to the development of metallic nanoparticle conjugates capable of selectively binding tumor-specific ligands. One such example utilizes the angiogenic markers characteristic of many tumors. A second mechanism is the generation of folate-conjugated porphysomes based nanoparticles which utilize the fact that the folate receptor, a glycosylphosphatidylinositol-linked membrane protein, is highly expressed in many tumors and has a high affinity for folic acid ${ }^{14-17}$.

Because nanoparticle theranostics is a combination of the diagnostic and therapeutic fields, independent progress in any of these two fields, theoretically can advance theranostics; one type can simply be conjugated with the characteristics of the other to form theranostic capabilities $^{6}$. The Food and Drug Administration (FDA) has successfully accepted more than 35 nanoparticles for therapeutic and image diagnostic use in clinical trials ${ }^{2,3}$. These nanoparticles can be employed as platforms to incorporate theranostic agents and thus be able to reach clinical trials which will allow for expedited approval for clinical trials. 
Substantial effort has gone toward formulating mechanisms that increase the circulatory half-life of theranostic nanoparticles. By coating nanoparticle theranostics with cellular membrane of leukocytes and red blood cells, their susceptibility to immune recognition is considerably minimized ${ }^{18}$. These nanoparticles can also be synthesized to show active targeting of select tumors by conjugating multiple targeting ligands to the nanoparticle. Furthermore, one active area of research looks to map out the diffusion and accumulation of varying nanoparticle sizes on breaking through the vasculature epithelium to accumulate in tumor microenvironments ${ }^{8}$.

\section{Conclusion}

Nanoparticle Theranostics using MNPs has become a very active field of research and promises to both enhance therapeutic delivery and retention of chemotherapeutics, photosensitizing and radiosensitizing agents, as well as improving imaging quality. Although only few nanoparticle platforms have reached clinical trials, the significant amount of interest and research activity in this space promises to quickly change that. The use of nanoparticles as theranostic agents will help enhance personalized disease therapy by enabling continuous and concomitant diagnosis and treatment. Developing agents capable of long blood circulation, specific therapeutic dose delivery, decreased toxicity and an ability to specifically accumulate within a target region are all avenues of ongoing studies.

\section{References}

1. Xie J, Lee S, Chen X. Nanoparticle-based theranostic agents. Adv Drug Deliv Rev. 2010;62(11):1064-1079. doi:10.1016/j.addr.2010.07.009

2. Thakor AS, Gambhir SS. Nanooncology: The future of cancer diagnosis and therapy. $C A$ Cancer J Clin. 2013;63(6):395-418. doi:10.3322/caac.21199 
3. Chen F, Ehlerding EB, Cai W. Theranostic Nanoparticles. doi:10.2967/jnumed.114.146019

4. Hoskins C, Min Y, Gueorguieva M, et al. Hybrid gold-iron oxide nanoparticles as a multifunctional platform for biomedical application. J Nanobiotechnology. 2012;10(1):27. doi:10.1186/1477-3155-10-27

5. Chen F, Cai W. Tumor Vasculature Targeting: A Generally Applicable Approach for Functionalized Nanomaterials. 2014. doi:10.1002/smll.201303627

6. Xie J, Lee S, Chen X. Nanoparticle-based theranostic agents. doi:10.1016/j.addr.2010.07.009

7. Fang J, Nakamura H, Maeda H. The EPR effect: Unique features of tumor blood vessels for drug delivery, factors involved, and limitations and augmentation of the effect. $A d v$ Drug Deliv Rev. 2011;63(3):136-151. doi:10.1016/j.addr.2010.04.009

8. Smith BR, Kempen P, Bouley D, et al. Shape Matters: Intravital Microscopy Reveals Surprising Geometrical Dependence for Nanoparticles in Tumor Models of Extravasation. doi:10.1021/n1204175t

9. Pietro P Di, Strano G, Zuccarello L, Satriano C. Gold and Silver Nanoparticles for Applications in Theranostics. Curr Top Med Chem. 2016;16(27):3069-3102. http://www.ncbi.nlm.nih.gov/pubmed/27426869. Accessed May 2, 2019.

10. Hainfeld JF, Slatkin DN, Focella TM, Smilowitz HM. Gold nanoparticles: a new X-ray contrast agent. Br J Radiol. 2006;79(939):248-253. doi:10.1259/bjr/13169882

11. Ngwa W, Korideck H, Kassis AI, et al. In vitro radiosensitization by gold nanoparticles during continuous low dose rate gamma irradiation with I-125 brachytherapy seeds. 2012. doi:10.1016/j.nano.2012.09.001

12. Ngwa W, Kumar R, Sridhar S, et al. Targeted radiotherapy with gold nanoparticles: Current status and future perspectives. Nanomedicine. 2014;9(7):1063-1082. doi: $10.2217 / \mathrm{nnm} .14 .55$

13. Hainfeld JF, Slatkin DN, Smilowitz HM. The use of gold nanoparticles to enhance radiotherapy in mice. Phys Med Biol. 2004;49(18):N309-N315. doi:10.1088/00319155/49/18/N03

14. Sudimack J, Lee RJ. Targeted drug delivery via the folate receptor. Adv Drug Deliv Rev. 2000;41(2):147-162. http://www.ncbi.nlm.nih.gov/pubmed/10699311. Accessed May 16, 
2019.

15. Jin CS, Cui L, Wang F, Chen J, Zheng G. Targeting-Triggered Porphysome Nanostructure Disruption for Activatable Photodynamic Therapy. Adv Healthc Mater. 2014;3(8):12401249. doi:10.1002/adhm.201300651

16. Yue C, Liu P, Zheng M, et al. IR-780 dye loaded tumor targeting theranostic nanoparticles for NIR imaging and photothermal therapy. Biomaterials. 2013;34(28):6853-6861. doi:10.1016/j.biomaterials.2013.05.071

17. Santra S, Kaittanis MC, Grimm J, Manuel Perez J. Drug/Dye-Loaded, Multifunctional Iron Oxide Nanoparticles for Combined Targeted Cancer Therapy and Dual Optical/MRImaging ** NIH Public Access. Small. 2009;5(16):1862-1868.

doi:10.1002/smll.200900389

18. Piao J-G, Wang L, Gao F, You Y-Z, Xiong Y, Yang L. Erythrocyte Membrane Is an Alternative Coating to Polyethylene Glycol for Prolonging the Circulation Lifetime of Gold Nanocages for Photothermal Therapy. ACS Nano. 2014;8(10):10414-10425. doi:10.1021/nn503779d 


\title{
Metallic Nanoparticles for Enhancing
}

\section{Radiotherapy and Immunotherapy}

\author{
Alexander F. Bagley ${ }^{1}$, Wassana Yantasee ${ }^{2}$, Sunil Krishnan ${ }^{3}$ \\ 1 Department of Radiation Oncology, The University of Texas MD Anderson Cancer Center, Houston, TX \\ 2 Department of Biomedical Engineering, Oregon Health \& Science University, Portland, OR \\ ${ }^{3}$ Department of Radiation Oncology, Mayo Clinic Florida, Jacksonville, FL
}

\section{Status}

The treatment of cancer has been transformed by immunotherapy, which now represents a fourth pillar of modern cancer therapy with surgery, radiotherapy, and chemotherapy. For many cancers, immunotherapy is combined with standard therapies to improve locoregional tumor control and overall survival rates. However, many patients receiving immunotherapy fail to achieve long-term, durable responses. A recent clinical trial for head and neck cancer showed that $20 \%$ of patients achieved a radiographic treatment response to immunotherapy, while responses in melanoma, renal cell carcinoma, and lung cancer range from 20-25\%.[1,2] Innovative strategies are needed to bolster immunotherapy responses.

In certain clinical contexts, radiotherapy can act synergistically with immunotherapy. Radiotherapy triggers immunological cell death (ICD), release of tumor-associated antigens (TAAs), and radiation-induced neoantigens. Modulation of the tumor microenvironment (TME) following radiotherapy elicits immunostimulatory effects such as cytotoxic T-cell infiltration. While immunosuppressive factors in the TME offset some of the salutary effects of radiation, coupling radiotherapy with immunotherapy can tip the scale towards more immunogenic effects.[3] Recent data from patients treated with radiotherapy combined with immunotherapy suggest that this approach may have clinical utility.[4-6]

An area of interest for radiotherapy and immunotherapy synergy is the abscopal effect. Proponents posit that the local effects of radiotherapy can activate systemic anti-tumor immunity and cause regression of distant, non-irradiated tumors. While the abscopal 
effect has been reported in preclinical models and case reports, approaches to reproducibly elicit abscopal responses in patients remain undefined.

Given the role of metallic nanoparticles (MNPs) as radiotherapy enhancers, there is now interest in determining whether MNPs may likewise enhance immunotherapy. The delivery of immunostimulatory agents into the TME may also be facilitated by nanoparticle carriers. Through their dual roles as radiotherapy enhancers and carriers of immunostimulatory agents, nanomedicines could have an increasing role in the next generation of immunotherapy-based treatments.

\section{Current and Future Challenges}

Initial case reports of the abscopal effect generated significant interest and have led to hundreds of preclinical studies and clinical trials combining radiotherapy and immunotherapy.[7] However, it remains uncertain how to reproducibly elicit abscopal effects in patients. For nanomedicine to impact immuno-oncology, at least three questions must be addressed: (1) Can nanomaterials amplify radiotherapy-induced ICD and release of TAAs? (2) Can nanomaterials modulate the immunosuppressive TME? (3) Can nanomaterials-based treatment strategies limit immune-related systemic toxicities?

Preclinical data suggest that the radiation dose per fraction is correlated with activation of anti-tumor immunity Doses greater than 8 Gy per fraction increased anti-tumor immunity via the cGAS/STING pathway as measured by interferon-beta (IFN-b) expression, activated dendritic cells (DCs), and infiltrating cytotoxic T-cells. Doses above 20 Gy per fraction diminished anti-tumor immunity via activation of the DNAse Trex1, suggesting an optimal window in which radiotherapy bolsters anti-tumor immunity.[8] A recent study of ablative radiotherapy combined with CTLA-4 immune checkpoint inhibitors reported increased T-cell diversity and cytotoxic T-cell expansion and decreased immunosuppressive regulatory T-cells.[9] MNPs including gold and hafnium oxide nanoparticles are well-established as radiosensitizers via the increased 
production of secondary electrons.[10] Emerging data suggest that MNPs may lead to increased anti-tumor immunity by amplifying the biological effects of radiotherapy.

The interactions between nanomaterials and the immune system are multifaceted. Nanomaterials bolster immune activity by several mechanisms, including delivering immunoadjuvants to stimulate DC maturation, increasing TAA release, and modifying the TME to promote T-cell infiltration.[11] However, the innate and adaptive immune systems have evolved mechanisms to recognize and remove 'non-self' entities including nanomaterials. Circulating and tumor-resident macrophages can efficiently phagocytose nanomaterials to limit therapeutic benefit. The complex give-and-take between nanomaterials and the immune system presents multiple opportunities for improvement. For example, coating nanoparticles with CD47, a protein which evades phagocytosis, leads to reduced nanoparticle clearance rates.[12]

Delivery of therapies and circulating cells into tumors is limited by transport barriers including blood flow, extravasation, and interstitial diffusion. Radiotherapy may promote anti-tumor immunity by modifying these physiologic transport barriers.[13,14] A comprehensive understanding is required at the molecular, cellular, and tissue levels to delineate how MNPs dynamically modify the TME and the resulting biological response, including infiltration and efflux of immune cells. The extension from these efforts would focus on developing nanomaterials that modify the TME to optimize activation of antitumor immune pathways.

An important consideration when combining radiotherapy and immunotherapy is the potential for immune-related toxicity affecting multiple organ systems. While studies have shown combined radiotherapy and immunotherapy to be well-tolerated, others have reported increased toxicity rates. [15] Many nanomaterial-based strategies have focused on improving therapeutic efficacy, but there may be beneficial opportunities for nanomaterials to reduce immune-related systemic toxicity.

\section{Advances in science and technology to meet challenges}

Increasing immunological cell death with nanomaterials 
MNPs generate secondary electrons to amplify the radiation dose within tumors. Gold nanoparticles are established as radiotherapy enhancers via increased secondary electron production and reactive oxygen species (ROS) formation.[10] Investigators have established that gold nanoparticles can serve as radiosensitizers for megavoltage (MV) energies commonly used in the clinic.[16] Future work will clarify whether gold nanoparticles and radiotherapy can modulate anti-tumor immunity Hafnium oxide nanoparticles are currently in clinical trials for head and neck cancer, soft tissue sarcoma, prostate cancer, and liver cancer. Initial data suggest that hafnium oxide nanoparticles combined with radiotherapy may increase ICD, T-cell infiltration, activation of adaptive immunity, and cytokine signaling compared to radiotherapy alone.[17] While intriguing, more data is needed through prospective clinical trials to determine the immunomodulatory properties of hafnium oxide nanoparticles. Additional nanomaterials including gadolinium, platinum, and cerium oxide have been explored as radiotherapy enhancers.[18] Because of their shared mechanisms for enhancing radiotherapy, approaches combining MNPs with chemical oxygen mimetics such as nimorazole or misonidazole may be beneficial for local control of treatment-refractory hypoxic tumors.

Immune-Nanomaterial interactions in the tumor microenvironment

Nanomaterials designed to modulate the TME are engineered to have pharmacokinetic (PK) and biodistribution properties that permit accumulation in the TME. Numerous studies have found that for particles of similar charge and hydrophilicity, the principal systemic clearance mechanisms are renal and hepatobiliary excretion for smaller and larger particles, respectively.[19] An additional factor is the interaction of nanomaterials with circulating and tissue-resident macrophages. CD47-coated nanoparticles showed reduced rates of phagocytosis by activated $\mathrm{M} 1$ macrophages, demonstrating that surface modifications of nanomaterials can alter the interaction between nanomaterials and immune cells.[12] Incorporating immunoadjuvants into nanomaterials represents a promising approach to modify the TME. While radiotherapy can be transiently immunostimulatory, opposing immunosuppressive factors in the TME may negate this effect.[3] Nanomaterials localized to the TME that deliver a sustained release of 
immunostimulatory molecules may be beneficial for activating anti-tumor immunity. Research aimed at deciphering the multiscale interactions between nanomaterials, the TME, and immune system will be informative toward these efforts. Advanced imaging techniques such as intravital microscopy, genetically-engineered mouse models (GEMMs), and fluorescently-labeled cells and nanomaterials may be combined in innovative ways to reveal dynamic interactions in tumors.

Nanomaterials to reduce toxicity of radiotherapy and immunotherapy

Using nanomaterials to deliver immunotherapy to the TME and surrounding lymphatics may offer an approach for minimizing toxicities of systemic immunotherapy. A recent study investigated this approach in a preclinical model in which tumors received radiotherapy with intratumorally-administered anti-CD40 antibody in an emulsified formulation that permitted sustained local release.[20] Maximizing the intratumoral concentration of nanomaterials to reduce/systemic toxicity can be achieved by at least several mechanisms. Direct intratumoral injection of nanomaterials is being explored in preclinical and clinical studies. Delivery of molecules using nanoparticle carriers can also increase local concentrations of immunotherapies while reducing systemic toxicities. Efforts to target nanomaterials woúld include cataloging secreted proteins in the TME and those upregulated following radiotherapy to identify potential targeting ligands. Inter- and intra-tumor variability are relevant factors, as tumor sequencing has revealed considerable genetic and molecular heterogeneity between tumors.

\section{Concluding Remarks}

While immunotherapy has transformed modern oncology, many patients fail to achieve long-term, durable responses. Efforts are underway to bolster responses by combining radiotherapy with immunotherapy. Given the role of MNPs as radiotherapy enhancers, it follows that combinations of MNPs, radiotherapy, and immunotherapy may improve local and distant tumor control. We have highlighted several challenges in this emerging field. Can MNPs increase ICD following radiotherapy? Can MNPs evade macrophages and other clearance mechanisms in vivo? Can MNPs reduce the toxicity of systemic 
immunotherapy? The multiscale complexity of this work requires collaborations between biologists, chemists, engineers, and clinicians. Based on early results, there is cause for cautious optimism that nanomaterials-based solutions will maximize immunotherapy and radiotherapy responses in cancer patients.

\section{References}

[1] Seiwert TY, Burtness B, Mehra R, Weiss J, Berger R, Eder JP, et al. Safety and clinical activity of pembrolizumab for treatment of recurrent or metastatic squamous cell carcinoma of the head and neck (KEYNOTE-012): an open-label, multicentre, phase 1b trial. Lancet Oncol 2016;17:956-65. doi:10.1016/S14702045(16)30066-3.

[2] Topalian SL, Hodi FS, Brahmer JR, Gettinger SN, Smith DC, McDermott DF, et al. Safety, activity, and immune correlates of anti-PD-1 antibody in cancer. N Engl J Med 2012;366:2443-54. doi:10.1056/NEJMoa1200690.

[3] Weichselbaum RR, Liang H, Deng L, Fu YX. Radiotherapy and immunotherapy: A beneficial liaison? Nat Rev Clin Oncol/2017;14:365-79.

doi:10.1038/nrclinonc.2016.211.

[4] Antonia SJ, Villegas A, Daniel D, Vicente D, Murakami S, Hui R, et al. Durvalumab after Chemoradiotherapy in Stage III Non-Small-Cell Lung Cancer. N Engl J Med 2017;377:1919-29. doi:10.1056/NEJMoa1709937.

[5] Palma DA, Olson R, Harrow S, Gaede S, Louie A V., Haasbeek C, et al. Stereotactic ablative radiotherapy versus standard of care palliative treatment in patients with oligometastatic cancers (SABR-COMET): a randomised, phase 2, open-label trial. Lancet 2019;393:2051-8. doi:10.1016/S0140-6736(18)32487-5.

[6] Theelen WSME, Peulen HMU, Lalezari F, van der Noort V, de Vries JF, Aerts JGJ $\checkmark$, et al. Effect of Pembrolizumab After Stereotactic Body Radiotherapy vs Pembrolizumab Alone on Tumor Response in Patients With Advanced Non-Small Cell Lung Cancer: Results of the PEMBRO-RT Phase 2 Randomized Clinical 
Trial. JAMA Oncol 2019. doi:10.1001/jamaoncol.2019.1478.

[7] Postow MA, Callahan MK, Barker CA, Yamada Y, Yuan J, Kitano S, et al. Immunologic correlates of the abscopal effect in a patient with melanoma. N Engl J Med 2012;366:925-31. doi:10.1056/NEJMoa1112824.

[8] Vanpouille-Box C, Alard A, Aryankalayil MJ, Sarfraz Y, Diamond JM, Schneider $\mathrm{RJ}$, et al. DNA exonuclease Trex1 regulates radiotherapy-induced tumour immunogenicity. Nat Commun 2017;8:15618. doi:10.1038/ncomms15618.

[9] Twyman-Saint Victor C, Rech AJ, Maity A, Rengan R, Pauken KE, Stelekati E, et al. Radiation and dual checkpoint blockade activate non-redundant immune mechanisms in cancer. Nature 2015;520:373-7. doi:10.1038/nature14292.

[10] Hainfeld JF, Slatkin DN, Smilowitz HM. The use of gold nanoparticles to enhance radiotherapy in mice. Phys Med Biol 2004;49:N309-15.

[11] Min Y, Roche KC, Tian S, Eblan MJ, McKinnon KP, Caster JM, et al. Antigencapturing nanoparticles improve the abscopal effect and cancer immunotherapy. Nat Nanotechnol 2017;12:877-82. doi:10.1038/nnano.2017.113.

[12] Qie Y, Yuan H, von Roemeling CA, Chen Y, Liu X, Shih KD, et al. Surface modification of nanoparticles enables selective evasion of phagocytic clearance by distinct macrophage phenotypes. Sci Rep 2016;6:26269.

doi:10.1038/srep26269.

[13] Hellevik T, Martinez-Zubiaurre I. Radiotherapy and the tumor stroma: the importance of dose and fractionation. Front Oncol 2014;4:1. doi:10.3389/fonc.2014.00001.

[14] Miller MA, Chandra R, Cuccarese MF, Pfirschke C, Engblom C, Stapleton S, et al. Radiation therapy primes tumors for nanotherapeutic delivery via macrophagemediated vascular bursts. Sci Transl Med 2017;9:eaal0225. doi:10.1126/scitransImed.aal0225.

[15] Hwang WL, Niemierko A, Hwang KL, Hubbeling H, Schapira E, Gainor JF, et al. Clinical Outcomes in Patients With Metastatic Lung Cancer Treated With PD- 
1/PD-L1 Inhibitors and Thoracic Radiotherapy. JAMA Oncol 2018;4:253-5. doi:10.1001/jamaoncol.2017.3808.

[16] Wolfe T, Chatterjee D, Lee J, Grant JD, Bhattarai S, Tailor R, et al. Targeted gold nanoparticles enhance sensitization of prostate tumors to megavoltage radiation therapy in vivo. Nanomedicine Nanotechnology, Biol Med 2015;11:1277-83. doi:10.1016/j.nano.2014.12.016.

[17] Galon J, Laé M, Papai Z, Rochaix P, Mangel LC, Mlecnik B, et al. Specific adaptive immune pattern induced by NBTXR3 exposed to radiation therapy in soft tissue sarcoma (STS) patients. J Clin Oncol 2017;35:e14615-e14615. doi:10.1200/JCO.2017.35.15_suppl.e14615.

[18] Kuncic Z, Lacombe S. Nanoparticle radio-enhancement: principles, progress and application to cancer treatment. Phys Med Biol 2018;63:02TR01. doi:10.1088/1361-6560/aa99ce.

[19] Lin Z, Monteiro-Riviere NA, Riviere JE. Pharmacokinetics of metallic nanoparticles. Wiley Interdiscip Rev Nanomed Nanobiotechnol 2015;7:189-217. doi:10.1002/wnan.1304.

[20] Hao Y, Yasmin-Karim S, Moreau M, Sinha N, Sajo E, Ngwa W. Enhancing radiotherapy for lung cancer using immunoadjuvants delivered in situ from new design radiotherapy biomaterials: a preclinical study. Phys Med Biol 2016;61:N697-707. doi:10.1088/1361-6560/61/24/N697. 


\title{
Concluding Remarks:
}

\author{
Jan Schuemann ${ }^{1}$, and Sunil Krishnan ${ }^{2}$ \\ MA 02114, USA \\ 2 Department of Radiation Oncology, Mayo Clinic Florida, Jacksonville, FL
}

1 Department of Radiation Oncology, Massachusetts General Hospital \& Harvard Medical School, Boston,

This special collection of mini-articles on metal nanoparticles in radiation therapy provides a series of commentaries on the state-of-the-science and where the field in headed in the future, both in the laboratory and in the clinic. Drawing on the expertise of a non-complete selection of thought leaders in the field, this collection charts out a roadmap for future research activities. Each commentary outlines lessons learned on the path to where the field is today, highlights perceived challenges as the field advances towards the future, and finally discusses scientific and technological advances that might help overcome hurdles in the future. Although this is a compilation of several commentaries, the terseness of each commentary (mandated by the journal and us) makes for easy reading of multiple viewpoints from multiple perspectives and disciplines, all coalesced under one umbrella. We believe such a compilation of opinion pieces is especially interesting for an uninitiated reader who is looking for a high-level overview in broad brushstrokes. More in-depth information is readily available in articles referenced by the authors, many of which are co-authored by the same experts who wrote the commentary. For more advanced readers, these articles offer a summary of current challenges and an outlook of where we envision the field is headed.

A theme that emerges across all commentaries is that the science of fabricating, characterizing, decorating, imaging, and modeling of metal nanoparticles is mature and highly sophisticated. Nanoparticles are now used for a large variety of applications, most notably for functional imaging that has already advanced into the clinic. In the theranostic or radiation dose enhancing realm, the understanding of the underlying physical, chemical and biological consequences of interactions between these nanoparticles and ionizing radiation is evolving rapidly. Preclinical data across research teams are exciting and consistent in their ability to enhance radiation therapy effectiveness via physical and biological radiation sensitization. Against this background of palpable excitement are broad efforts to translate these technological advances to clinical reality after addressing safety, biocompatibility and scale-up issues. These clinical adoption strategies may span a variety of metallic nanoparticle constructs, a spectrum of targets within the tumor microenvironment, and combinations with other therapies including chemotherapy or immunotherapy. The enormous progress made in our understanding of fundamental nanoparticle-radiation interactions is especially meaningful because it has required the concerted efforts of scientists in disparate disciplines working individually or, more often, in unison to achieve these goals. While challenging questions remain regarding the best way to synergize nanoparticle use with radiation therapy without collateral toxicity, the overall therapeutic approach remains highly promising. As can be seen from the articles, all of the authors share the vision of advancing the outcome of radiation therapy using various constructs of nanoparticles by providing better imaging or therapeutic effects or a combination of both. Widespread clinical translation is long overdue. We hope this collection of research reflections and anticipated future directions will play a small role in making our vision of routine clinical use of metallic nanoparticles to enhance therapy a reality. 